\title{
CONSTRAINING THE EXOZODIACAL LUMINOSITY FUNCTION OF MAIN-SEQUENCE STARS: COMPLETE RESULTS FROM THE KECK NULLER MID-INFRARED SURVEYS
}

\author{
B. Mennesson ${ }^{1}$, R. Millan-Gabet ${ }^{2}$, E. Serabyn ${ }^{1}$, M. M. Colavita ${ }^{1}$, O. Absil $^{3}$, G. Bryden $^{1}$, M. Wyatt $^{4}$, W. Danchi ${ }^{5}$,

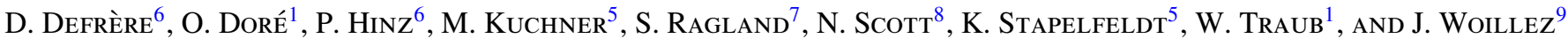 \\ ${ }^{1}$ Jet Propulsion Laboratory, California Institute of Technology, 4800 Oak Grove Drive, Pasadena, CA 91109-8099, USA; Bertrand.Mennesson@jpl.nasa.gov \\ 2 NASA Exoplanet Science Center, California Institute of Technology, 770 South Wilson Avenue, Pasadena, CA 91125, USA \\ ${ }^{3}$ Département d'Astrophysique, Géophysique et Océanographie, Université de Liège, 17 Allée du Six Août, 4000 Liège, Belgium \\ ${ }^{4}$ Institute of Astronomy, University of Cambridge, Madingley Road, Cambridge CB3 0HA, UK \\ 5 NASA Goddard Space Flight Center, Exoplanets and Stellar Astrophysics Laboratory, Code 667, Greenbelt, MD 20771, USA \\ ${ }^{6}$ Steward Observatory, Department of Astronomy, University of Arizona, 933 N. Cherry Avenue, Tucson, AZ 85721, USA \\ ${ }^{7}$ Keck Observatory, 65-1120 Mamalahoa Highway, Kamuela, HI 96743, USA \\ ${ }^{8}$ Center for High Angular Resolution Astronomy, Georgia State University, Mount Wilson, CA 91023, USA \\ ${ }^{9}$ European Southern Observatory, Karl-Schwarzschild-Strasse 2, D-85748 Garching bei Munchen, Germany \\ Received 2014 July 5; accepted 2014 October 1; published 2014 December 8
}

\begin{abstract}
Forty-seven nearby main-sequence stars were surveyed with the Keck Interferometer mid-infrared Nulling instrument (KIN) between 2008 and 2011, searching for faint resolved emission from exozodiacal dust. Observations of a subset of the sample have already been reported, focusing essentially on stars with no previously known dust. Here we extend this previous analysis to the whole KIN sample, including 22 more stars with known near- and/or far-infrared excesses. In addition to an analysis similar to that of the first paper of this series, which was restricted to the $8-9 \mu \mathrm{m}$ spectral region, we present measurements obtained in all 10 spectral channels covering the $8-13 \mu \mathrm{m}$ instrumental bandwidth. Based on the 8-9 $\mu \mathrm{m}$ data alone, which provide the highest signal-to-noise measurements, only one star shows a large excess imputable to dust emission ( $\eta$ Crv), while four more show a significant $(>3 \sigma)$ excess: $\beta$ Leo, $\beta \mathrm{UMa}, \zeta$ Lep, and $\gamma$ Oph. Overall, excesses detected by KIN are more frequent around A-type stars than later spectral types. A statistical analysis of the measurements further indicates that stars with known far-infrared $(\lambda \geqslant 70 \mu \mathrm{m})$ excesses have higher exozodiacal emission levels than stars with no previous indication of a cold outer disk. This statistical trend is observed regardless of spectral type and points to a dynamical connection between the inner (zodi-like) and outer (Kuiper-Belt-like) dust populations. The measured levels for such stars are clustering close to the KIN detection limit of a few hundred zodis and are indeed consistent with those expected from a population of dust that migrated in from the outer belt by Poynting-Robertson drag. Conversely, no significant midinfrared excess is found around sources with previously reported near-infrared resolved excesses, which typically have levels of the order of $1 \%$ over the photospheric flux. If dust emission is really at play in these near-infrared detections, the absence of a strong mid-infrared counterpart points to populations of very hot and small (submicron) grains piling up very close to the sublimation radius. For solar-type stars with no known infrared excess, likely to be the most relevant targets for a future exo-Earth direct imaging mission, we find that their median zodi level is $12 \pm 24$ zodis and lower than 60 (90) zodis with 95\% (99\%) confidence, if a lognormal zodi luminosity distribution is assumed.
\end{abstract}

Key words: circumstellar matter - infrared: stars - instrumentation: interferometers

Online-only material: color figures

\section{INTRODUCTION}

Debris disks found around main-sequence stars are the remnants of planetary formation. The outer colder parts of these disks, analogous to our solar system Kuiper-Edgeworth (hereafter Kuiper) Belt, were first detected via their midinfrared (MIR) or far-infrared (FIR) excess emission and then imaged at visible to submillimeter wavelengths. Structures and asymmetries in spatially resolved debris disks have been used to infer the presence of yet unseen planets. The power of this technique was recently demonstrated with the direct imaging of a massive planet at the inner edge of the warped extended dust disk previously detected around $\beta$ Pic (Lagrange at al. 2009; Macintosh et al. 2014). Conversely, very little is known about the hotter (>200 K) dust component of debris disks, concentrated in the inner few $\mathrm{AU}$ of the stellar environment where rocky planets may have formed, similar to the zodiacal dust found in the inner solar system, which originates from the tails of comets and from collisions between asteroids in the asteroid belt.
Indeed, only a few hot debris disks have been found by Spitzer around mature stars from excess emission at wavelengths of $24 \mu \mathrm{m}$ or shorter (Beichman et al. 2006a; Lawler et al. 2009), and only a few have been unambiguously resolved so far (e.g., Smith et al. 2009). This observational difficulty results from two main factors: the exozodiacal disks' small angular sizes, and their faintness relative to the host star. Indeed, while cold debris disks cause very significant excesses readily detectable at FIR wavelengths, exozodiacal material located in the inner few AU only contributes a small fraction of the stellar MIR flux. In order to reliably detect such tiny $(\simeq 1 \%)$ excess emission over that expected from the photosphere, direct imaging is required, with the ability to spatially resolve dust from the central star. In the visible, where dust is seen by means of scattered starlight, the contrast required is extremely high and only a space- or balloon-borne coronagraph could provide adequate performance. In the infrared, exozodiacal disks produce significant thermal emission and contribute a larger fraction of the stellar flux, making it the spectral range of choice for 
ground-based exozodiacal studies. With the spatial scales at play, typically 0.1 to a few AU, such direct infrared observations are best accomplished using long-baseline interferometry. This was the main goal of the Keck Interferometer Nuller (KIN), a long-baseline $(85 \mathrm{~m})$, high-contrast instrument operating between 8 and $13 \mu \mathrm{m}$, especially built to spatially resolve faint structures next to bright stars (Serabyn et al. 2012; Colavita et al. 2013). Exozodiacal observations were carried out with the KIN between 2008 and 2011 through three different Key Science programs (led by Serabyn, Hinz, and Kuchner, respectively) and one PI program (led by Mennesson). These studies targeted a total of 47 nearby main-sequence stars whose basic properties are listed in Table 1 . We already analyzed a subset of this sample (MillanGabet et al. 2011, hereafter Paper I) comprising 23 stars with no previously known dust and 2 with dust previously detected in the MIR and FIR: $\eta$ Crv (Beichman et al. 2006b; Chen et al. 2006; Smith et al. 2009) and $\gamma$ Oph (Su et al. 2008). This initial data analysis was restricted to a single spectral channel spanning 8-9 $\mu \mathrm{m}$ and revealed only one clear excess (around $\eta \mathrm{Crv}$ ) and two marginal ones (around $\gamma$ Oph and Altair). It also provided the best limits to date on $10 \mu \mathrm{m}$ exozodi levels for a sample of nearby main-sequence stars, with a typical measurement uncertainty $(1 \sigma)$ of 150 times the solar zodiacal light emission, or 150 "zodis." For all stars in our sample, we define the unit zodi case as a dust cloud with the same optical depth at $1 \mathrm{AU}$ and the same radial density profile as measured in the solar system (Kelsall et al. 1998), but with an inner dust radius and radial temperature profile that scale with stellar luminosity (see Section 3.5 for further details).

In this context, our present goals are to (1) perform a final calibration of the complete KIN sample of 47 stars using a consistent set of rules for data vetting, calibrator diameters, and uncertainties; (2) compute all individual star excess measurements over the full $8-13 \mu \mathrm{m}$ spectral range, divided into 10 spectral channels; (3) assess possible correlations between basic stellar properties and measured MIR excesses; and (4) derive conclusions on the prevalence of high levels of exozodi emission for nearby Sun-like stars. As the more sensitive Large Binocular Telescope Interferometer (LBTI) exozodi survey is just starting, it is also timely to draw a final set of conclusions from the KIN surveys to help refine the LBTI stellar sample selection (Weinberger et al. 2014).

\section{CALIBRATION AND DATA VETTING}

The KIN data acquisition principle and reduction technique are fully described in past publications (Colavita et al. 2009; Millan-Gabet et al. 2011; Serabyn et al. 2012) and will not be discussed in detail here. Similar to regular interferometric observations, the correction of instrumental effects is based on interspersed nulling observations of science targets and nearby calibrator stars. These calibrators have well-known diameters and are used to derive accurate estimates of the instrumental leakage at the time of science target observations.

\subsection{Calibrators}

In order to minimize systematic errors in the calibration, calibrator stars were chosen close to the science targets and with similar MIR fluxes. As a result, these calibrator stars were typically giants. In order to most accurately predict their angular diameters, we retained only the observations of giant calibrators with spectral types $\mathrm{G}, \mathrm{K}$, and early $\mathrm{M}$. We also made a thorough check through the literature and rejected any cali- brator with a reference to possible binarity or variability. As stated in Paper I, these different criteria minimize the possibility of infrared emission above photospheric levels (Cohen et al. 1999). ${ }^{10}$ The calibrators' limb-darkened (LD) diameters were computed adopting the following set of rules:

1. If a calibrator was listed in the Bordé et al. (2002) or Merand et al. (2006) catalogs of interferometric calibrators, we adopted its estimated LD diameter, with a relative error bar of $\pm 5 \%$. This error is conservatively increased in comparison to the catalog's quoted uncertainties (typically $1.5 \%$ ), reflecting the typical offset measured with respect to long-baseline interferometric measurements when available.

2. Otherwise, we used surface brightness relations, specifically the LD diameter versus $\left(V_{0}-K_{0}\right)$ relation, where $V_{0}$ and $K_{0}$ are the measured $V$ and $K$ magnitudes corrected for interstellar extinction. We assumed the extinction to be isotropic, i.e., just a function of stellar distance. Because common surface brightness relations are valid over specific ranges of color differences, we then considered two subcases:

(a) If the derived $V_{0}-K_{0}$ was between -0.1 and 3.6, we used the surface brightness relationship established by Di Benedetto (2005, Equations (2) and (4)). We then applied an LD diameter error bar of $\pm 6 \%$ if the $K$ magnitude came from Two Micron Sky Survey Infrared catalog (TMSS; Neugebauer \& Leighton 1969) or from JP11 (IRAS catalog; Gezari et al. 1993) measurements. We used an error bar of $\pm 10 \%$ if the $K$ magnitude came from the Two Micron All Sky Survey (2MASS), whose measurements are saturated and fairly inaccurate for our bright calibrators.

(b) If the derived $V_{0}-K_{0}$ was between 3.6 and 7 or between -1.1 and -0.1 , we used the surface brightness relationship established by Bonneau et al. (2006, Equations (9) and (10) and Table 2 of that paper), in the case of bright objects $(V<10, K<5)$. We applied an error bar of $\pm 7 \%$ if the $K$ magnitude came from TMSS or JP11 measurements. We used an error bar of $\pm 10 \%$ if the $K$ magnitude came from 2 MASS.

The error bars quoted above $(6 \%, 7 \%$, or $10 \%)$ were derived by comparing the derived surface brightness LD diameters with those estimated by Bordé et al. (2002) and Merand et al. (2006) for an ensemble of 100 calibrators (including those used in this work), assuming that the Bordé and Merand values were "the correct ones." It is worth noting that calibrator diameters were estimated in a different way in Paper I, which used Keck Interferometer $K$-band measurements acquired at the same time as the MIR null measurements. The present calibration strategy leads to slightly revised null values for the 25 science targets already discussed in Paper I. However, all changes are within the calibrated null error bars of a few times $10^{-3}$, with the exception of Altair (see Section 5.2).

\subsection{Data Vetting}

For each science target, we only retained null data sequences (or "scans") based on the following criteria: at least one calibrator observation is available within $1 \mathrm{hr}$, and the percentage

\footnotetext{
10 If there was any significant $N$-band excess emission around the calibrators, this would bias the science target measurements toward lower or even negative null excess levels, which we do not observe.
} 
Table 1

Target Properties and Observing Log

\begin{tabular}{|c|c|c|c|c|c|c|c|c|c|c|c|c|c|c|}
\hline HD & Star & $\begin{array}{l}\text { Spectral } \\
\text { Type }\end{array}$ & $\begin{array}{l}\text { Object } \\
\text { Type }\end{array}$ & $L_{\mathrm{star}} / L_{\odot}$ & $R_{\mathrm{star}} / R_{\odot}$ & $\begin{array}{l}T_{\text {star }} \\
(\mathrm{K})\end{array}$ & $\begin{array}{l}F_{\text {star }} \\
(\mathrm{Jy})\end{array}$ & $\begin{array}{l}\text { LD Diam } \\
\text { (mas) }\end{array}$ & $\begin{array}{l}\text { IWA } \\
(\mathrm{AU})\end{array}$ & $\begin{array}{l}\text { OWA } \\
(\mathrm{AU})\end{array}$ & $\begin{array}{l}R_{300 \mathrm{~K}} \\
(\mathrm{AU})\end{array}$ & $\mathrm{Nc}$ & $\begin{array}{l}\text { Dates } \\
\text { (UT) }\end{array}$ & $\overline{\mathrm{Ns}}$ \\
\hline 432 & $\beta$ Cas & F2IV & ecold & 27.3 & 3.4 & 7079 & 15.3 & $2.027 \pm 0.122$ & 0.10 & 3.4 & 5.2 & 2 & 2008 Aug 17 & 3 \\
\hline $\mathrm{N} / \mathrm{A}$ & $\eta$ Cas A & G3 & $\cdots$ & 1.3 & 1.0 & 6087 & 9.8 & $1.894 \pm 0.114$ & 0.04 & 1.2 & 1.1 & 2 & 2010 Sep 22 & 3 \\
\hline 9826 & $v$ And & F9V & $\cdots$ & 3.3 & 1.6 & 6120 & 3.9 & $1.021 \pm 0.060$ & 0.08 & 2.7 & 1.8 & 1 & 2008 Nov 12 & 2 \\
\hline 10476 & 107 Psc & $\mathrm{K} 1 \mathrm{~V}$ & $\cdots$ & 0.4 & 0.8 & 5180 & 2.6 & $1.067 \pm 0.064$ & 0.04 & 1.5 & 0.7 & 2 & 2008 Oct 13 & 2 \\
\hline 10700 & $\tau$ Ceti & G8V & eboth & 0.5 & 0.8 & 5344 & 11.8 & $2.015 \pm 0.023$ & 0.02 & 0.7 & 0.7 & 5 & $\begin{array}{l}2008 \text { Oct } 14 \\
2008 \text { Oct } 15\end{array}$ & $\begin{array}{l}4 \\
2\end{array}$ \\
\hline 13161 & $\beta$ Tri & A5III & bin3 & 71.0 & 4.4 & 8020 & 4.7 & $1.050 \pm 0.100$ & 0.23 & 7.8 & 8.4 & 2 & 2008 Aug 18 & 1 \\
\hline 13974 & $\delta$ Tri & G0V & bin3 & 1.1 & 1.0 & 5860 & 2.7 & $1.105 \pm 0.111$ & 0.07 & 2.2 & 1.0 & 2 & 2008 Nov 13 & 2 \\
\hline 16895 & $\theta$ Per & F7V & $\cdots$ & 2.2 & 1.2 & 6320 & 4.0 & $1.086 \pm 0.056$ & 0.07 & 2.2 & 1.5 & 3 & $\begin{array}{l}\text { 2009 Jan } 11 \\
\text { 2009 Jan } 12\end{array}$ & $\begin{array}{l}2 \\
1\end{array}$ \\
\hline 19373 & $\iota$ Per & F9V & $\cdots$ & 2.2 & 1.4 & 5890 & 4.7 & $1.086 \pm 0.056$ & 0.06 & 2.1 & 1.5 & 4 & 2009 Jan 11 & 1 \\
\hline 20630 & $\kappa-1 \mathrm{Cet}$ & G5V & $\cdots$ & 0.8 & 1.0 & 5620 & 2.7 & $0.895 \pm 0.070$ & 0.05 & 1.8 & 0.9 & 2 & 2009 Jan 10 & 1 \\
\hline 22049 & $\epsilon$ Eri & $\mathrm{K} 2 \mathrm{~V}$ & ecold & 0.3 & 0.7 & 5084 & 12.2 & $2.126 \pm 0.131$ & 0.02 & 0.6 & 0.5 & 3 & $\begin{array}{l}2008 \text { Oct } 13 \\
2008 \text { Oct } 14 \\
\text { 2009 Jan } 13\end{array}$ & $\begin{array}{l}2 \\
3 \\
1\end{array}$ \\
\hline 22484 & $10 \mathrm{Tau}$ & F8V & eboth & 3.0 & 1.6 & 5981 & 3.7 & $1.130 \pm 0.068$ & 0.08 & 2.8 & 1.7 & 3 & 2011 Feb 14 & 2 \\
\hline 30652 & 1 Ori & F6V & $\cdots$ & 2.6 & 1.3 & 6450 & 7.9 & $1.409 \pm 0.050$ & 0.05 & 1.6 & 1.6 & 3 & $\begin{array}{l}2008 \text { Feb } 17 \\
2008 \text { Feb } 18\end{array}$ & $\begin{array}{l}3 \\
1\end{array}$ \\
\hline 34411 & $\lambda$ Aur & G1IV-V & $\cdots$ & 1.7 & 1.3 & 5820 & 2.7 & $0.940 \pm 0.056$ & 0.08 & 2.5 & 1.3 & 2 & 2009 Jan 12 & 1 \\
\hline 38393 & $\gamma$ Lep & F6V & $\cdots$ & 2.3 & 1.2 & 6410 & 5.9 & $1.871 \pm 0.112$ & 0.05 & 1.8 & 1.5 & 3 & $\begin{array}{l}2009 \text { Jan } 10 \\
2009 \text { Jan } 13\end{array}$ & $\begin{array}{l}1 \\
1\end{array}$ \\
\hline 38678 & $\zeta$ Lep & A2IV-V & ecold & 14.0 & 1.5 & 9772 & 2.6 & $0.670 \pm 0.140$ & 0.13 & 4.3 & 3.7 & 5 & $\begin{array}{l}\text { 2008 Nov } 12 \\
\text { 2009 Jan } 9 \\
\text { 2009 Jan } 12 \\
\text { 2009 Jan } 13 \\
\text { 2011 Feb } 14\end{array}$ & $\begin{array}{l}2 \\
1 \\
1 \\
1 \\
1\end{array}$ \\
\hline 39587 & $\chi-1$ Ori & G0V & bin 1 & 1.0 & 1.0 & 5930 & 3.6 & $1.124 \pm 0.057$ & 0.05 & 1.7 & 1.0 & 4 & $\begin{array}{l}2009 \text { Jan } 10 \\
2009 \text { Jan } 13\end{array}$ & $\begin{array}{l}2 \\
2\end{array}$ \\
\hline 40136 & $\eta$ Lep & $\mathrm{F} 2 \mathrm{~V}$ & eboth & 4.6 & 1.5 & 6900 & 3.7 & $0.987 \pm 0.059$ & 0.09 & 3.0 & 2.1 & 4 & $\begin{array}{l}\text { 2009 Jan } 9 \\
\text { 2009 Jan } 10 \\
\text { 2009 Jan } 13\end{array}$ & $\begin{array}{l}3 \\
1 \\
1\end{array}$ \\
\hline 56537 & $\lambda \mathrm{Gem}$ & A3V & ehot & 27.8 & 2.0 & 9380 & 2.6 & $0.644 \pm 0.006$ & 0.19 & 6.2 & 5.3 & 2 & 2010 Sep 22 & 2 \\
\hline 88230 & NSV 4765 & $\mathrm{~K} 8 \mathrm{~V}$ & $\cdots$ & 0.1 & 0.8 & 3920 & 3.1 & $1.238 \pm 0.054$ & 0.03 & 1.0 & 0.4 & 2 & 2009 Jan 10 & 1 \\
\hline 95128 & $47 \mathrm{UMa}$ & G1V & $\cdots$ & 1.6 & 1.2 & 5860 & 2.0 & $0.774 \pm 0.073$ & 0.08 & 2.8 & 1.2 & 4 & $\begin{array}{l}2009 \operatorname{Jan} 10 \\
2009 \operatorname{Jan} 11\end{array}$ & $\begin{array}{l}1 \\
1\end{array}$ \\
\hline 95418 & $\beta \mathrm{Uma}$ & $\mathrm{A} 1 \mathrm{~V}$ & ecold & 63.0 & 3.0 & 9377 & 6.0 & $1.078 \pm 0.065$ & 0.15 & 4.9 & 7.9 & 2 & $\begin{array}{l}2008 \text { May } 26 \\
2008 \text { May } 27\end{array}$ & $\begin{array}{l}2 \\
2\end{array}$ \\
\hline 95735 & HIP 54035 & $\mathrm{M} 2 \mathrm{~V}$ & $\cdots$ & 0.025 & 0.3 & 3730 & 3.2 & $1.439 \pm 0.050$ & 0.02 & 0.5 & 0.1 & 1 & $\begin{array}{l}2008 \text { Apr } 14 \\
\text { 2009 Jan } 10\end{array}$ & $\begin{array}{l}2 \\
1\end{array}$ \\
\hline 102647 & $\beta$ Leo & A3V & eboth & 15.0 & 1.7 & 8500 & 9.1 & $1.339 \pm 0.087$ & 0.07 & 2.2 & 3.9 & 2 & $\begin{array}{l}2008 \text { Feb } 18 \\
2008 \text { Apr } 16 \\
\text { 2009 Jan } 11\end{array}$ & $\begin{array}{l}2 \\
3 \\
1\end{array}$ \\
\hline 102870 & $\beta$ Vir & F9V & $\cdots$ & 3.4 & 1.7 & 6080 & 6.6 & $1.431 \pm 0.086$ & 0.07 & 2.2 & 1.9 & 3 & $\begin{array}{l}2008 \text { Feb } 17 \\
2008 \text { Feb } 18\end{array}$ & $\begin{array}{l}2 \\
4\end{array}$ \\
\hline 106591 & $\delta$ Uma & A3V & ecold & 14.0 & 1.4 & 9480 & 3.0 & $0.823 \pm 0.049$ & 0.15 & 4.9 & 3.7 & 4 & $\begin{array}{l}2008 \text { Apr } 16 \\
\text { 2009 Jan } 10\end{array}$ & $\begin{array}{l}3 \\
1\end{array}$ \\
\hline 109085 & $\eta \mathrm{Crv}$ & $\mathrm{F} 2 \mathrm{~V}$ & ecold & 4.7 & 1.5 & 6870 & 2.5 & $0.833 \pm 0.050$ & 0.11 & 3.6 & 2.2 & 5 & $\begin{array}{l}2008 \text { Apr } 17 \\
2008 \text { May } 24\end{array}$ & $\begin{array}{l}1 \\
3\end{array}$ \\
\hline 114710 & $\beta \mathrm{Com}$ & G0V & $\cdots$ & 1.3 & 1.1 & 5960 & 3.8 & $1.071 \pm 0.058$ & 0.05 & 1.8 & 1.2 & 3 & 2008 Feb 16 & 3 \\
\hline 115617 & 61 Vir & G7V & ecold & 0.9 & 1.0 & 5577 & 3.2 & $1.164 \pm 0.116$ & 0.05 & 1.7 & 0.9 & 3 & 2009 Jan 12 & 3 \\
\hline 117176 & 70 Vir & G5V & ecold & 3.1 & 1.9 & 5545 & 2.8 & $0.953 \pm 0.062$ & 0.11 & 3.6 & 1.8 & 5 & $\begin{array}{l}\text { 2008 Apr } 15 \\
\text { 2008 Apr } 17 \\
\text { 2009 Jan } 13\end{array}$ & $\begin{array}{l}1 \\
3 \\
2\end{array}$ \\
\hline 120136 & $\tau$ Boo & F6IV & bin 1 & 3.0 & 1.4 & 6370 & 2.5 & $0.864 \pm 0.066$ & 0.09 & 3.1 & 1.7 & 2 & $\begin{array}{l}2008 \text { May } 25 \\
2008 \text { May } 27\end{array}$ & $\begin{array}{l}3 \\
2\end{array}$ \\
\hline 131977 & KX Lib & $\mathrm{K} 4 \mathrm{~V}$ & $\cdots$ & 0.3 & 0.8 & 4570 & 3.1 & $1.490 \pm 0.089$ & 0.04 & 1.2 & 0.5 & 2 & 2008 May 26 & 2 \\
\hline 142091 & $\kappa \mathrm{Crb}$ & K1IV & eboth & 12.9 & 5.0 & 4877 & 5.5 & $1.550 \pm 0.093$ & 0.19 & 6.2 & 3.6 & 1 & 2011 Jun 25 & 2 \\
\hline 142860 & $\gamma \mathrm{Ser}$ & F6IV & $\cdots$ & 2.7 & 1.4 & 6370 & 4.7 & $1.161 \pm 0.055$ & 0.07 & 2.2 & 1.7 & 2 & $\begin{array}{l}2008 \text { Apr } 16 \\
2008 \text { Apr } 17\end{array}$ & $\begin{array}{l}3 \\
2\end{array}$ \\
\hline 161868 & $\gamma \mathrm{Oph}$ & A0V & ecold & 21.9 & 1.9 & 9030 & 1.8 & $0.630 \pm 0.063$ & 0.17 & 5.8 & 4.7 & 4 & $\begin{array}{l}2008 \text { Jul } 16 \\
2008 \text { Jul } 17\end{array}$ & $\begin{array}{l}3 \\
3\end{array}$ \\
\hline 165341 & $70 \mathrm{Oph}$ & $\mathrm{K} 0 \mathrm{~V}$ & $\cdots$ & 0.6 & 1.0 & 5140 & 9.8 & $2.037 \pm 0.122$ & 0.03 & 1.0 & 0.8 & 1 & $\begin{array}{l}2008 \text { Aug } 17 \\
2008 \text { Aug } 18\end{array}$ & $\begin{array}{l}2 \\
2\end{array}$ \\
\hline 172167 & Vega & $\mathrm{A} 0 \mathrm{~V}$ & eboth & 40.1 & 2.4 & 9602 & 53.9 & $3.306 \pm 0.030$ & 0.05 & 1.5 & 6.3 & 9 & $\begin{array}{l}2008 \text { May } 27 \\
2008 \text { Jul } 14\end{array}$ & $\begin{array}{l}4 \\
1\end{array}$ \\
\hline
\end{tabular}


Table 1

(Continued)

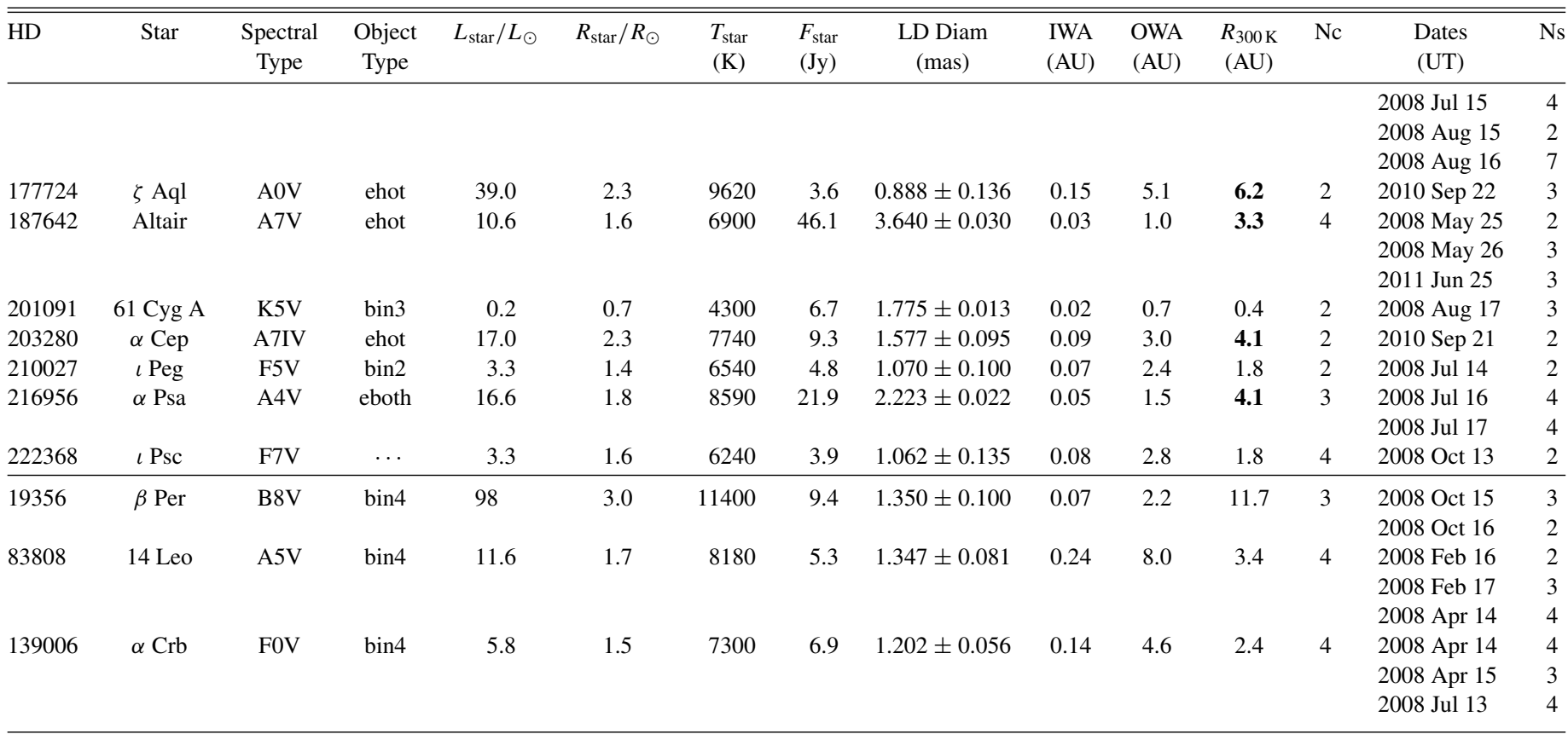

Notes. List of KIN targets sorted by increasing right ascension. Object type indicates any peculiarity about the target. "bin 1": binary system with companion outside of KIN FOV. "bin 2": binary system with companion of known properties within the KIN FOV and no obvious signature in the null measurements. "bin 3": binary system with companion of unknown brightness and no obvious signature within the KIN FOV. "bin4": binary system with companion inside the KIN FOV and some obvious signature in the null measurements. No zodiacal-level estimation was possible for these three "bin 4" stars, which are listed separately at the end of the table. "ecold": cold excess previously detected at MIR or FIR wavelengths. "ehot": hot excess previously detected in the NIR. "eboth": both cold and hot excess previously detected. $F_{\text {star }}: 10 \mu \mathrm{m}$ stellar flux in Jy. LD Diam: stellar LD diameter in mas. IWA/OWA: KIN inner and outer working angles (in AU). $R_{300 \mathrm{~K}}:$ minimum distance for $300 \mathrm{~K}$ dust (in $\mathrm{AU}$ ). $N_{c}$ : total number of calibrator stars used. $N_{s}$ : total number of null data sequences recorded.

of gated data (Colavita et al. 2009) retained for that scan is greater than $50 \%$, indicating reasonably good phase and angle tracking performance. We also checked that the nightly log sheets reported adequate observing conditions in terms of cloud cover and seeing, no instrumental configuration change or realignment between target and calibrator, no evidence for fast photometry dropouts, and no major issues with the atmospheric dispersion correctors (ADCs). Namely, we removed all scans with large sudden jumps or saturation (hard limit reach) of the ADC prism's position, indicating strongly variable or poor instrumental nulls.

\section{RESULTS}

\subsection{Excess Leak Curves}

As described in Paper I and in Serabyn et al. (2012), the quantity observed by the KIN may be understood by projecting the fringe pattern on the sky: what is measured is the astrophysical flux from the object's surface brightness transmitted through a four-beam nuller fringe pattern (see Appendix A for a complete description). For the work presented here, we measure and calibrate the transmitted flux (expected to be small) due to dust surrounding the central target stars. Thus, we refer to the basic observable as the flux leakage or simply the "leak." The amount of flux leakage not attributable to the finite size of the central stars is referred to as "excess leak," and by measuring it we can learn about the amounts of circumstellar dust present. The data reduction steps defined hereafter were applied to all 47 science targets and are identical for all 10 spectral channels covering the full $8-13 \mu \mathrm{m}$ bandwidth of the instrument. Additionally, the real-time archiver saves the signal summed over the two spectral channels between 8 and $9 \mu \mathrm{m}$, forming the so-called 8-9 $\mu \mathrm{m}$ null bin already used in Paper I, which is treated here as an "11th" channel.

1. For each individual stellar scan (typically 5 minutes long), we computed a raw leak (observed null depth) versus wavelength.

2. We then subtracted the instrumental leakage measured on nearby calibrators, which yields the target's calibrated leak versus wavelength.

3. Owing to their finite extension, all targets observed are slightly resolved by the KIN. In the case of a photosphere represented by an LD disk of diameter $\theta_{\mathrm{LD}}$ with a linear LD coefficient $u_{\lambda}$, the corresponding stellar leak is given by (Absil et al. 2006, 2011)

$$
L_{\text {star }}(\lambda)=\left(\frac{\pi B \theta_{\mathrm{LD}}}{4 \lambda}\right)^{2}\left(1-\frac{7 u_{\lambda}}{15}\right)\left(1-\frac{u_{\lambda}}{3}\right)^{-1}
$$

When working in the MIR, LD effects are generally small $(<0.1)$ for main-sequence stars. As a result, we generally assume $u_{\lambda}=0$ in the equation above. As long as the linear LD coefficient is smaller than 0.1 , this approximation translates into a stellar leak error of a few times $10^{-4}$ at most, which is completely negligible compared to our measurement uncertainty. The only two exceptions are Vega and Altair (see Section 5.2). Both are rapid rotators with significant-gravity induced - LD and large enough diameters that this effect must be taken into account when computing the stellar leak. For Vega, we adopted the model derived by Aufdenberg et al. (2006), which yields 

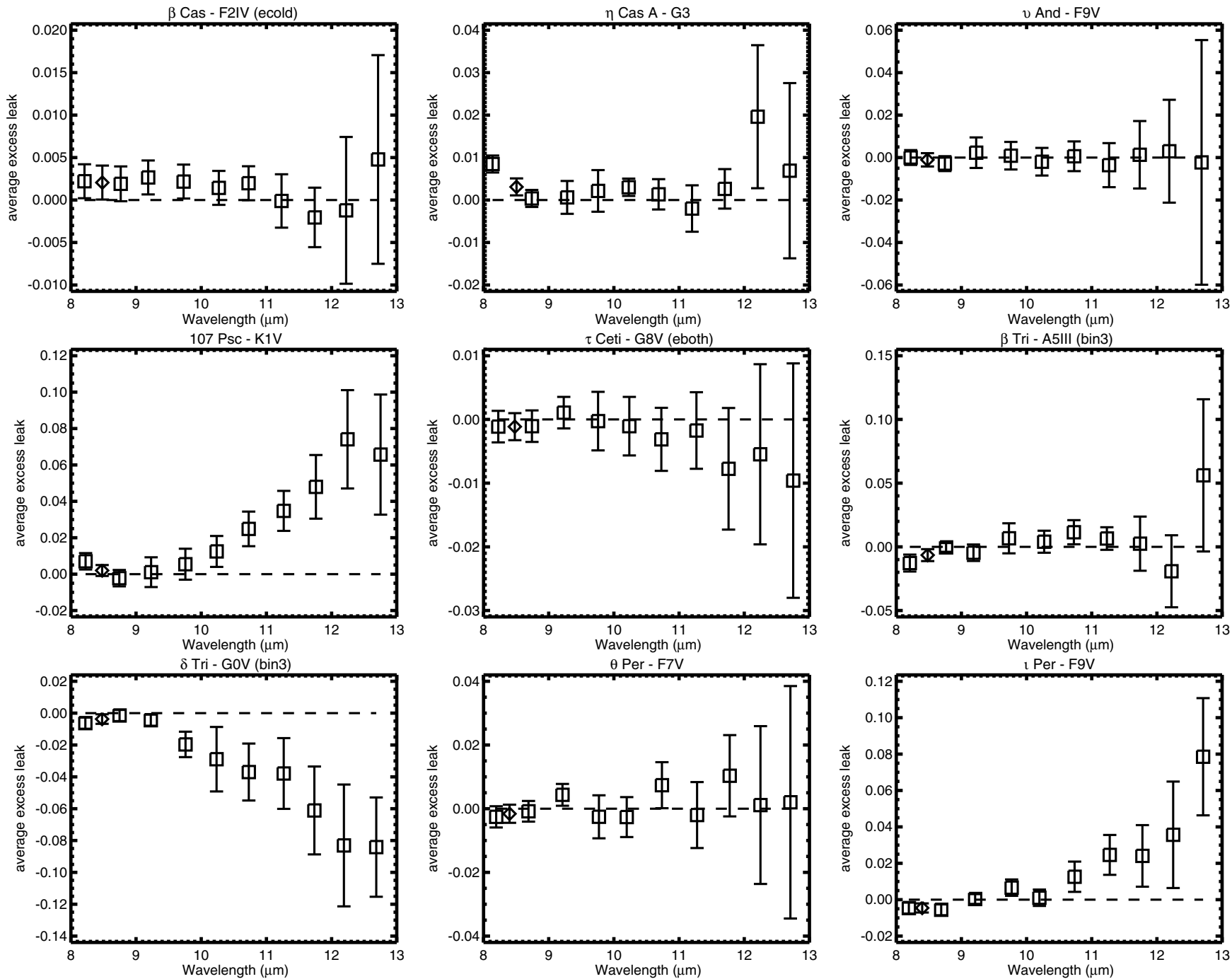

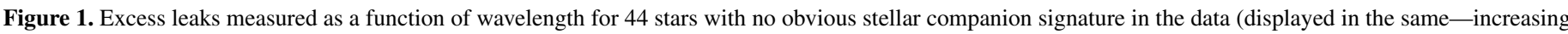

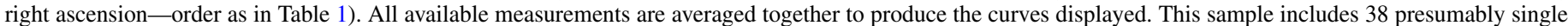

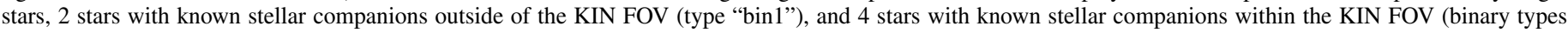

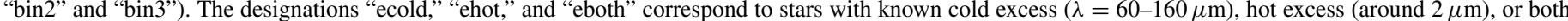

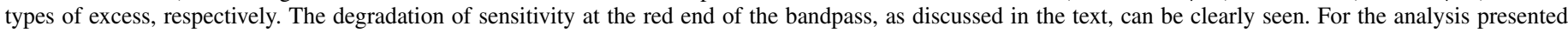

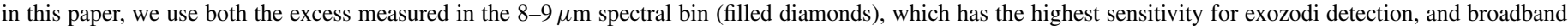
excess estimates based on the whole $8-13 \mu \mathrm{m}$ range.

$\theta_{\mathrm{LD}}=3.329 \pm 0.03$ mas and $u_{\lambda}=0.10$ between 8 and $13 \mu \mathrm{m}$. For Altair, we adopted for each KIN observation the position angle (P.A.) dependent diameter measured by Monnier et al. (2007) with the CHARA interferometer. At each wavelength and for each individual scan, the stellar leak is then subtracted from the calibrated leak defined above, yielding the measured excess leak versus wavelength.

4. A single measured excess leak versus wavelength curve is then computed per target, averaging all scans (typically between 1 and 4) obtained under a common set of instrumental conditions, which we refer to as an observational "cluster." " Say that a given cluster (index $j$ ) consists of a set of individual scans (index $i$ ) characterized at any single wavelength by an excess leak measurement $E_{i j}$ with uncertainty $\sigma_{i j}$. The average excess leak $E_{j}$ corresponding

\footnotetext{
${ }^{11}$ Unless a major optical realignment occurred during the observations (e.g. caused by a change of the static delay line position), there is only one observational cluster per night and per target.
}

to that cluster is computed as the weighted least-squares mean from individual scans:

$$
E_{j}=\frac{\sum_{i} E_{i j} / \sigma_{i j}^{2}}{\sum_{i} 1 / \sigma_{i j}^{2}}
$$

5. The uncertainty on the cluster excess leak is estimated by computing a statistical "internal" term $\sigma_{j}^{\text {int }}$, decreasing with the number of scans but still nonzero if all noisy measurements happen to be equal to

$$
\sigma_{j}^{\text {int }}=\frac{1}{\sqrt{\sum_{i} 1 / \sigma_{i j}^{2}}},
$$

and a second "external" error $\sigma_{j}^{\text {ext }}$ that reflects the scatter of individual measurements (weighted standard deviation),

$$
\sigma_{j}^{\mathrm{ext}}=\sqrt{\frac{\sum_{i}\left(E_{i j}-E_{j}\right)^{2} / \sigma_{i j}^{2}}{\sum_{i} 1 / \sigma_{i j}^{2}}} .
$$



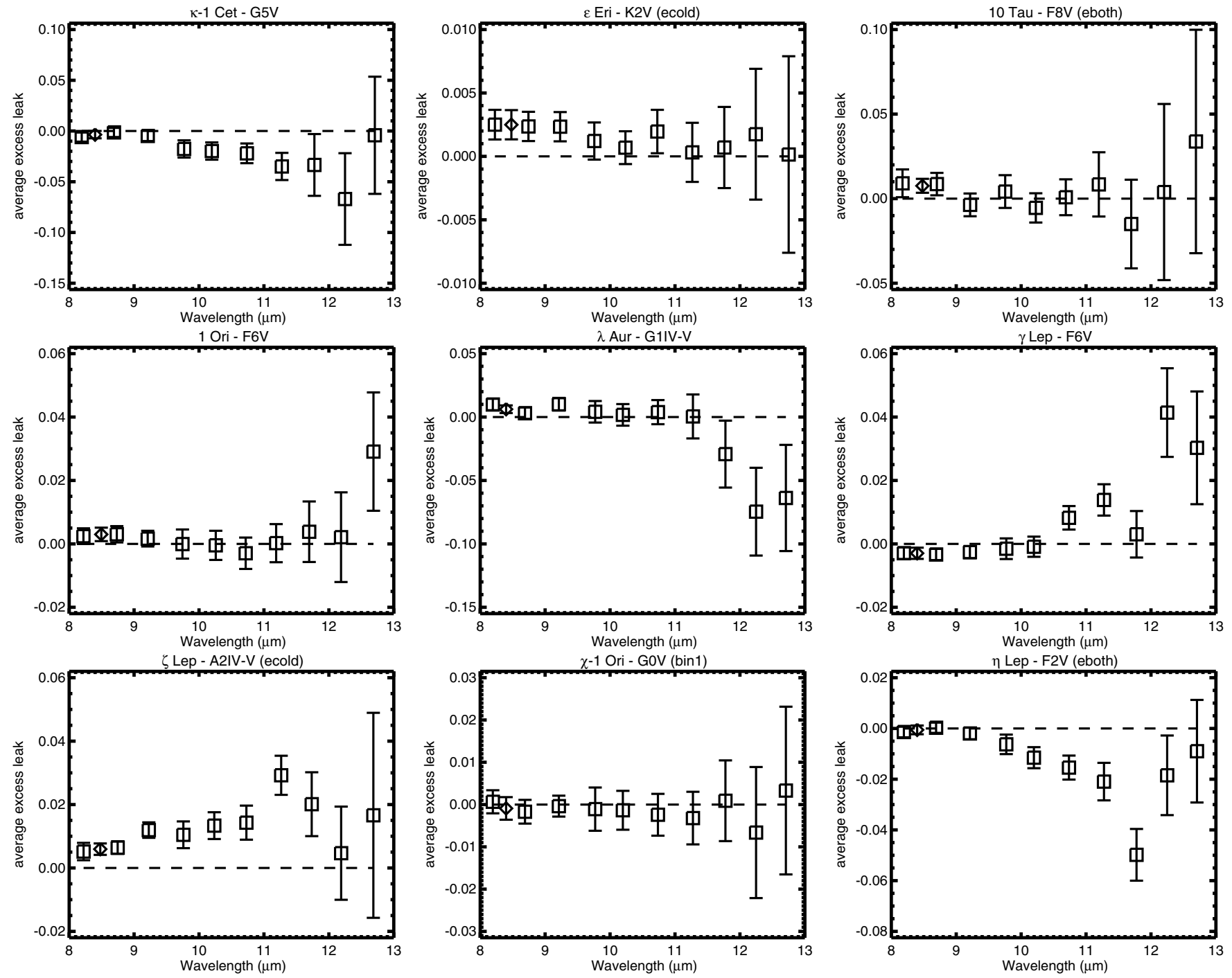

Figure 1. (Continued)

Both internal and external uncertainties are taken into account and summed in quadrature. The resulting uncertainty is then compared to the systematic error floor $\sigma_{\text {sys }}$ per cluster recommended in Table 2 of Colavita et al. (2009), which is a function of wavelength and stellar flux. Only the larger value is retained, and the final leak excess uncertainty per cluster is hence defined as

$$
\sigma_{j}=\max \left(\sqrt{\left(\sigma_{j}^{\text {int }}\right)^{2}+\left(\sigma_{j}^{\text {ext }}\right)^{2}}, \sigma_{\text {sys }}\right)
$$

6. Finally, in the case where several data clusters are available for a given target, a weighted mean excess leak is computed for each wavelength as

$$
E=\frac{\sum_{j} E_{j} / \sigma_{j}^{2}}{\sum_{j} 1 / \sigma_{j}^{2}},
$$

and its uncertainty is estimated as

$$
\sigma=\frac{1}{\sqrt{\sum_{j} 1 / \sigma_{j}^{2}}}
$$

As a summary, the final KIN data reduction products consist of stellar excess leak curves measured as a function of wavelength. As described in Paper I, in the usual case that the MIR circumstellar (exozodiacal) flux is small compared to the stellar photospheric flux, the excess leak $(E)$ is proportional to the MIR flux emitted by the exozodiacal cloud, expressed as a fraction of the stellar flux. The proportionality factor is the transmission by the sky-projected KIN null fringe pattern, which is discussed in the next section. Thus, for a pointsource star with no circumstellar material, we would measure $E=0$, and this fraction increases as more exozodiacal emission is spatially resolved. KIN measured excess leak curves are accessible either per individual scan, per cluster (averaging several scans gathered within a single night), or by averaging data from different clusters/observing nights, when applicable. Excess leaks were computed for all 47 targets observed. For 44 of them (Figure 1), little or no significant fluctuation is detected between the different scans and all available data are averaged together as described above. The remaining three targets are small-separation multiple-star systems exhibiting large null variation versus time as the projected baseline or the companion position varies. In those cases, we show individual scan results (Figure 2) rather than temporal averages. Further details on multiple-system observations are given in Section 3.3. 

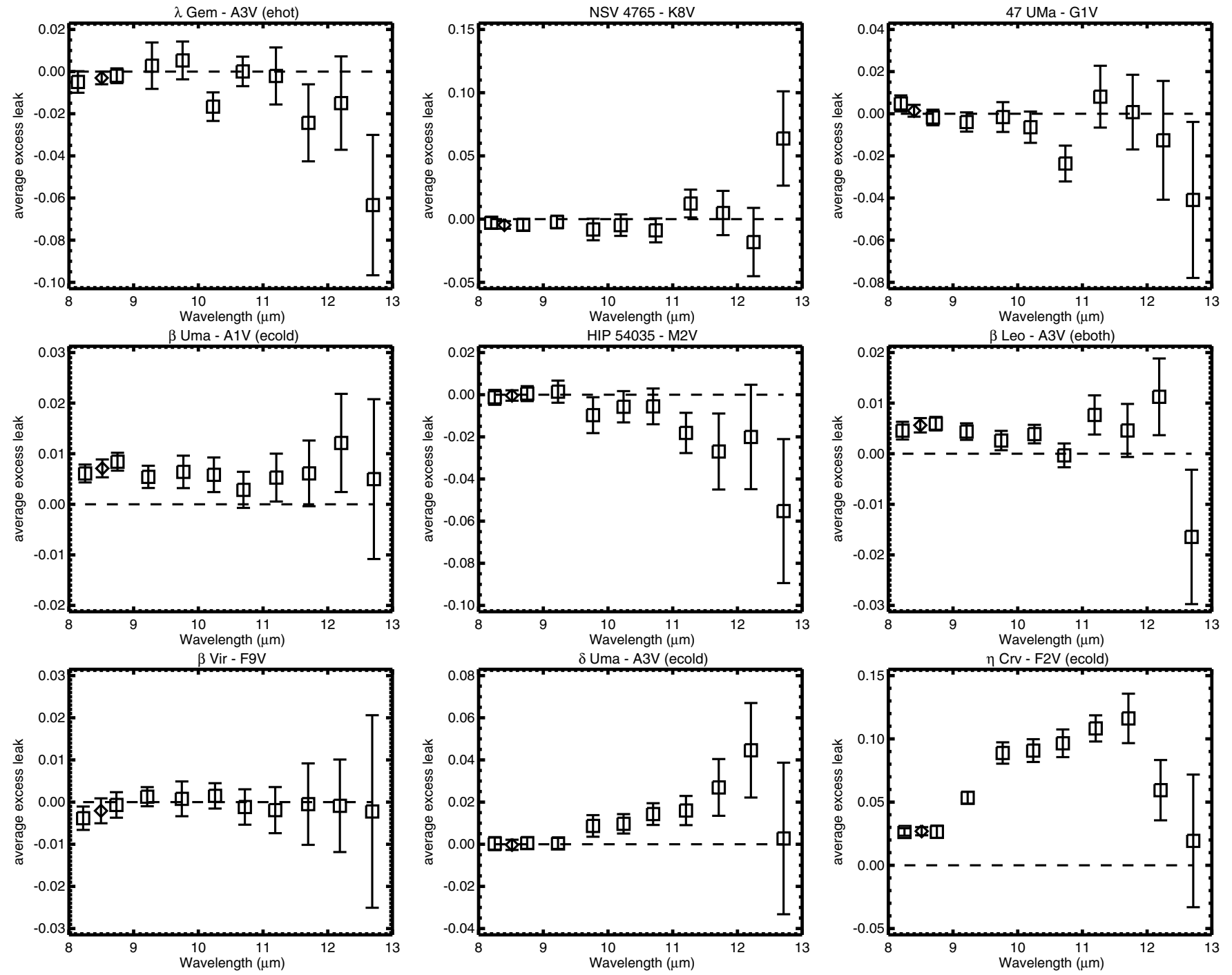

Figure 1. (Continued)

\subsection{Excess Leak Versus Physical Excess}

It is important to note that in order to convert a measured excess leak into an actual physical excess, the brightness distribution of the excess source must be known. In particular, because of the interference pattern and the limited field of view (FOV) of the instrument, the KIN-measured excess leaks are necessarily smaller than the actual astrophysical excesses. A complete description of these effects is given in separate publications (Serabyn et al. 2012; Mennesson et al. 2013), and a reminder of the relationship between measured leak and sky brightness distribution is given in Appendix A. In particular, any source of circumstellar excess lying (1) inside of the 6 mas KIN inner working angle (IWA), defined as the half transmission point at $10 \mu \mathrm{m}$, or (2) outside of its $\simeq 200$ mas outer working angle (OWA), set by the instrument 400 mas FWHM FOV, will be strongly attenuated or even completely missed. Using $300 \mathrm{~K}$ as a representative temperature of the habitable zone where future exo-Earth imaging missions will concentrate their efforts, and assuming dust emitting like a blackbody at thermal equilibrium with the star, we computed the $300 \mathrm{~K}$ dust location around all targets. For most stars in the sample (Table 1), the $300 \mathrm{~K}$ dust radial distance lies comfortably between the IWA and OWA: any extended dust emission at that temperature (or warmer) will be detected. For such stars, an attenuation factor of $\simeq 2$ is expected between the measured excess leak and the actual $10 \mu \mathrm{m}$ excess. ${ }^{12}$ For the nearest A and F stars, however, the $300 \mathrm{~K}$ dust radius lies outside of the KIN FOV, and a significant fraction of the excess may remain undetected by the KIN. This is especially true for Vega, Altair, $\alpha$ Psa, $\beta$ Leo, $\beta$ Cas, and $\beta$ UMa, all having hypothetical $300 \mathrm{~K}$ (or colder) dust located well outside of the KIN FOV.

\subsection{Binary Stars}

The KIN geometric FOV radius is limited to 300 mas by a pinhole located in an intermediary focal plane, yielding an FWHM of $\simeq 400$ mas when taking propagation, diffraction, and scattering effects into account (Colavita et al. 2013). Assuming an equal-brightness binary system, we find that any companion located $>5^{\prime \prime}$ away will have negligible impact on the KIN signal and can be safely ignored in the data processing. Using the Washington Visual Double Star Catalog (Mason et al. 2001) and the Sixth Catalog of Orbits of Visual and Binary Stars (Hartkopf et al. 2001), we find, however, that 9 of the 47 target stars had stellar companions within $5^{\prime \prime}$ at the time of the

\footnotetext{
12 This factor corresponds to the average transmission value over the region extending from the IWA to the OWA.
} 

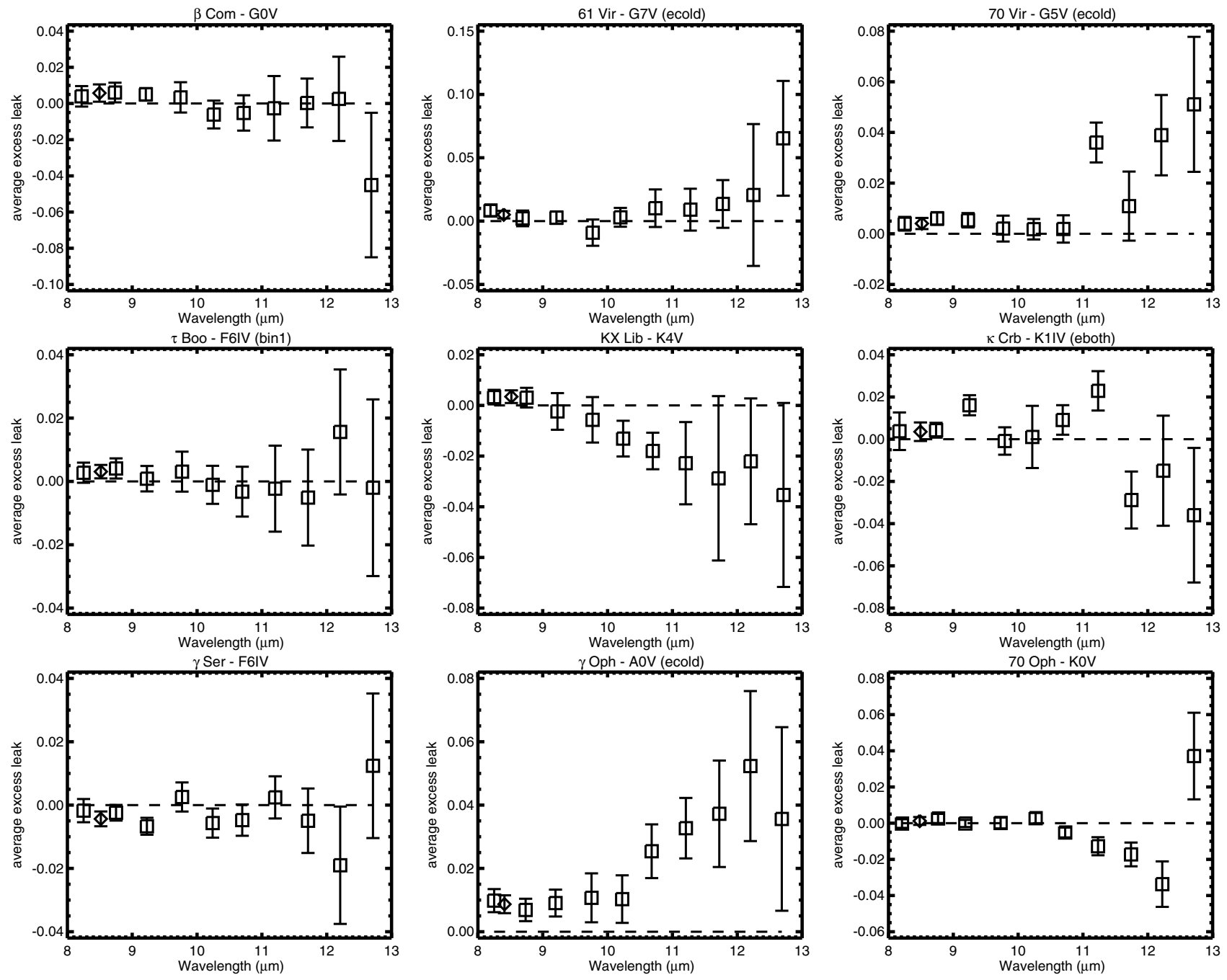

Figure 1. (Continued)

observations. The presence of these nearby companions affects data analysis at different levels depending on individual system's characteristics:

1. Both $\chi^{1}$ Ori and $\tau$ Boo have stellar companions within $5^{\prime \prime}$, but with no effect expected (or detected) on the measured leak. These two sources are indicated as type "bin 1" in Table 1. $\chi^{1}$ Ori is a single-line spectroscopic and astrometric binary with a faint companion $(\simeq 1.5 \%$ flux ratio at $H$ band; Köenig et al. 2002) located 700 mas away at the time of the KIN observations (Hartkopf et al. 2001). Adopting the effective temperatures derived for the two components $(5920 \mathrm{~K}$ and $3200 \mathrm{~K}$ ) and assuming blackbody stellar emission, the flux ratio is still only $3 \%$ at $10 \mu \mathrm{m}$. This means that the companion contributes negligible flux inside the FOV compared to the $\simeq 0.2 \% \mathrm{KIN}$ measurement uncertainty level. The astrometric companion to $\tau$ Boo has been directly imaged in 2001 at a separation of $2^{\prime \prime} .7$ with a relative flux of $1 \%$ at $800 \mathrm{~nm}$ (Roberts et al. 2011), corresponding to $\simeq 4 \%$ at $N$ band when adopting the F6IV/ M2V spectral types of Hale (1994) and assuming blackbody stellar emission. The separation predicted at the time of the KIN observations is 1".6 (Hartkopf et al. 2001), still far enough that no significant contribution to the null signal is expected from this faint companion. As far as the KIN measurements are concerned, these two stars are then effectively single, bringing up the total of KIN "single" stars to 40 .

2. For $\iota$ Peg, the companion is well inside the KIN FOV, but we found no obvious signature from a resolved companion in the leak excess curves, i.e., no variations with time or projected baseline. However, the companion is relatively bright, with a flux ratio of $4: 1$ at $N$ band, and may impact the measured null depth. We used the orbit and stellar system parameters of Boden et al. (1999) to determine the companion location at the two epochs of the observations and found separations of 2.38 and 2.54 mas, i.e., significantly smaller than the spatial resolution of the nuller. Computing the leak expected from this binary system, we found that the companion caused an extra leakage of $8 \times 10^{-4}$ at $8 \mu \mathrm{m}$ and even less at longer wavelengths. ८ Peg's excess leak curve shown in Figure 1 has been corrected for this small effect ("bin 2" object type in Table 1).

3. For $61 \mathrm{Cyg} \mathrm{A}, \delta$ Tri, and $\beta$ Tri, no obvious signature was found either in the measured leak excess curves, versus time or wavelength. We used published orbits from the Sixth Catalog of Orbits of Visual and Binary Stars (Hartkopf et al. 2001) to compute the companion locations at the epochs of the KIN observations. These companions are all well inside the KIN FOV (and located in the "positive leakage" area; see Appendix A and Figure 11). However, 

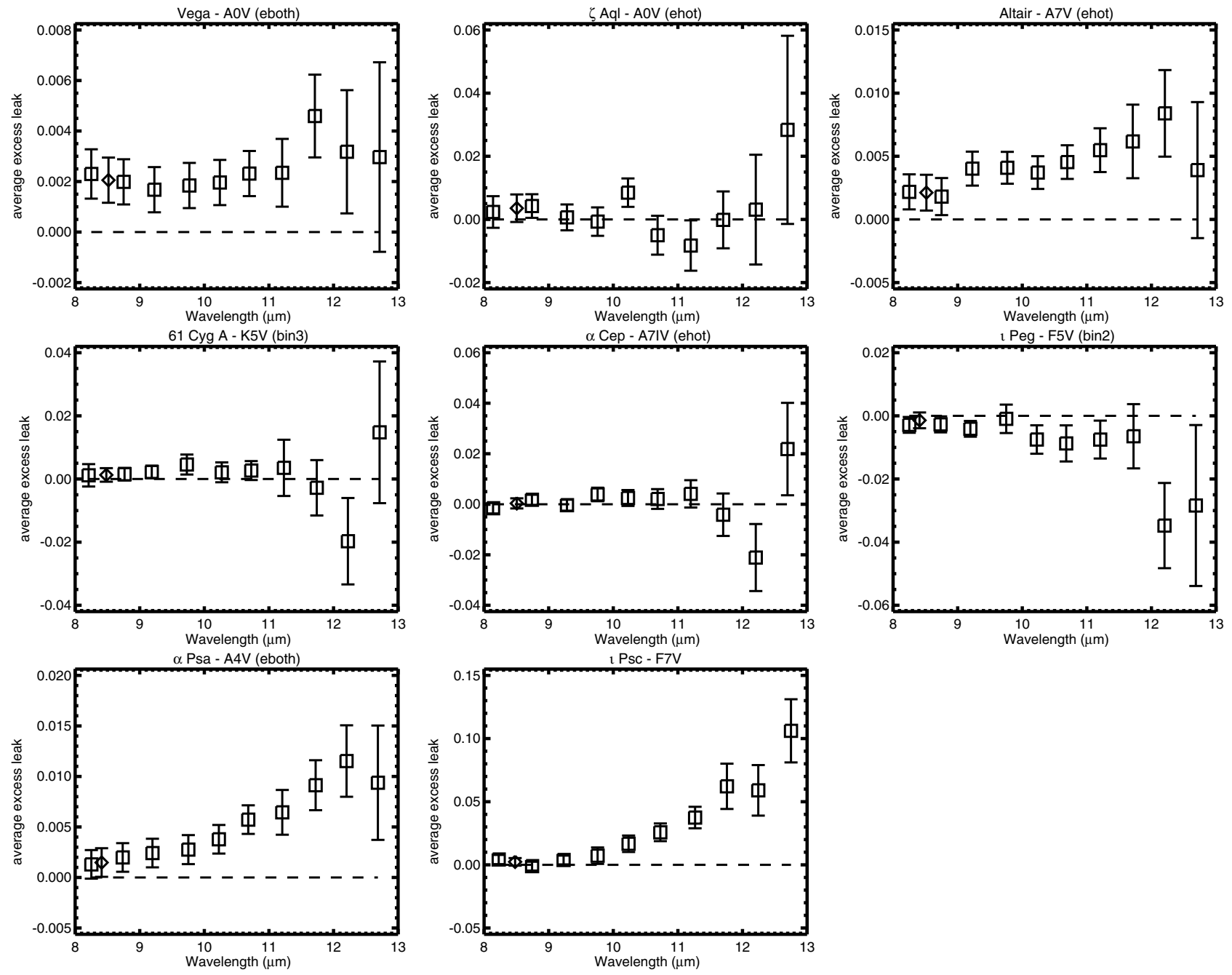

Figure 1. (Continued)

and unlike the case of $\iota$ Peg, we have found no information from which the component's flux ratio could be derived ("bin 3" object type in Table 1). Therefore, we perform no additional correction to account for the presence of a known companion in these systems. Subtracting the companion's contribution to the leak would only reduce the inferred leak excess and corresponding exozodiacal level, and thus any results derived for these stars, whether the excess leaks shown in Figure 1 or the zodi levels listed in Table 2, are to be interpreted as upper limits.

4. HD 83808, $\alpha \mathrm{Crb}$, and $\beta$ Per (the triple system Algol) have clear signatures of companions in the excess leaks measured (Figure 2), showing very large fluctuations versus hour angle, between $4 \%$ and $15 \%$ peak to peak. Given the uncertainties in the nuller off-axis transmission values and binary system parameters (companion position, diameter, and relative flux), the effect of these companions cannot be removed with high precision. As a result, these three stars are discarded from any further derivation of circumstellar dust excess and exozodi level ("bin 4" object type in Table 1).

The effective KIN exozodi sample is then reduced to 44 stars: 40 effectively single for KIN, one binary system with accurate dust excess determination ( $\iota$ Peg), and three more binary systems for which only upper limits are derived (61 Cyg A, $\delta$ Tri, and $\beta$ Tri).

\subsection{8-9 $\mu m$ Excesses}

As can be seen in all panels of Figure 1, the excess measurement uncertainty increases sharply with wavelength across the bandpass. The reason is that various instrumental factors (finite diffraction effects, material absorption, and pinhole mode matching) and the increased thermal background strongly reduce the KIN spectral sensitivity toward the red end of the bandpass. In addition, atmospheric dispersion is actively corrected around $9 \mu \mathrm{m}$, yielding larger chromatic effects and null uncertainties at longer wavelengths (Colavita et al. 2009). Under these conditions, the most reliable excess estimates come from measurements obtained in the $8-9 \mu \mathrm{m}$ bin. The quantity of interest is the ratio of the measured leak excess to its uncertainty, which we refer to hereafter as the "excess significance." It is worth noting that as a result of instrumental noise, the measured leak excess can be negative, and the excess significance as well. All excess measurements are summarized in Table 2 and show that five stars have an $8-9 \mu \mathrm{m}$ excess leak with a significance larger than $3 \sigma$. Among them, only $\eta \mathrm{Crv}$ shows a large $(8 \sigma)$ excess. The other four are $\beta \mathrm{UMa}, \beta \mathrm{Leo}, \zeta \mathrm{Lep}$, and $\gamma \mathrm{Oph}$, all showing excesses at the $3 \sigma-4 \sigma$ level. While $8-9 \mu \mathrm{m}$ excesses around $\eta \mathrm{Crv}$ and $\gamma$ Oph were already reported in Paper I, KIN excesses around the other three stars are reported here for the first time. Noticeably, all five stars had FIR excesses previously reported. A more detailed analysis of these five stars is given in Section 5.1, including their full 8-13 $\mu \mathrm{m}$ measurements. 

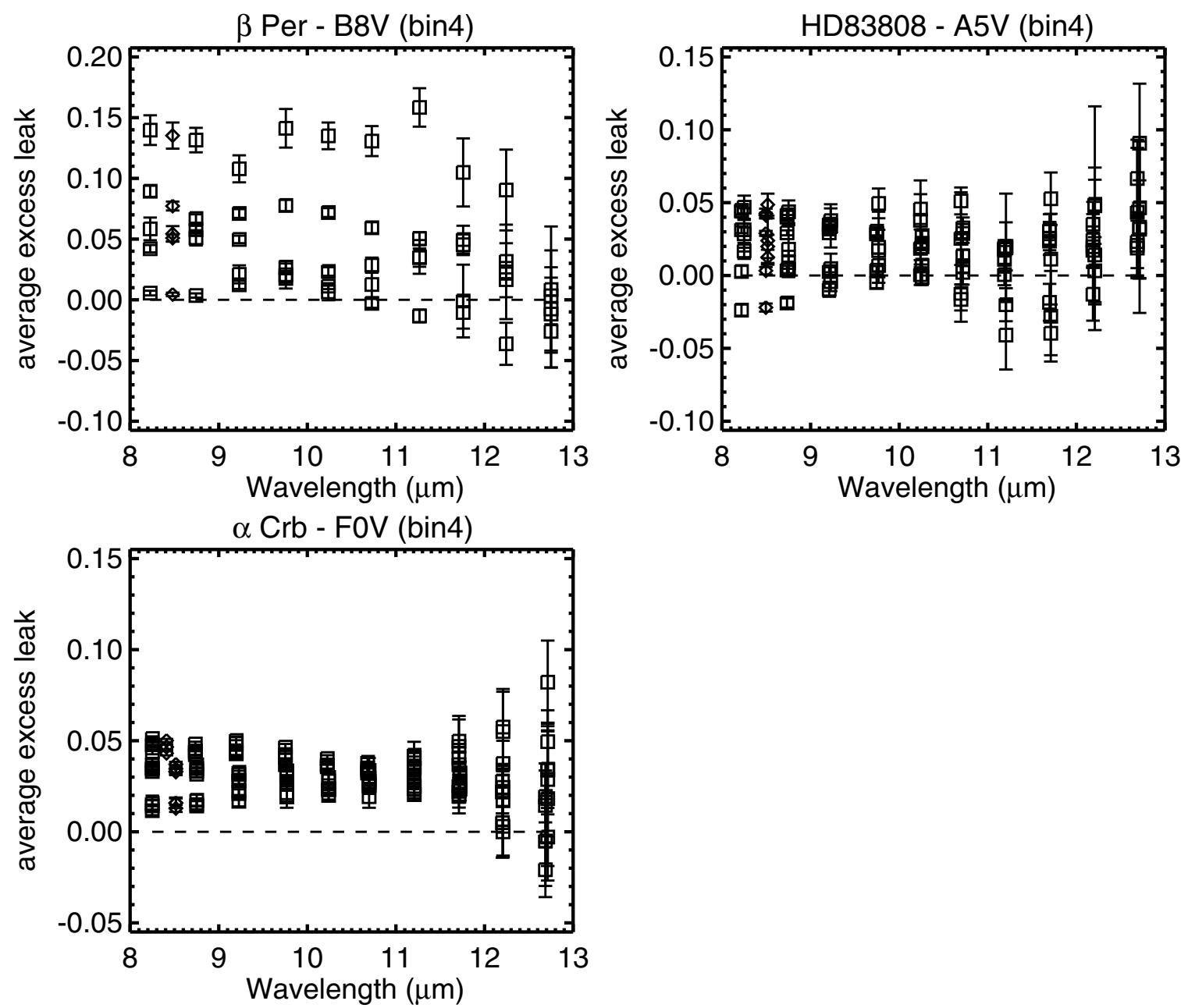

Figure 2. Excess leaks measured by the KIN for three stars with obvious signatures of stellar companions in the data (type "bin 4"). All available measurements are displayed, showing large null fluctuations vs. time and baseline azimuth. These stars have been discarded from subsequent zodiacal dust level estimations and correspond to the last three targets listed in Table 1.

\subsection{Zodi Levels}

In order to interpret the excess measurements and compare them with the solar system case, we used the Zodipic code ${ }^{13}$ to create images of zodi clouds around each target. Zodipic synthesizes brightness distributions of exozodiacal clouds based on the empirical fits to the observations of the solar zodiacal cloud made by COBE (Kelsall et al. 1998). When Zodipic generates a model brightness distribution for a zodi disk analog around a star other than the Sun, the dust has the same optical depth at $1 \mathrm{AU}$ and the same radial density profile as in the solar system. As a convenient unit, we refer to this model as corresponding to 1 "zodi." Zodipic scales the radial temperature profile with stellar luminosity, and the inner dust radius is set by a dust sublimation temperature of $1500 \mathrm{~K}$. The dust inner radius is thus dependent on stellar spectral type and ranges from $0.004 \mathrm{AU}$ for the coolest star in our sample (the M2V star HIP 54035) to $0.18 \mathrm{AU}$ for Vega. In the Zodipic code, the dust density can be treated as a free parameter, allowing generation of brightness distributions for a scaled version of the solar system (the total flux due to the circumstellar dust scales linearly with zodi level). Since the $8-9 \mu \mathrm{m}$ KIN measurements are of higher quality, we restricted the zodi-level calculations to that spectral bin, which

\footnotetext{
13 http://ssc.spitzer.caltech.edu/dataanalysistools/tools/contributed/
} general/zodipic/ also ensures continuity with the results derived in Paper I. In order to convert the measured 8-9 $\mu \mathrm{m}$ excess leaks to number of zodis, we used the procedure described in Section 4.2 of Paper I, summarized below. A one zodi dust cloud image (not including the central star) is generated around each star considered using the Zodipic model, and the overall dust flux transmitted through the instantaneous $(8.5 \mu \mathrm{m})$ KIN transmission pattern (see Appendix A) is computed. The resulting flux is then divided by the $8.5 \mu \mathrm{m}$ stellar flux, yielding the leak excess expected for a one zodi exozodical dust cloud, and a final estimate of the zodi level is required to match the observed excess leak. Since the dust cloud inclination and phase angle are generally unknown (except in a few cases; see Section 5.1), this operation is repeated for different exozodiacal disk orientations, from face-on to edge-on, with P.A. parallel and perpendicular ${ }^{14}$ to the instantaneous direction of the long-baseline fringes. These orientation effects are small compared to the KIN excess measurement error bars, but they are included in all derived zodi levels and uncertainties, which are listed in Table 2. We note that, although unphysical, negative zodis are allowed as a result of the error bars on the leak measurements. This procedure is exactly the same as used in Paper I (Section 4.2). We note, however, that in

\footnotetext{
14 Because of the finite scale height of the zodi dust cloud and the high spatial resolution of the KIN, an edge on-disk still contributes a significant null excess whatever the baseline orientation.
} 
Table 2

KIN Results Summary in the Context of Other IR Excess Measurements

\begin{tabular}{|c|c|c|c|c|c|c|c|c|c|c|c|}
\hline Star & $E_{8-9}$ & $\sigma_{8}-9$ & $S_{8-9}$ & Zodis & $E_{8-13}$ & $\sigma_{8-13}^{\text {uncor }}$ & $S^{\text {uncor }}$ & $\sigma_{8-13}^{\text {cor }}$ & $S^{\text {cor }}$ & $\begin{array}{c}\text { NIR/MIR/FIR } \\
\text { Excesses }\end{array}$ & $\begin{array}{c}T_{\text {cold }} \\
(\mathrm{K})\end{array}$ \\
\hline$\eta \mathrm{Crv}$ & $2.7 \mathrm{e}-02$ & $3.2 \mathrm{e}-03$ & 8.35 & $1813 \pm 209$ & $4.4 \mathrm{e}-02$ & $2.0 \mathrm{e}-03$ & 22.30 & $5.1 \mathrm{e}-03$ & 8.69 & $N^{\mathrm{a}} / Y^{\mathrm{b}} / Y^{\mathrm{c}}$ & 50 \\
\hline$\beta \mathrm{UMa}$ & $7.1 \mathrm{e}-03$ & $1.8 \mathrm{e}-03$ & 4.02 & $327 \pm 80$ & $6.4 \mathrm{e}-03$ & $9.2 \mathrm{e}-04$ & 7.00 & $2.5 e-03$ & 2.54 & $N^{\mathrm{a}} / Y^{\mathrm{b}} / Y^{\mathrm{c}, \mathrm{x}}$ & 140 \\
\hline$\beta$ Leo & $5.6 e-03$ & $1.4 \mathrm{e}-03$ & 3.96 & $291 \pm 73$ & $4.2 \mathrm{e}-03$ & $6.8 \mathrm{e}-04$ & 6.17 & $1.9 \mathrm{e}-03$ & 2.21 & $Y^{\mathrm{a}} / Y^{\mathrm{b}} / Y^{\mathrm{c}, \mathrm{d}}$ & 125 \\
\hline$\zeta$ Lep & $5.9 \mathrm{e}-03$ & $1.8 \mathrm{e}-03$ & 3.30 & $243 \pm 73$ & $9.6 e-03$ & $1.2 \mathrm{e}-03$ & 8.31 & $3.1 \mathrm{e}-03$ & 3.12 & $N^{\mathrm{a}} / Y^{\mathrm{b}} / Y^{\mathrm{e}}$ & 190 \\
\hline$\gamma \mathrm{Oph}$ & $8.7 \mathrm{e}-03$ & $2.8 \mathrm{e}-03$ & 3.08 & $306 \pm 99$ & $1.1 \mathrm{e}-02$ & $1.9 \mathrm{e}-03$ & 5.96 & $5.1 \mathrm{e}-03$ & 2.22 & $N^{\mathrm{a}} / Y^{\mathrm{f}} / Y^{\mathrm{f}}$ & 80 \\
\hline Vega & $2.1 \mathrm{e}-03$ & $8.9 \mathrm{e}-04$ & 2.30 & $297 \pm 124$ & $2.2 \mathrm{e}-03$ & $3.4 \mathrm{e}-04$ & 6.32 & $1.0 \mathrm{e}-03$ & 2.13 & $Y^{\mathrm{a}} / Y^{\mathrm{e}, \mathrm{g}} / Y^{\mathrm{e}}$ & 90 \\
\hline$\epsilon$ Eri & $2.5 \mathrm{e}-03$ & $1.2 \mathrm{e}-03$ & 2.16 & $262 \pm 117$ & $1.8 \mathrm{e}-03$ & $5.0 \mathrm{e}-04$ & 3.61 & $1.4 \mathrm{e}-03$ & 1.26 & $N^{\mathrm{a}} / Y^{\mathrm{h}} / Y^{\mathrm{h}}$ & 55 \\
\hline$\lambda$ Aur & $6.2 \mathrm{e}-03$ & $3.0 \mathrm{e}-03$ & 2.06 & $473 \pm 226$ & $5.6 e-03$ & $2.3 \mathrm{e}-03$ & 2.40 & $6.2 \mathrm{e}-03$ & 0.91 & $\mathrm{~N} / \mathrm{N} / \mathrm{N}$ & \\
\hline $10 \mathrm{Tau}$ & $7.6 \mathrm{e}-03$ & $4.1 \mathrm{e}-03$ & 1.84 & $614 \pm 333$ & $2.4 \mathrm{e}-03$ & $3.2 \mathrm{e}-03$ & 0.76 & $8.8 \mathrm{e}-03$ & 0.28 & $Y^{\mathrm{a}} / \mathrm{N} / Y^{\mathrm{i}}$ & 100 \\
\hline 70 Vir & $4.0 \mathrm{e}-03$ & $2.2 \mathrm{e}-03$ & 1.84 & $397 \pm 208$ & $5.6 \mathrm{e}-03$ & $1.3 \mathrm{e}-03$ & 4.30 & $3.5 \mathrm{e}-03$ & 1.60 & $N^{\mathrm{a}} / Y^{\mathrm{j}} / Y^{\mathrm{k}, l}$ & 65 \\
\hline $61 \mathrm{Vir}$ & $5.1 \mathrm{e}-03$ & $3.0 \mathrm{e}-03$ & 1.70 & $381 \pm 221$ & $4.6 \mathrm{e}-03$ & $2.5 \mathrm{e}-03$ & 1.81 & $6.6 \mathrm{e}-03$ & 0.69 & $\mathrm{~N} / N^{\mathrm{i}} / Y^{\mathrm{i}, \mathrm{m}}$ & 70 \\
\hline$\eta$ Cas A & $3.1 \mathrm{e}-03$ & $2.0 \mathrm{e}-03$ & 1.55 & $267 \pm 172$ & $3.3 \mathrm{e}-03$ & $9.9 \mathrm{e}-04$ & 3.30 & $2.7 \mathrm{e}-03$ & 1.21 & $N^{\mathrm{a}} / \mathrm{N} / \mathrm{N}$ & \\
\hline Altair & $2.1 \mathrm{e}-03$ & $1.4 \mathrm{e}-03$ & 1.50 & $247 \pm 176$ & $3.8 \mathrm{e}-03$ & $5.1 \mathrm{e}-04$ & 7.57 & $1.5 \mathrm{e}-03$ & 2.55 & $Y^{\mathrm{a}} / N^{\mathrm{n}} / N^{\mathrm{o}}$ & \\
\hline$\tau$ Boo & $3.1 \mathrm{e}-03$ & $2.1 \mathrm{e}-03$ & 1.46 & $208 \pm 142$ & $2.1 \mathrm{e}-03$ & $1.7 \mathrm{e}-03$ & 1.24 & $4.5 \mathrm{e}-03$ & 0.47 & $\mathrm{~N} / N^{\mathrm{p}} / N^{\mathrm{q}}$ & \\
\hline 1 Ori & $3.0 \mathrm{e}-03$ & $2.1 \mathrm{e}-03$ & 1.41 & $215 \pm 151$ & $1.7 \mathrm{e}-03$ & $1.2 \mathrm{e}-03$ & 1.38 & $3.4 \mathrm{e}-03$ & 0.50 & $N^{\mathrm{a}} / \mathrm{N} / \mathrm{N}$ & \\
\hline KX Lib & $3.5 \mathrm{e}-03$ & $2.5 \mathrm{e}-03$ & 1.38 & $326 \pm 235$ & $-1.1 \mathrm{e}-03$ & $1.9 \mathrm{e}-03$ & -0.58 & $4.9 \mathrm{e}-03$ & -0.23 & $\mathrm{~N} / \mathrm{N} / \mathrm{N}$ & \\
\hline$\beta \mathrm{Com}$ & $5.8 \mathrm{e}-03$ & $4.8 \mathrm{e}-03$ & 1.20 & $389 \pm 326$ & $3.0 \mathrm{e}-03$ & $2.2 \mathrm{e}-03$ & 1.34 & $6.0 \mathrm{e}-03$ & 0.50 & $\mathrm{~N} / \mathrm{N} / \mathrm{N}$ & \\
\hline$\alpha$ Psa & $1.5 \mathrm{e}-03$ & $1.4 \mathrm{e}-03$ & 1.05 & $118 \pm 107$ & $3.7 \mathrm{e}-03$ & $5.4 \mathrm{e}-04$ & 6.96 & $1.6 \mathrm{e}-03$ & 2.34 & $Y^{\mathrm{r}} / Y^{\mathrm{s}} / Y^{\mathrm{t}}$ & 70 \\
\hline$\beta$ Cas & $2.1 \mathrm{e}-03$ & $2.0 \mathrm{e}-03$ & 1.03 & $226 \pm 216$ & $1.7 \mathrm{e}-03$ & $7.7 \mathrm{e}-04$ & 2.24 & $2.2 \mathrm{e}-03$ & 0.77 & $N^{\mathrm{a}} / \mathrm{N} / Y^{\mathrm{u}}$ & 120 \\
\hline$\zeta \mathrm{Aql}$ & $3.6 \mathrm{e}-03$ & $4.4 \mathrm{e}-03$ & 0.82 & $137 \pm 167$ & $1.8 \mathrm{e}-03$ & $1.7 \mathrm{e}-03$ & 1.04 & $5.0 \mathrm{e}-03$ & 0.36 & $Y^{\mathrm{a}} / \mathrm{N} / \mathrm{N}$ & \\
\hline$\kappa \mathrm{Crb}$ & $3.5 \mathrm{e}-03$ & $4.4 \mathrm{e}-03$ & 0.80 & $625 \pm 791$ & $6.4 \mathrm{e}-03$ & $2.2 \mathrm{e}-03$ & 2.91 & $5.9 \mathrm{e}-03$ & 1.08 & $Y^{\mathrm{a}} / \mathrm{N} / Y^{\mathrm{v}}$ & 60 \\
\hline$\iota \mathrm{Psc}$ & $2.4 \mathrm{e}-03$ & $3.0 \mathrm{e}-03$ & 0.79 & $183 \pm 230$ & $8.2 \mathrm{e}-03$ & $1.7 \mathrm{e}-03$ & 4.67 & $4.8 \mathrm{e}-03$ & 1.71 & $\mathrm{~N} / \mathrm{N} / \mathrm{N}$ & \\
\hline 107 Psc & $2.0 \mathrm{e}-03$ & $3.0 \mathrm{e}-03$ & 0.67 & $160 \pm 236$ & $8.3 e-03$ & $2.5 \mathrm{e}-03$ & 3.39 & $6.8 \mathrm{e}-03$ & 1.23 & $N^{\mathrm{a}} / \mathrm{N} / \mathrm{N}$ & \\
\hline 61 Cyg A* & $1.3 \mathrm{e}-03$ & $2.2 \mathrm{e}-03$ & 0.60 & $127 \pm 212$ & $2.1 \mathrm{e}-03$ & $1.0 \mathrm{e}-03$ & 2.00 & $2.8 \mathrm{e}-03$ & 0.74 & $N^{\mathrm{a}} / \mathrm{N} / \mathrm{N}$ & \\
\hline 70 Oph A & $1.2 \mathrm{e}-03$ & $2.2 \mathrm{e}-03$ & 0.56 & $136 \pm 237$ & $-1.1 \mathrm{e}-03$ & $9.9 \mathrm{e}-04$ & -1.09 & $2.8 \mathrm{e}-03$ & -0.39 & $N^{\mathrm{a}} / \mathrm{N} / \mathrm{N}$ & \\
\hline $47 \mathrm{UMa}$ & $1.4 \mathrm{e}-03$ & $2.8 \mathrm{e}-03$ & 0.50 & $105 \pm 207$ & $-1.8 \mathrm{e}-03$ & $2.0 \mathrm{e}-03$ & -0.91 & $5.3 e-03$ & -0.34 & $\mathrm{~N} / \mathrm{N} / \mathrm{N}$ & \\
\hline$\alpha$ Cep & $3.4 \mathrm{e}-04$ & $2.0 \mathrm{e}-03$ & 0.17 & $24 \pm 144$ & $9.1 \mathrm{e}-04$ & $9.7 \mathrm{e}-04$ & 0.93 & $2.7 \mathrm{e}-03$ & 0.34 & $Y^{\mathrm{a}} / \mathrm{N} / \mathrm{N}$ & \\
\hline$\delta \mathrm{UMa}$ & $-2.7 \mathrm{e}-04$ & $2.4 \mathrm{e}-03$ & -0.11 & $-15 \pm 140$ & $3.8 \mathrm{e}-03$ & $1.4 \mathrm{e}-03$ & 2.81 & $3.7 \mathrm{e}-03$ & 1.05 & $N^{\mathrm{a}} / Y^{\mathrm{e}} / Y^{\mathrm{e}}$ & 215 \\
\hline HIP 54035 & $-3.9 \mathrm{e}-04$ & $2.5 \mathrm{e}-03$ & -0.16 & $-53 \pm 366$ & $-2.6 \mathrm{e}-03$ & $2.0 \mathrm{e}-03$ & -1.35 & $5.2 \mathrm{e}-03$ & -0.50 & $\mathrm{~N} / \mathrm{N} / \mathrm{N}$ & \\
\hline$\eta$ Lep & $-5.6 e-04$ & $1.7 \mathrm{e}-03$ & -0.32 & $-34 \pm 113$ & $-3.9 \mathrm{e}-03$ & $1.1 \mathrm{e}-03$ & -3.62 & $2.8 \mathrm{e}-03$ & -1.37 & $Y^{\mathrm{a}} / Y^{\mathrm{p}} / Y^{\mathrm{l}}$ & 170 \\
\hline$v$ And & $-1.1 \mathrm{e}-03$ & $3.1 \mathrm{e}-03$ & -0.34 & $-76 \pm 226$ & $-8.3 \mathrm{e}-04$ & $1.9 \mathrm{e}-03$ & -0.43 & $5.2 \mathrm{e}-03$ & -0.16 & $N^{\mathrm{a}} / \mathrm{N} / \mathrm{N}$ & \\
\hline$\chi_{1}$ Ori & $-9.2 \mathrm{e}-04$ & $2.7 \mathrm{e}-03$ & -0.34 & $-60 \pm 190$ & $-8.2 \mathrm{e}-04$ & $1.3 \mathrm{e}-03$ & -0.63 & $3.6 \mathrm{e}-03$ & -0.23 & $\mathrm{~N} / \mathrm{N} / \mathrm{N}$ & \\
\hline$\tau$ Ceti & $-1.1 \mathrm{e}-03$ & $2.1 \mathrm{e}-03$ & -0.53 & $-95 \pm 180$ & $-8.4 \mathrm{e}-04$ & $1.2 \mathrm{e}-03$ & -0.68 & $3.3 e-03$ & -0.25 & $Y^{\mathrm{a}} / \mathrm{N} / Y^{\mathrm{w}}$ & 60 \\
\hline$\theta$ Per & $-1.6 \mathrm{e}-03$ & $2.8 \mathrm{e}-03$ & -0.56 & $-112 \pm 204$ & $3.7 \mathrm{e}-04$ & $1.7 \mathrm{e}-03$ & 0.22 & $4.5 \mathrm{e}-03$ & 0.08 & $N^{\mathrm{a}} / \mathrm{N} / \mathrm{N}$ & \\
\hline$\iota \mathrm{Peg}^{*}$ & $-1.4 \mathrm{e}-03$ & $2.5 \mathrm{e}-03$ & -0.57 & $-100 \pm 173$ & $-4.2 \mathrm{e}-03$ & $1.2 \mathrm{e}-03$ & -3.38 & $3.4 \mathrm{e}-03$ & -1.24 & $\mathrm{~N} / \mathrm{N} / \mathrm{N}$ & \\
\hline$\beta$ Vir & $-2.1 \mathrm{e}-03$ & $3.0 \mathrm{e}-03$ & -0.70 & $-170 \pm 243$ & $-3.7 \mathrm{e}-04$ & $1.2 \mathrm{e}-03$ & -0.31 & $3.3 e-03$ & -0.11 & $N^{\mathrm{a}} / \mathrm{N} / \mathrm{N}$ & \\
\hline$\kappa_{1} \mathrm{Cet}$ & $-3.6 e-03$ & $3.6 \mathrm{e}-03$ & -0.98 & $-249 \pm 255$ & $-8.5 \mathrm{e}-03$ & $2.3 e-03$ & -3.67 & $6.1 \mathrm{e}-03$ & -1.39 & $\mathrm{~N} / \mathrm{N} / \mathrm{N}$ & \\
\hline$\lambda \mathrm{Gem}$ & $-3.0 \mathrm{e}-03$ & $3.0 \mathrm{e}-03$ & -1.00 & $-139 \pm 140$ & $-4.1 \mathrm{e}-03$ & $2.3 \mathrm{e}-03$ & -1.80 & $6.1 \mathrm{e}-03$ & -0.67 & $Y^{\mathrm{a}} / \mathrm{N} / \mathrm{N}$ & \\
\hline$\delta T r i^{*}$ & $-3.7 \mathrm{e}-03$ & $3.0 \mathrm{e}-03$ & -1.24 & $-298 \pm 242$ & $-6.6 e-03$ & $1.9 \mathrm{e}-03$ & -3.44 & $4.5 e-03$ & -1.46 & $\mathrm{~N} / \mathrm{N} / \mathrm{N}$ & \\
\hline$\beta \operatorname{Tri}^{*}$ & $-6.5 \mathrm{e}-03$ & $4.7 \mathrm{e}-03$ & -1.39 & $-436 \pm 316$ & $-7.1 \mathrm{e}-04$ & $2.4 \mathrm{e}-03$ & -0.30 & $6.2 \mathrm{e}-03$ & -0.11 & $\mathrm{~N} / \mathrm{N} / Y^{\mathrm{o}}$ & 90 \\
\hline NSV 4765 & $-4.6 \mathrm{e}-03$ & $3.0 \mathrm{e}-03$ & -1.53 & $-477 \pm 316$ & $-3.1 \mathrm{e}-03$ & $2.3 \mathrm{e}-03$ & -1.37 & $6.3 e-03$ & -0.50 & $\mathrm{~N} / \mathrm{N} / \mathrm{N}$ & \\
\hline$\gamma$ Lep & $-3.0 \mathrm{e}-03$ & $1.8 \mathrm{e}-03$ & -1.67 & $-205 \pm 127$ & $-1.1 \mathrm{e}-03$ & $9.0 \mathrm{e}-04$ & -1.23 & $2.4 \mathrm{e}-03$ & -0.46 & $\mathrm{~N} / \mathrm{N} / \mathrm{N}$ & \\
\hline$\iota$ Per & $-4.5 \mathrm{e}-03$ & $2.5 \mathrm{e}-03$ & -1.82 & $-338 \pm 188$ & $-2.5 \mathrm{e}-04$ & $1.4 \mathrm{e}-03$ & -0.18 & $3.7 \mathrm{e}-03$ & -0.07 & $\mathrm{~N} / \mathrm{N} / \mathrm{N}$ & \\
\hline$\gamma$ Ser & $-4.4 \mathrm{e}-03$ & $2.3 e-03$ & -1.87 & $-304 \pm 164$ & $-3.3 e-03$ & $1.3 \mathrm{e}-03$ & -2.47 & $3.7 \mathrm{e}-03$ & -0.90 & $N^{\mathrm{a}} / \mathrm{N} / \mathrm{N}$ & \\
\hline
\end{tabular}

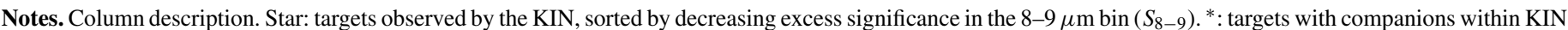

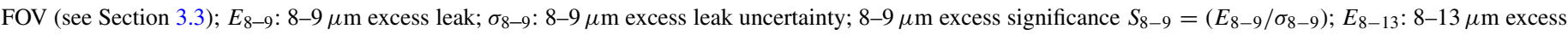

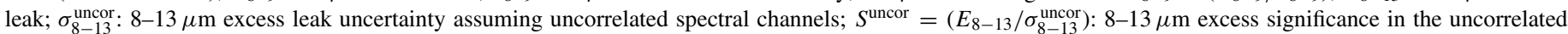

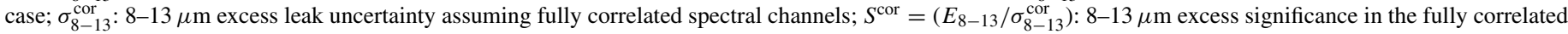

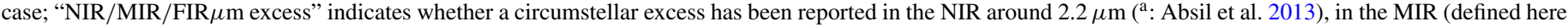

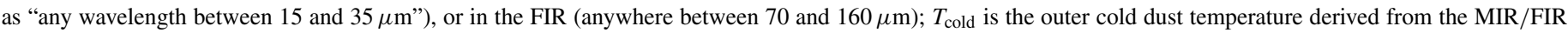

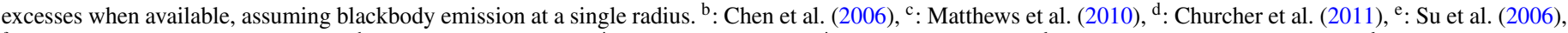

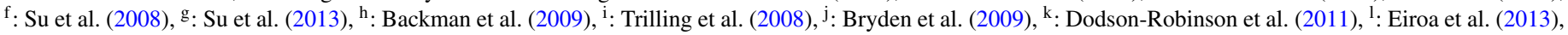

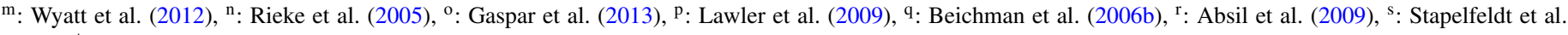
(2004), ${ }^{\text {t}}$ : Acke et al. (2012), ${ }^{\text {u}: ~ R h e e ~ e t ~ a l . ~(2007), ~}{ }^{\mathrm{v}}$ : Bonsor et al. (2013), ${ }^{\mathrm{w}}$ : Habing et al. (2001), ${ }^{\mathrm{x}}$ : Booth et al. (2013).

this paper we have used more accurate estimates of the stellar flux in the KIN 8-9 $\mu \mathrm{m}$ spectral bin. We have used Akari fluxes in the $9 \mu \mathrm{m}$ band, color corrected, and scaled to the $8.5 \mu \mathrm{m} \mathrm{KIN}$ effective wavelength using a Rayleigh-Jeans assumption. These revised stellar fluxes result in new estimates of the zodi levels for the 25 stars of Paper I, in average $40 \%$ higher, and supersede those of Paper I.

\subsection{8-13 $\mu \mathrm{m}$ Excesses}

In addition to the $8-9 \mu \mathrm{m}$ bin, we made use of the measurements obtained over the full $N$ band, with the goal of identifying possible new excess stars, i.e., stars with excess only present at the longest KIN wavelengths. To this end, we interpreted each of the 10 spectral channel excess leaks as individual 
measurements of a constant broadband 8-13 $\mu \mathrm{m}$ excess and formed an average. A difficulty in averaging such measurements is that some correlation is suspected between the different KIN spectral channels (Colavita et al. 2009), but that the actual spectral data covariance matrix is unknown. In this case where the exact correlation pattern is unknown, a robust way of estimating the mean is to ignore all correlation terms and use a simple weighted average (Schmelling 1995). Assuming that each spectral channel (index $k$ ) is characterized by a measured excess leak $E_{k}$ with $1 \sigma$ Gaussian error $\sigma_{k}$, we then estimated the broadband excess as

$$
E_{8-13}=\frac{\sum_{k} E_{k} / \sigma_{k}^{2}}{\sum_{k} 1 / \sigma_{k}^{2}} .
$$

The uncertainty on this estimated broadband excess is more difficult to assess. In order to bound the problem, and since correlation factors are unknown, we examine two extreme cases.

\subsubsection{Uncorrelated Spectral Channels}

If all spectral channel measurements were completely uncorrelated (the most optimistic scenario), the uncertainty on the broadband excess would be

$$
\sigma_{8-13}=\sqrt{\frac{1}{\sum_{k} 1 / \sigma_{k}^{2}}} .
$$

In this uncorrelated case, the broadband excess significance would then be given by

$$
S_{\text {uncor }}=\frac{E_{8-13}}{\sigma_{8-13}}=\frac{\sum_{k} E_{k} / \sigma_{k}^{2}}{\sqrt{\sum_{k} 1 / \sigma_{k}^{2}}} .
$$

In particular, if all 10 spectral channels had the same measurement uncertainty $\sigma_{k}=\sigma_{0}$ (a simple illustrative case to consider), one would get

$$
\begin{aligned}
E_{8-13} & =\left\langle E_{k}\right\rangle, \\
\sigma_{8-13} & =\frac{\sigma_{0}}{\sqrt{10}}, \\
S_{\text {uncor }} & =\sqrt{10} \frac{\left\langle E_{k}\right\rangle}{\sigma_{0}} .
\end{aligned}
$$

The broadband excess derived is simply the mean of observed values, and its significance increases as the square root of the number of spectral channels, as expected for uncorrelated data.

\subsubsection{Fully Correlated Spectral Channels}

Unfortunately, excess measurements obtained in different spectral channels are known to be partially correlated (Colavita et al. 2009), an effect believed to be caused by residual thermal background correlation at the single telescope cross-combiner level and by small differential dispersion effects between science targets and calibrators. In order to derive the most conservative error bar on the broadband excess, we assume here that the various spectral channel measurements are fully correlated (worst-case scenario). In that case, the uncertainty on the broadband excess is given by

$$
\sigma_{8-13}=\frac{\sum_{k} 1 / \sigma_{k}}{\sum_{k} 1 / \sigma_{k}^{2}},
$$

and the broadband excess significance becomes

$$
S_{\text {cor }}=\frac{E_{8-13}}{\sigma_{8-13}}=\frac{\sum_{k} E_{k} / \sigma_{k}^{2}}{\sum_{k} 1 / \sigma_{k}} .
$$

And if all 10 spectral channels had the same measurement uncertainty $\sigma_{k}=\sigma_{0}$, one would then get

$$
\begin{aligned}
E_{8-13} & =\left\langle E_{k}\right\rangle, \\
\sigma_{8-13} & =\sigma_{0}, \\
S_{\text {cor }} & =\frac{\left\langle E_{k}\right\rangle}{\sigma_{0}} .
\end{aligned}
$$

Compared to the uncorrelated case above, the uncertainty is now higher by $\sqrt{10}$, and the broadband excess significance is reduced by this factor. Table 2 summarizes the broadband $8-13 \mu \mathrm{m}$ excess leak measured for each star, with the uncertainties and significances derived in both the uncorrelated and fully correlated cases. The former is only given for reference (bestcase scenario). Only the values derived in the fully correlated case (worst-case scenario) are considered for determining a potential 8-13 $\mu \mathrm{m}$ excess and for further statistical analysis.

\subsubsection{8-13 $\mu \mathrm{m}$ Excess Stars}

Based on the results of Table 2, and assuming that all spectral channel measurements are fully correlated, only two stars show a broadband excess with a significance larger than $3 \sigma: \eta \mathrm{CrV}$ and $\zeta$ Lep. However, the statistical analysis conducted in the next section suggests that many more stars have a broadband excess, albeit close to the detection limit of the KIN.

\section{STATISTICAL ANALYSES}

Rather than looking at the results for individual stars, we use here the whole sample or specific subgroups of it. The goal is to obtain more sensitive estimates of the number of stars with a MIR excess and look for possible statistical trends, such as correlation of the observed KIN excesses with basic stellar properties. In order to avoid any possible contamination of the null measurements, all further analyses are limited to the 40 "effectively single" stars, as defined in Section 3.3.

\subsection{8-9 $\mu m$ Excess Distribution}

Figure 3(a) shows the histogram of excess significance measured in the 8-9 $\mu \mathrm{m}$ bin. As already discussed in Section 3.3, five stars show an excess significance greater than 3 and have hence a formally detected KIN excess. But as can be readily seen, the observed distribution tells more: it is highly nonsymmetrical and shows a tail toward larger excesses. Excess measurements with a "negative significance" are a consequence of the measurement noise, but this also means that such data can be used to infer the instrumental noise distribution. The dotted line shows the best Gaussian fit to these negative significance histogram data (after symmetrizing around $x=0$ and taking into account the averaging due to finite bin size). The best-fit Gaussian noise distribution has a standard deviation of 1.002, very close to unity, as would be expected from the empirical standard deviation of many independent realizations of the variable $\left(E_{8-9} / \sigma_{E_{8-9}}\right)$. Further evidence for the validity of this instrumental noise estimation and its Gaussianity is given in Section 4.5. ${ }^{15}$ Although they cannot be formally identified as

\footnotetext{
15 As shown in Figure 4(a), which concentrates exclusively on the 20 stars with no infrared excess previously known, a zero-mean Gaussian distribution with a standard deviation close to unity is also found to be a very good fit to the data (also see Paper I).
} 

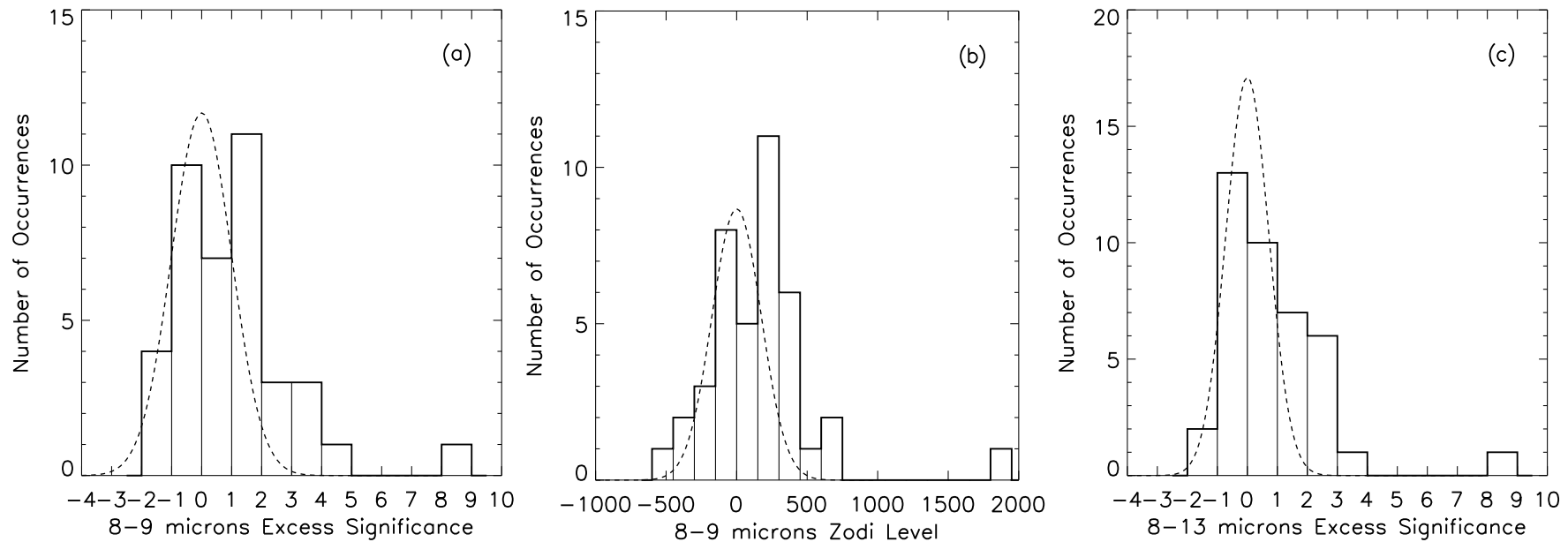

Figure 3. Statistical analysis. Histograms derived from the KIN measurements of 40 "effectively single" stars. (a) Histogram of the 8-9 $\mu$ m excess significance. The observed histogram is highly asymmetric and obviously skewed toward positive excesses, with five stars showing excesses detected at the $>3 \sigma$ level. (b) Histogram of exozodi levels (expressed in solar zodi units, 150 zodis per bin) derived from the KIN $8-9 \mu$ m measurements. The observed histogram is again asymmetric and obviously skewed toward zodi levels higher than the average measurement error of 200 zodis rms, showing a large number of measurements between 150 and 450 zodis. (c) Histogram of broadband 8-13 $\mu \mathrm{m}$ excess significance. Broadband excess error bars and significance are estimated assuming full correlation between the 10 spectral channels, which is the worst-case scenario. The observed histogram is also asymmetric with a tail extending toward positive excesses, two stars showing excesses detected at the $>3 \sigma$ level, and six more above $2 \sigma$. For each plot, the dashed line indicates for comparison the instrumental noise distribution derived from the data, assuming Gaussian behavior (see Sections 4.1-4.3 for details).

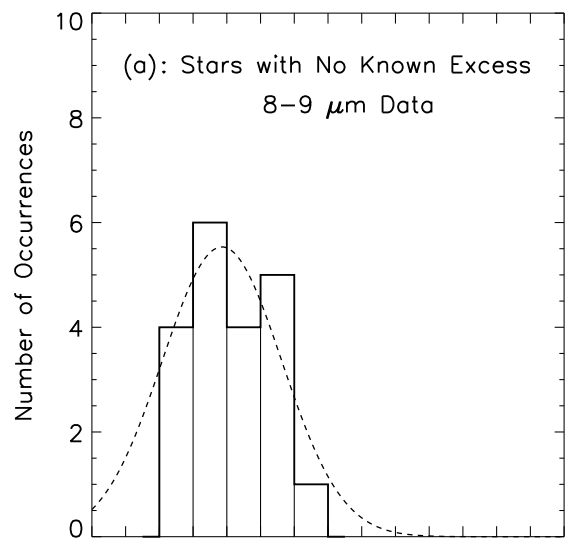

$-4-3-2-1001253 \quad 4566788910$ 8-9 microns Excess Significance

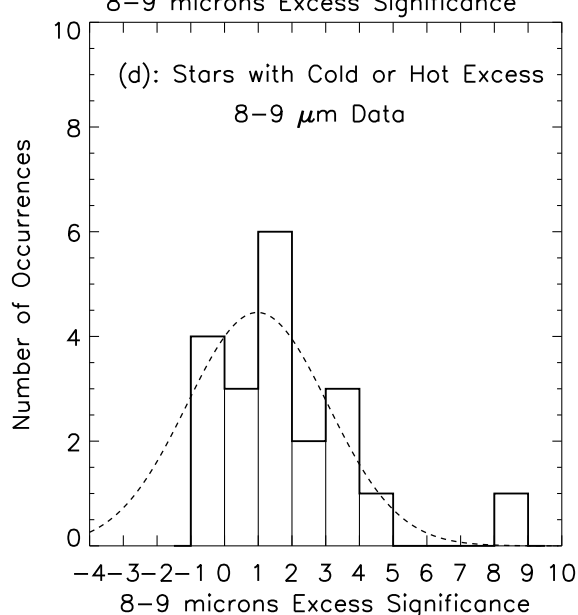

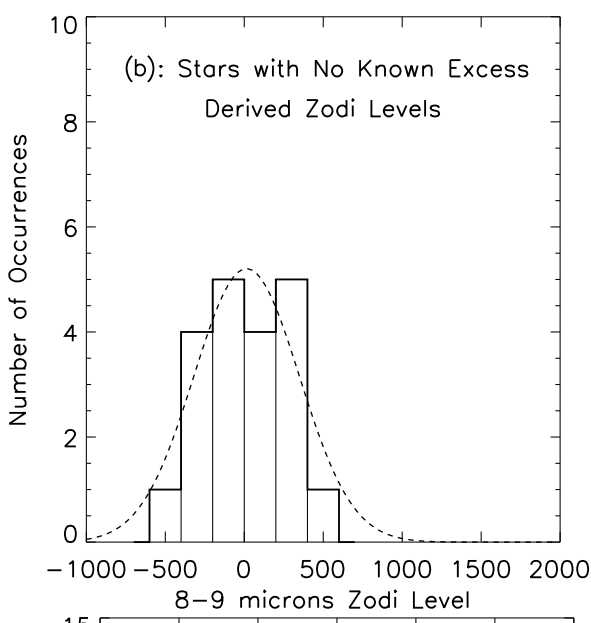

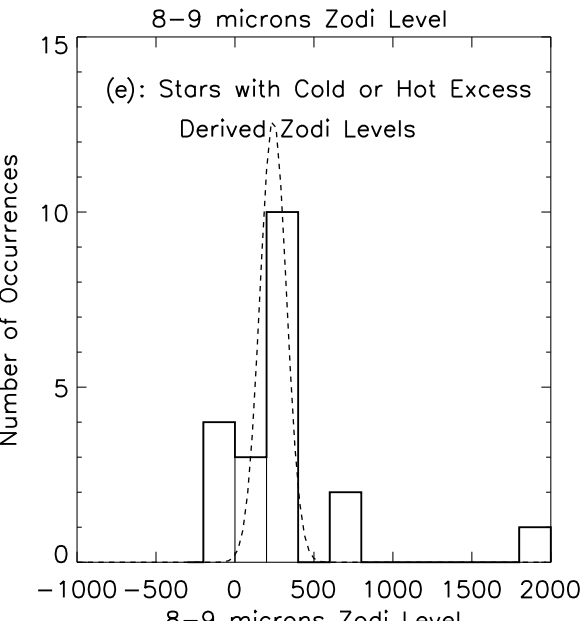

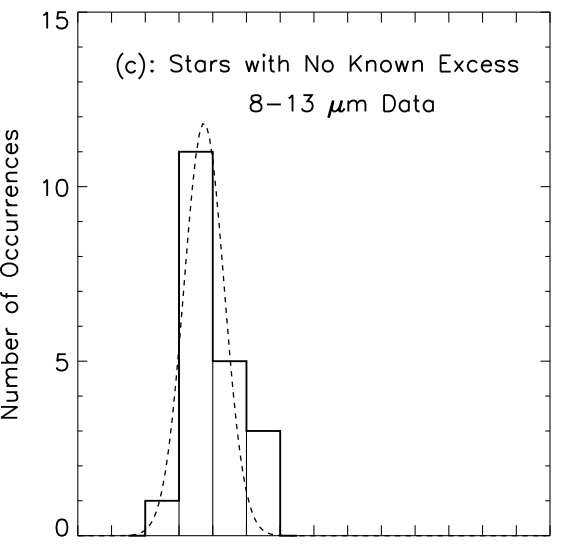
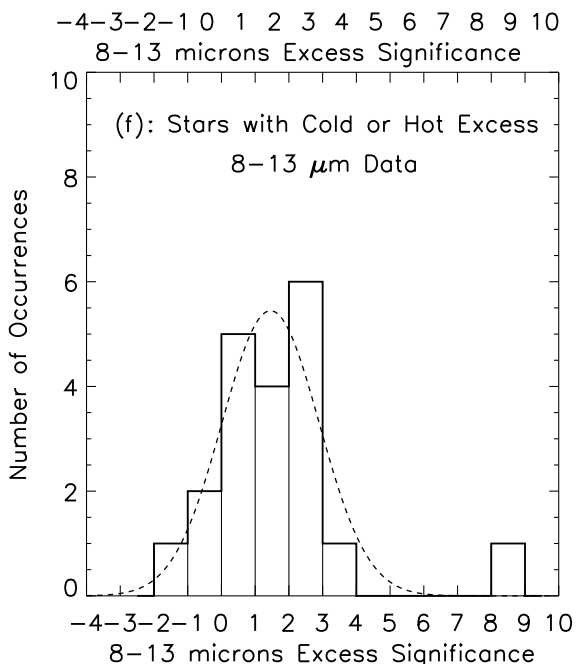

Figure 4. Comparing KIN results for stars with no infrared excesses previously known (top panels) and stars with cold (FIR) or hot (NIR) infrared excesses previously detected (bottom panels). Each subgroup is composed of 20 stars. ((a) and (d)) Histograms of the measured 8-9 $\mu$ m excess significance. ((b) and (e)) Histograms of exozodi levels derived from the 8-9 $\mu \mathrm{m}$ measurements, where each bin is 200 solar zodis wide. ((c) and (f)) Histograms of the measured 8-13 $\mu \mathrm{m}$ excess significance. $\eta$ Crv appears as a clear outlier in all lower panel histograms, with a high excess significance ( $>8)$ and the largest measured zodi level (1870 zodis). For all panels, the dashed lines indicate the best-fit Gaussian distribution to the data (ignoring $\eta$ Crv for the lower panel fits). Stars with previously detected cold (FIR) or hot (NIR) excesses have observed distributions systematically shifted toward higher excess significance and zodi levels, with a high number of measurements concentrating between 200 and 400 solar zodis and three above 600 zodis (panel (e)). They are also the only stars to show $8-9 \mu$ m excesses detected above $3 \sigma$ (five stars in panel (d)) or $8-13 \mu \mathrm{m}$ excesses detected above $2 \sigma$ (eight stars in panel (f)). 
bona fide excess stars, there are several more targets with excess significance at the $1 \sigma-3 \sigma$ level than predicted by instrumental noise only: in addition to the five stars with significant excess, about 10 more stars lie close to the KIN detection limit and would be interesting targets for an instrument providing better contrast, even by just a factor of $\simeq 3$.

\subsection{Exozodi Level Distribution}

Figure 3(b) shows the histogram of zodi levels derived around the 40 stars in the sample. Here again, the dotted line shows the best-fit Gaussian to the negative measurements, providing an estimate of the measurement noise distribution. Its standard deviation is 190 zodis, a value very close to the mean and median zodi measurement uncertainty of the overall sample (202 and 188 zodis, respectively). The zodi level and the excess significance are not necessarily related, since zodi level and uncertainty levels are a priori uncorrelated (e.g., a large zodi level might be associated with a large uncertainty and hence a low detection confidence level.) This means that the excess significance and zodi-level histograms provide independent information, at least to some extent. However, and quite interestingly, a similar behavior is found in the empirical histograms of both quantities. Once again, there seem to be significantly more than five stars with zodi levels higher than what the noise distribution predicts. This excess is particularly visible between 150 and 450 zodis.

\subsection{Broadband Excess Distribution}

Figure 3(c) shows the histogram of excess significance measured between 8 and $13 \mu \mathrm{m}$, assuming that all spectral channels are fully correlated. This assumption yields the lowest possible excess significance levels given the data, and hence the most conservative estimates of broadband excess detection. While only two stars ( $\eta$ Crv and $\zeta$ Lep) show formally detected correlated broadband excesses with a significance greater than $3 \sigma$, the observed distribution is again very asymmetric, and the number of stars with significance between $1 \sigma$ and $3 \sigma$ again exceeds the levels predicted by pure instrumental noise. In addition, the best-fit noise distribution (dotted curve) derived from "negative excess" data has a standard deviation of 0.68 . This low value suggests that the broadband excess significance estimates are generally underestimated by a factor of $\simeq 1.5$. In other words, any star with a correlated broadband excess quoted at the $2 \sigma$ level is more likely a $3 \sigma$ excess. Some further evidence for overestimated error bars comes from the analysis of the subsample of 20 stars with no infrared excess previously known, presented in Section 4.5. ${ }^{16}$ Applying a $2 \sigma$ cut instead of $3 \sigma$ (per the argument presented above) leaves eight sources: the five stars with 8-9 $\mu \mathrm{m}$ excess already listed in Section $3.3(\eta$ $\mathrm{Crv}, \beta \mathrm{UMa}, \beta$ Leo, $\zeta$ Lep, and $\gamma \mathrm{Oph}$ ), along with Fomalhaut ( $\alpha$ Psa), Altair, and Vega.

\subsection{Influence of Spectral Type and Age}

Considering the 8-9 $\mu \mathrm{m}$ excess significance measured as a function of spectral type, KIN excesses at the $3 \sigma$ level or higher are exclusively found around early-type stars. With the exception of $\eta \mathrm{Crv}$ (F2V type), all stars showing an 8-9 $\mu \mathrm{m}$ excess are A stars. Of course, the analysis suffers from small number statistics, but the observed fractions of excess stars

\footnotetext{
16 In this case, a zero mean Gaussian distribution with a standard deviation of 0.64 - again notably smaller than unity - is found to be the best fit to the broadband excess significance histogram (Figure 4(c)).
}

are very different at $4 / 11,1 / 13,0 / 9$, and $0 / 7$ for $\mathrm{A}, \mathrm{F}, \mathrm{G}$, and $\mathrm{K} / \mathrm{M}^{17}$ stars, respectively. Extending the analysis to the KIN broadband $8-13 \mu \mathrm{m}$ measurements, A stars are even more favored: among the eight stars showing an excess significance at the $2 \sigma$ level or higher, seven are A stars. This is in contrast with the results found for stars with no previously known infrared excess (Paper I, Appendix A), for which no correlation was found between excess leakage and stellar effective temperature. In fact, if any instrumental bias was affecting the measurements, it would rather tend to underestimate excesses around nearby $\mathrm{A}$ and early F stars. Indeed, while the KIN baseline is long enough to comfortably resolve the inner zodi regions of even the coldest and most distant stars in the sample, only nearby A and F stars have $300 \mathrm{~K}$ dust radii lying outside of the KIN OWA (Table 1). As discussed before (Section 3.2), this effect is precisely the strongest around Vega, Altair, $\alpha$ Psa, $\beta$ Leo, and $\beta$ UMa. This suggests that the small excess leak detected around some of these stars could actually trace a significantly larger MIR physical excess (see Section 5.2 for discussion of individual targets). The higher occurrence of $10 \mu \mathrm{m}$ excess observed around A stars appears then to be a real astrophysical trend. Since A stars have the shortest main-sequence lifetime, this apparent correlation of the KIN excess with spectral type could also be caused by a correlation with age. A similar trend was observed at FIR and near-infrared (NIR) wavelengths (Trilling et al. 2008; Absil et al. 2013).

\subsection{Connection with Excesses Detected at Other IR Wavelengths}

As summarized in Table 2 (last column), 20 of the KIN "single" stars have either a "cold" FIR $(>60 \mu \mathrm{m})$ excess or a "hot" NIR $(\simeq 2.2 \mu \mathrm{m})$ excess detected. While the former excess type is likely due to outer dust and comet reservoirs analogous to the solar system Kuiper Belt, the origin of the NIR excess is still poorly understood. The most likely explanation is the presence of submicron-sized hot dust particles piling up close to the sublimation radius of these stars (Absil et al. 2013; Mennesson et al. 2011; Lebreton et al. 2013). In order to help understand the origin of those various types of infrared excess, we correlated them with the detected KIN excess.

Figure 4 compares the distribution measured for stars with no previously known infrared excess (Group 1) with that obtained for stars with either a cold or a hot infrared excess previously reported (Group 2). Each group contains 20 stars each, allowing a straightforward comparison of the observed distributions. While distributions measured for the first group are centered around zero and fairly symmetric, the second-group distributions are heavily skewed toward positive detections. This effect is especially pronounced for the broadband KIN measurements (Figure 4, panels (c) and (f)). Indeed, while none of the 20 stars in Group 1 show a large broadband excess significance (all below a value of 1.7), eight of the Group 2 stars show a broadband excess significance larger than 2. A similar effect is seen between the zodi-level distributions of the two groups (Figure 4, panels (b) and (e)): stars in Group 2 have their observed distribution shifted toward higher zodi levels, exhibiting significantly more stars in the 200-400 zodi-level bin and at zodi levels above 600 zodis. Even after excluding $\eta$ Crv and its unusually bright MIR dust emission, the weighted mean of the zodi levels measured for Group 2 stars is 206

\footnotetext{
17 There was only one M star in the sample (HIP 54035, M2V spectral type). It was grouped with the six K stars for the purpose of this analysis.
} 


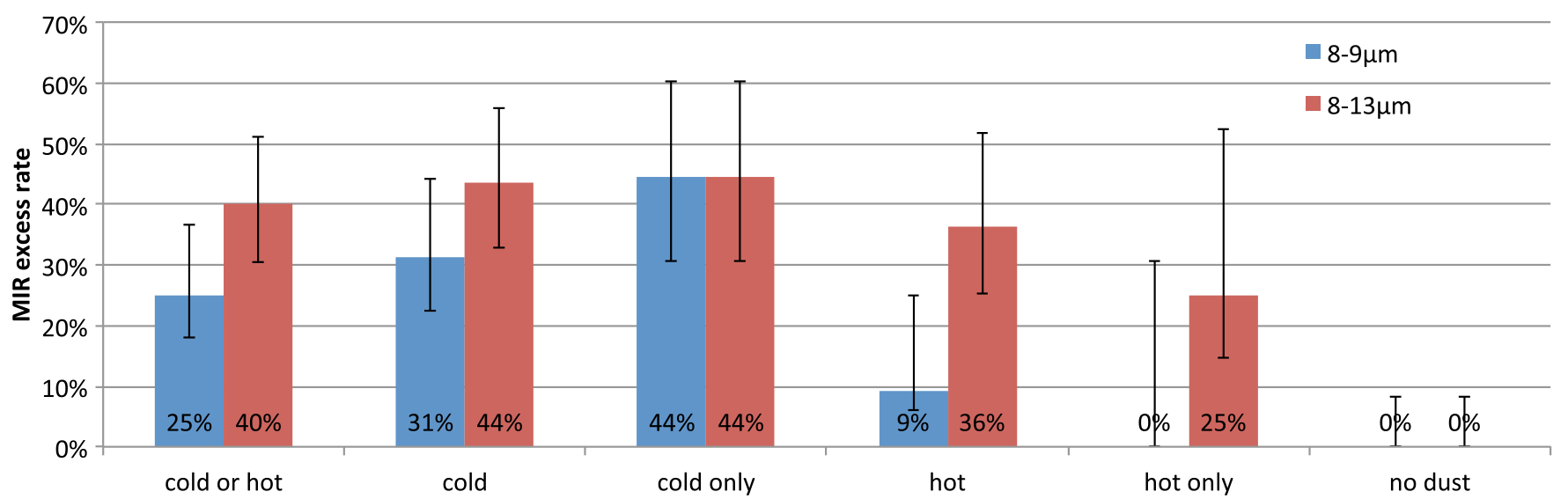

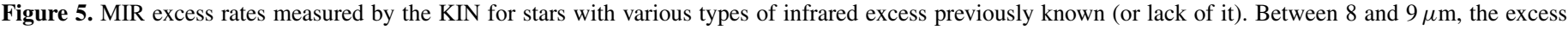

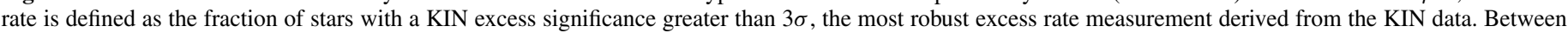

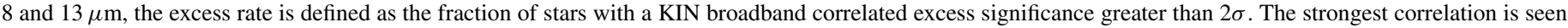

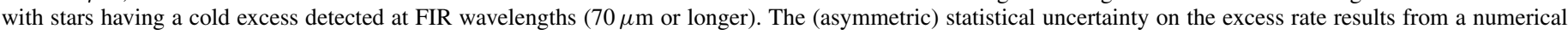
integration of the binomial distribution; see Section 4.5 for details.

(A color version of this figure is available in the online journal.)

Table 3

Summary of 8-9 $\mu \mathrm{m}$ Excess Rates Measured as a Function of Spectral Type and Previously Detected Infrared Excesses

\begin{tabular}{lccccc}
\hline \hline & $\mathrm{A}$ & $\mathrm{F}$ & $\mathrm{G}$ & $\mathrm{K} / \mathrm{M}$ & Total \\
\hline Stars with cold or hot excess & $4 / 11$ & $1 / 4$ & $0 / 3$ & $0 / 2$ & $5 / 20$ \\
Stars with cold excess & $4 / 7$ & $1 / 4$ & $0 / 3$ & $0 / 2$ & $5 / 16$ \\
Stars with cold excess only & $3 / 4$ & $1 / 2$ & $0 / 2$ & $0 / 1$ & $4 / 9$ \\
Stars with hot excess & $1 / 7$ & $0 / 2$ & $0 / 1$ & $0 / 1$ & $1 / 11$ \\
Stars with hot excess only & $0 / 4$ & None & None & None & $0 / 4$ \\
Stars with no known excess & None & $0 / 9$ & $0 / 6$ & $0 / 5$ & $0 / 20$ \\
\hline Overall sample & $4 / 11$ & $1 / 13$ & $0 / 9$ & $0 / 7$ & $5 / 40$ \\
\hline
\end{tabular}

Notes. A cold excess corresponds to a FIR excess detected anywhere between $70 \mu \mathrm{m}$ and $160 \mu \mathrm{m}$ with Spitzer and/or Herschel (same as Table 2). A hot excess corresponds to an excess reported in the NIR around $2.2 \mu \mathrm{m}$ (Absil et al. 2009, 2013).

zodis, significantly higher than the value measured for Group 1 stars, which is 15 zodis. The distributions measured for the two groups of stars are then significantly different, pointing to a positive correlation between the measured KIN MIR excess and the presence of a previously known infrared excess. In fact, and as shown in Table 3, most of these infrared excess stars (16 out of 20) have a FIR excess, and it is thus really the presence of a cold FIR excess that is the strongest indicator of a MIR excess. Out of the 16 stars with a known FIR excess, 5 show a KIN excess. In comparison, of the 24 stars that have no cold excess, none show a KIN excess. A Fisher's exact test shows that there is only a $0.7 \%$ chance of detecting rates as discrepant if the two groups had the same distribution. This apparent correlation of warm (zodi-like) and cold (Kuiper-like) infrared excesses is explored more quantitatively in Section 4.6.

Figure 5 further examines the possible correlation of the KIN measurements with specific types of infrared excess (or lack of it). It shows the KIN excess rates derived for six different subgroups from the original sample: stars with previously known excesses (cold or hot, 20 of them), cold excess (16), cold excess only (9), hot excess (11), hot excess only (4), and stars with no infrared excess reported (20). For each subgroup, the KIN excess rate in the $8-9 \mu \mathrm{m}$ bin is defined as the fraction of stars showing an excess significance larger than $3 \sigma$. For the sake of this analysis, the KIN excess rate in the $8-13 \mu \mathrm{m}$ region is defined as the fraction of stars showing a correlated excess significance greater than $2 \sigma$, which includes six bona fide excess stars and two KIN excess candidates (Altair and Vega; see Section 5.2). The (asymmetric) statistical uncertainty on the excess rate results from a numerical integration of the binomial distribution. As described in the Appendix of Burgasser et al. (2003), the excess rate lower and upper uncertainty limits are computed so that the integrated probability to fall between these limits is $68.2 \%(34.1 \%$ on each side of the observed excess rate), equivalent to the confidence level of $\pm 1 \sigma$ Gaussian limits. The largest KIN excess fraction is found around stars with a previously known cold excess: between 5/16 and $7 / 16$ depending on the wavelength range and detection threshold used. In comparison, a smaller fraction of stars with a hot excess show a MIR excess: between 1/11 and 4/11, depending on the wavelength range and detection threshold used. However, most of these hot excess stars have a known cold excess as well. Restricting to stars with a "hot excess only" leaves four stars, and none of them show an excess, to the possible exception of Altair, which shows a candidate KIN excess (see Section 5.2.3).

\subsection{Underlying Zodi Luminosity Distribution}

Given the observed distributions, we attempt here to derive some relevant information about the "true" underlying zodi luminosity distribution of main-sequence stars. There are several difficulties to overcome in order to do so, including possible biases in the sample, finite sampling errors, and measurement noise. We address here these different issues and present the final constraints that can be derived from the KIN measurements.

\subsubsection{Subgroups of Stars}

In order to reduce biases in the overall sample, while still keeping a statistically significant number of stars, we first split it into the two groups defined above and studied the characteristics of each group independently. Group 1 is composed of 20 targets with no previously reported infrared excess of any kind. It contains no A stars and can be essentially seen as a sample of solar-type stars (nine $F$, six $G$, four $K$, and one $\mathrm{M}$ ). It is the relevant group to characterize in preparation for a future exo-Earth direct imaging mission around Sun-like 
stars. ${ }^{18}$ Conversely, Group 2 corresponds to targets with previously reported infrared excesses (mostly cold ones) and is composed of A stars in majority ( $11 \mathrm{~A}, 4 \mathrm{~F}, 3 \mathrm{G}$, and $2 \mathrm{~K}$ ). Since $\eta \mathrm{Crv}$ is a clear outlier and a very rare case $\left(\simeq 1\right.$ in $10^{4}$ according to Kennedy \& Wyatt 2013) of a $>1$ Gyr old F star with a large $\mathrm{N}$-band excess, we removed it from all further subgroup analyses. While interesting to study for comparison, Group 2 is admittedly more heterogeneous than Group 1, and we further divided it into two more uniform subgroups: Group 2a, composed of 15 stars with a FIR (cold) excess, and Group 2b, which is further restricted to the eight solar-type (FGK) stars with a FIR excess. Comparing the properties of Groups 1, 2a, and 2b should help further assess whether the existence of a cold dust reservoir is a key factor, regardless of spectral type.

\subsubsection{Maximum Likelihood Estimations of the Underlying Zodi Luminosity Function}

One simple way to analyze the data is to assume some representative underlying zodi distributions that are fully characterized by one or two parameters. We tested three kinds of distributions: uniform, truncated Gaussian, and lognormal, and estimated their parameters (denoted $\boldsymbol{\theta}$ ) using a maximum likelihood estimation (MLE) method. More precisely, if the assumed zodi luminosity function is $p(z)$, and each star group contains a set of $N$ independent identically distributed observations $\left(z_{i}\right.$, $\left.\sigma_{i}\right)_{1 \leqslant i \leqslant N}$, the likelihood estimator of parameters $\boldsymbol{\theta}$ given the data is computed as

$$
L\left(z_{1}, z_{2}, \ldots, z_{N} \mid \boldsymbol{\theta}\right)=\prod_{i=1}^{N} p_{\text {noisy }}\left(z_{i}, \boldsymbol{\theta}\right),
$$

with

$$
p_{\text {noisy }}\left(z_{i}, \boldsymbol{\theta}\right)=\left[p(z) * \operatorname{Gaussian}\left(0, \sigma_{i}\right)\right]_{\left(z_{i}\right)},
$$

and where the $*$ sign designs a convolution operation. Indeed, for each star, the observed zodi value is affected by a random measurement error, represented by a zero mean Gaussian noise with known standard deviation $\sigma_{i}$, and this corresponds to a convolution operation in distribution space. For each zodi distribution assumed, the distribution parameters are determined by maximizing the likelihood estimator of Equation (15). In order to estimate confidence intervals on these parameters, we use regular maximum likelihood estimator properties described in Appendix B. For correct interpretation of these MLE results, it is important to recognize that while the MLE distribution fits the data best, a range of distributions are also compatible with the data, and this uncertainty is captured in the likelihood curves of the distribution parameter(s).

Figure 6 shows the results obtained when assuming a uniform zodi-level distribution extending from zero to some maximum value $Z_{\max }$, the only distribution parameter in this simple case. The upper panels show the likelihood curves and maximum likelihood parameters derived for the four different groups of stars considered. The parameter $68 \%, 90 \%$, and $98 \%$ confidence intervals (respectively $84 \%, 95 \%$, and $99 \%$ for one-sided lower or upper bounds) are indicated by the intersection of the likelihood curves with the three corresponding horizontal lines. The maximum likelihood distribution and resulting fraction of stars above a given zodi level are shown in the middle and lower panels of Figure 6. In the uniform zodi model case, the

\footnotetext{
18 Especially given that such a mission will likely avoid any rare solar-type star with a previously known bright FIR excess.
}

maximum likelihood zodi distribution is significantly narrower for stars with no previously known excess (Group 1) than for any other group, and this result still holds when taking distribution estimation uncertainties into account. At the $99 \%$ confidence level, the median zodi value $\left(Z_{\max } / 2\right)$ is below 75 zodis for Group 1 stars, while it is above 130 zodis for stars with a cold excess (Group 2a) and above 85 zodis for FGK stars with a cold excess (Group 2b). Because Group 2b contains only eight stars, its zodi distribution extent and parameters are generally not as accurately constrained as for the other groups, which can be readily seen in the likelihood curves.

Figures 7 and 8 show the results of similar analyses conducted assuming more realistic zodi luminosity functions: a truncated (positive) Gaussian and a lognormal zodi distribution, respectively. Both types of distributions provide very similar results with the data MLE analysis: the zodi distribution of Group 1 stars is found to have the smallest extent, peaking toward the lowest zodi values, while fairly identical quasi-Gaussian distributions are found for stars in Groups 2 and $2 \mathrm{a}$, centered around a level of 200 zodis (consistent with the findings of Section 4.2). Group $2 \mathrm{~b}$ mean zodi levels are less accurately constrained but are found to be fairly similar to those of Groups 2 and 2a, although the distribution derived for Group $2 b$ appears wider, with a larger fraction of stars at low zodi levels.

The overall results of these maximum likelihood analyses are summarized in Table 4, which indicates the median zodi values derived for each group and for the different types of distributions assumed. For solar-type stars with no excess previously detected, the derived maximum likelihood median values are consistently below 20 zodis. In the case where the underlying distribution is represented by a lognormal distribution, we further find that the median zodi level for this group is lower than 60 solar zodis with $95 \%$ confidence and lower than 90 solar zodis with $99 \%$ confidence. For stars with a known reservoir of distant cold dust (Group 2a), we find a most likely median zodi level of about 200 zodis, and above 140 zodis with $95 \%$ confidence. This is a factor of a few below the detection limit of the instrument, but still significantly higher than for Group 1 stars. There is then a statistically significant correlation between the existence of a cold FIR excess and the strength of the $10 \mu \mathrm{m}$ excess. Interestingly, this correlation still holds when excluding A stars and only keeping solar-type stars (Group 2b), in which case the median zodi value is still above 60 solar zodis with $95 \%$ confidence. Another interesting feature is that even when taking into account the uncertainty on the derived distribution parameters, many of the stars with a cold excess apparently have MIR excesses clustering between $\simeq 100$ and $\simeq 400$ solar zodis, right below the detection limit of the instrument.

\subsubsection{Estimating the " $N$-sample" Zodi Luminosity Function}

Finally, we explored the possibility that the underlying zodi luminosity distribution might be estimated "blindly," i.e., without any a priori assumption on its shape. For each group of stars considered, there are $N$ zodi-level measurements and associated uncertainties (Table 2), referred to hereafter as $\left(z_{i}, \sigma_{i}\right)_{1 \leqslant i \leqslant N}$. Given these observations, assuming that the measurement noise is a zero mean Gaussian and using Monte Carlo simulations, it is possible to estimate the zodi luminosity function of the $N$-sample. The approach we used to do so is based on an iterative blind deconvolution method (B. Mennesson, in preparation). As a first iteration, we consider a uniform distribution ranging from 0 to some maximum zodi level larger 

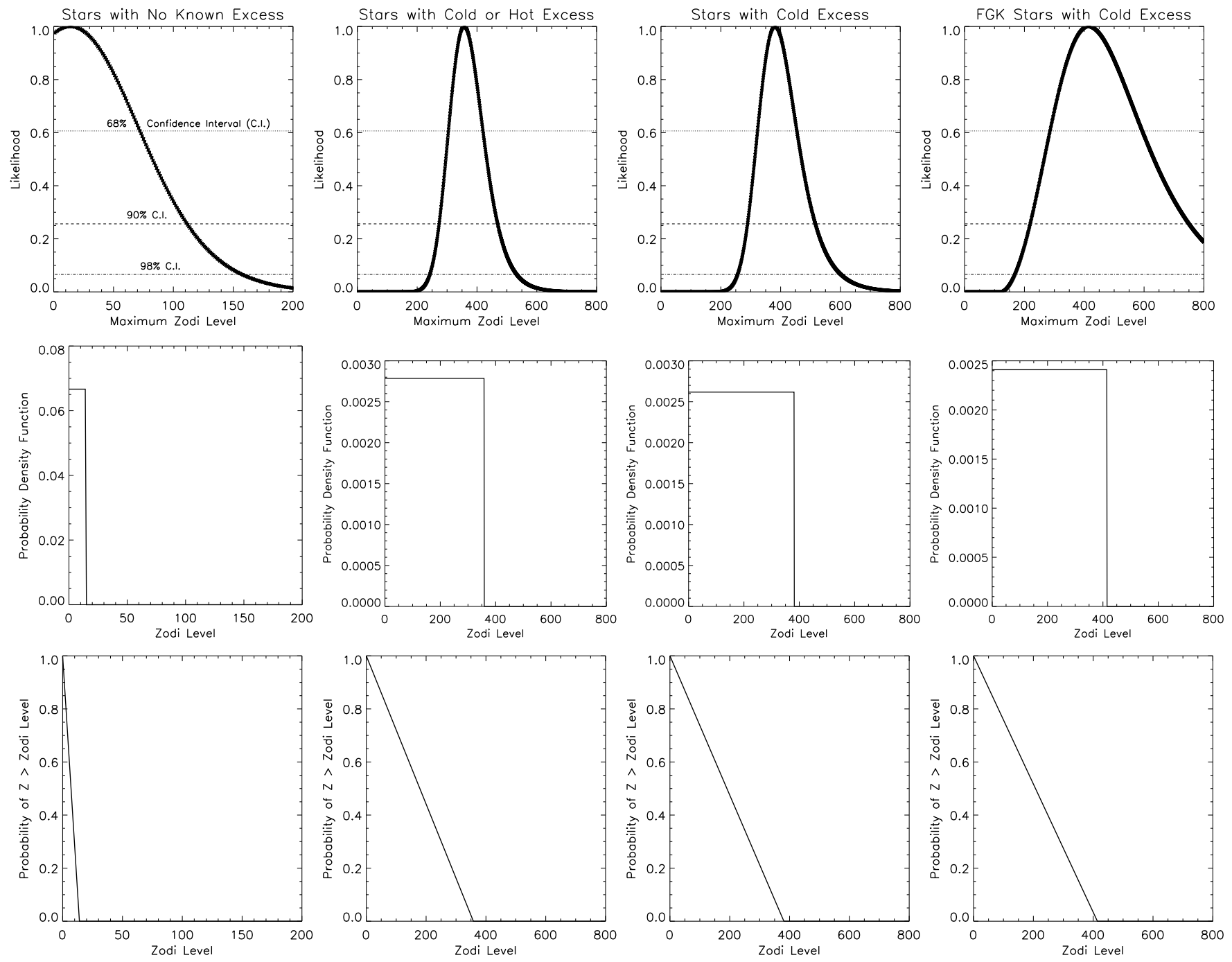

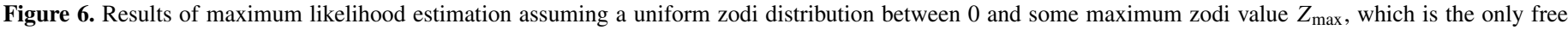

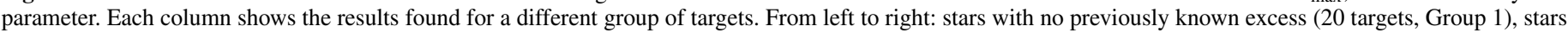

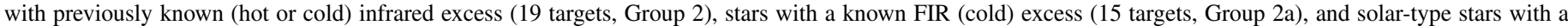

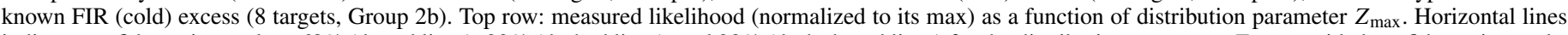

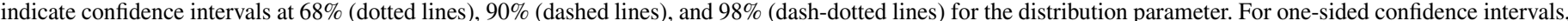

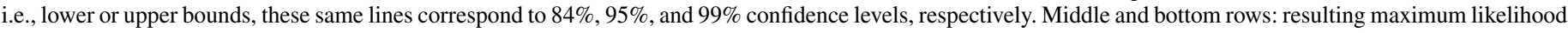
distributions and cumulative fractions given the data. Note the smaller range of zodi levels displayed in the first column panels.

than any of the observed values (say, 10,000 solar zodis). We denote by $p_{1}(z)$ this initial guess distribution and by $P_{1}(z)$ the corresponding fraction of stars with zodi levels above a certain level z. A large number (e.g., $N_{\mathrm{mc}}=10^{6}$ ) of zodi values are then randomly drawn from it, and for each star observed with measurement uncertainty $\sigma_{i}, N_{\mathrm{mc}}$ "measured" zodi values are simulated. Among the values falling within a small interval centered around $z_{i}$, only a fraction have nominal (no noise) zodi values larger than $z$. Denoting this fraction $P_{2}\left(z \mid z_{i}\right)$, repeating and averaging over the $N$ stars yields $P_{2}(z)=\sum_{i=1}^{N} P_{2}\left(z \mid z_{i}\right) / N$, the second iteration (and better) estimate of the underlying cumulative zodi-level distribution given the data. Iterating on this procedure converges to a final estimate of the $N$-sample zodi luminosity function and its cumulative fraction, as represented in Figure 9 for each of the four groups of KIN targets. While the four distributions found are very dissimilar and likely reflect some real physical differences between the different groups, the best that can be retrieved using this procedure is the luminosity function of the $N$-sample, and it remains challenging to draw from it any quantitative information about the underlying (infinite sample) zodi distribution, for the reasons detailed in the next section.

\subsubsection{Finite Sampling and Measurement Uncertainties}

Finite sampling effects are well understood and described by Bernoulli statistics. Let us assume that the underlying (infinite) population has a cumulated probability $P(z)$ that a star zodi level be above an arbitrary threshold value $z$. If $N$ stars are randomly drawn from that stellar population and perfect (noiseless) measurements are made of their zodi levels, the empirical fraction $P_{N}(z)$ of this $N$-sample provides an unbiased estimate of $P(z)$ (i.e., converging toward it for large values of $N)$, with a $1 \sigma$ spread equal to $\sqrt{P *(1-P) / N}$. This shows in particular that the largest measurement uncertainty is found for $P=0.5$, which corresponds to the median zodi value of the distribution. For $N=20$, for instance, this means that even with a perfect instrument, $P(z)$ cannot be measured with a $1 \sigma$ uncertainty lower than $11.2 \%$ at the median zodi 

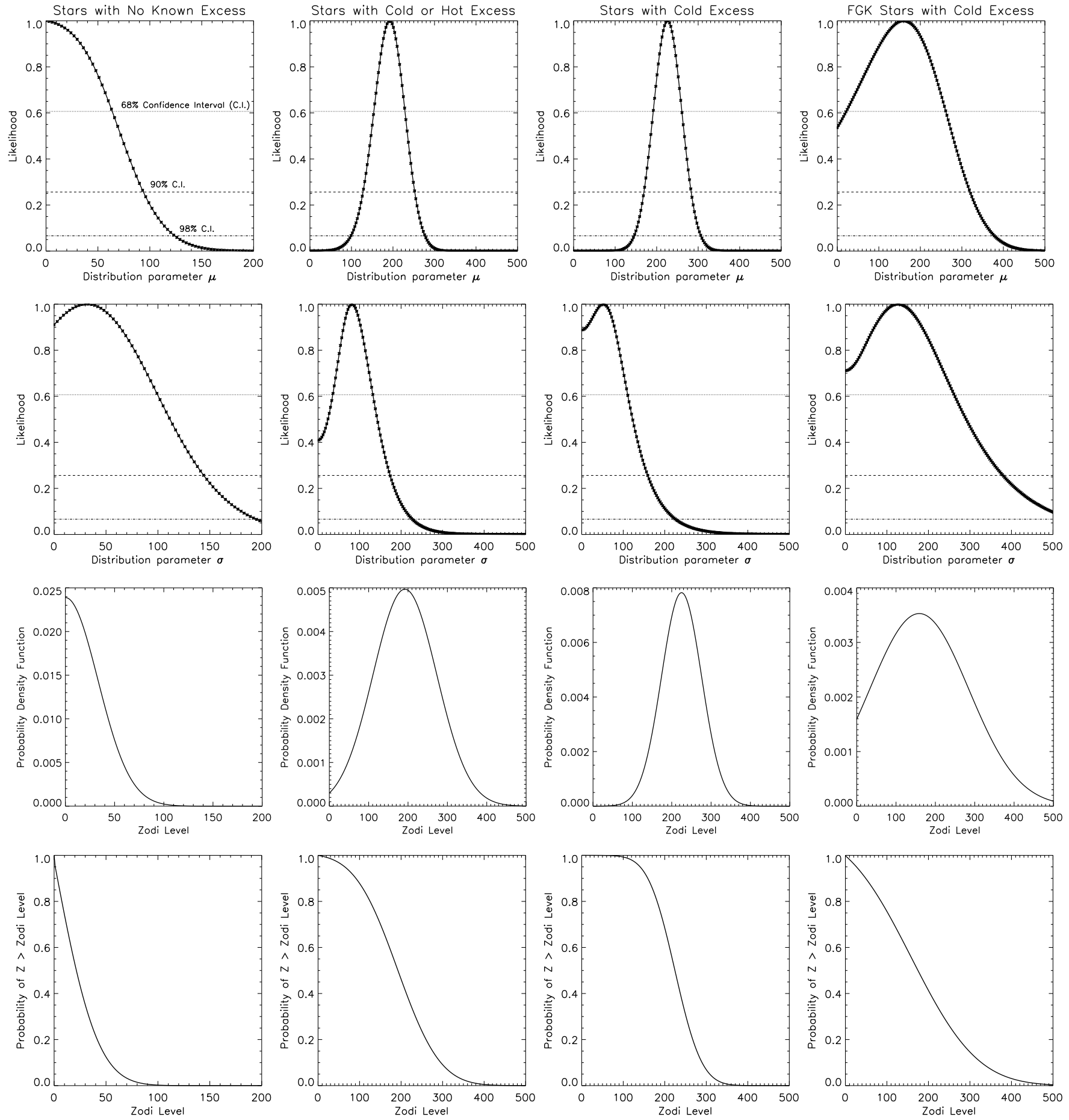

Figure 7. Results of maximum likelihood estimation assuming a truncated Gaussian zodi distribution with parameters $\mu$ and $\sigma$, respectively, the mean and standard deviation of the parent Gaussian distribution. Each column shows the results found for a different group of targets, as defined in Figure 6 and indicated at the top. Top two rows: measured likelihood (normalized to its max) as a function of distribution parameter values. Horizontal lines indicate confidence intervals at $68 \%$ (dotted lines), $90 \%$ (dashed lines), and 98\% (dot-dashed lines) for the distribution parameter. For one-sided confidence intervals, i.e., lower or upper bounds, these same lines correspond to $84 \%, 95 \%$, and $99 \%$ confidence levels, respectively. Bottom two rows: resulting maximum likelihood distributions and cumulative fractions. Note the smaller range of zodi levels displayed in the first column panels.

level of the distribution, whatever its value is. ${ }^{19}$ It also shows that the $1 \sigma$ offset between the $N$-sample distribution and the true distribution depends on the distribution itself (via $P(z)$ ).

19 Conversely, as the value of $P$ gets close to 0 or 1, the uncertainty diminishes, although the actual distribution of measured values becomes highly skewed and the $1 \sigma$ spread is no longer a good metric to use for error bars.
In other words, even for perfect measurements, the retrieved $\mathrm{N}$-sample distribution of Figure 9 generally differs from the true distribution, and the offset between the two, which sets the estimation uncertainty, is difficult to quantify.

Additionally, the observed zodi levels are affected by the KIN measurement error, which is 200 zodis per star on average, meaning that the $N$-sample distribution itself is not perfectly 

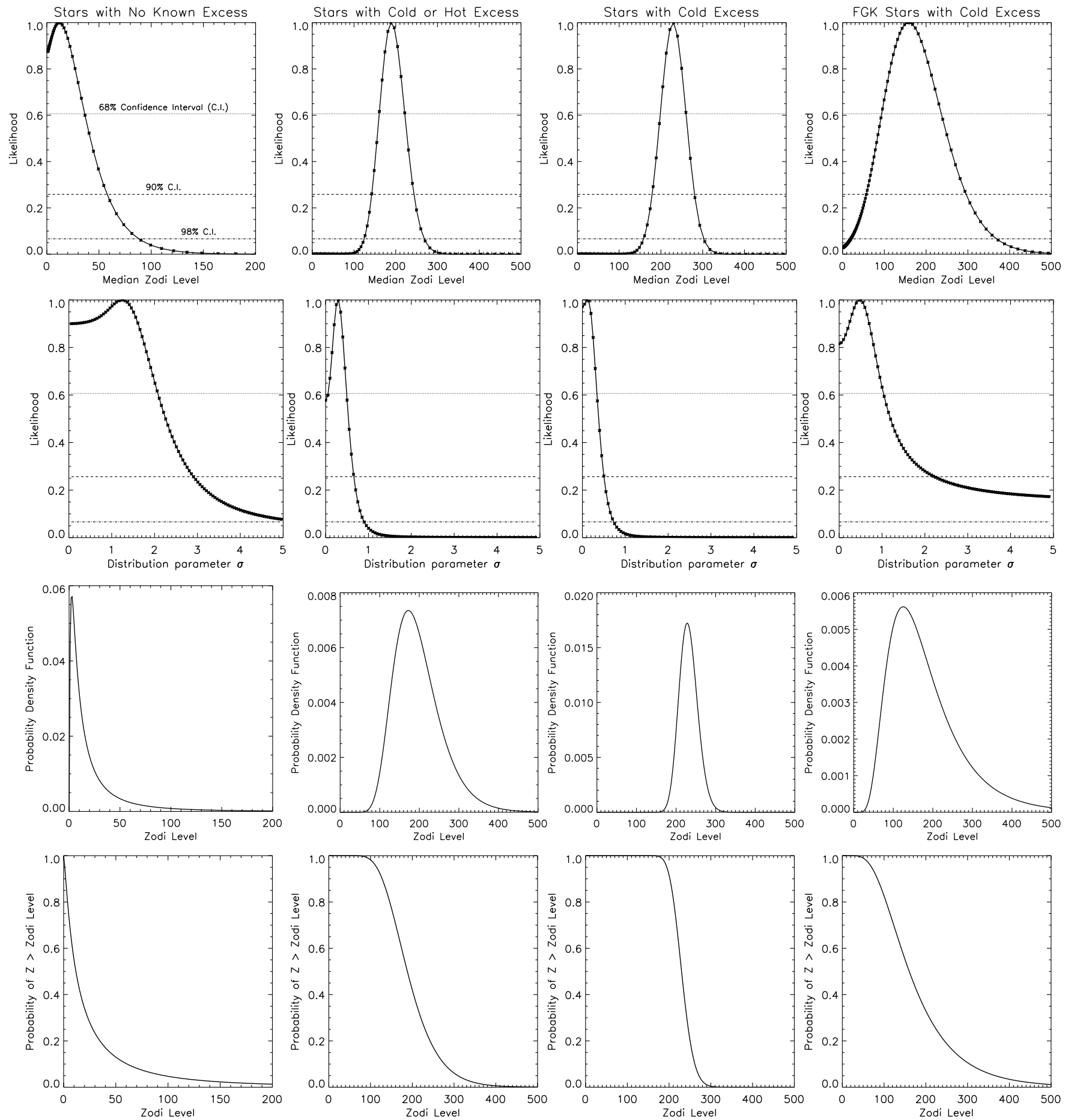

Figure 8. Results of maximum likelihood estimation assuming a lognormal zodi distribution with parameters $\mu$ and $\sigma$. Each column shows the results found for a different group of targets, as defined in Figure 6 and indicated at the top. Top two rows: measured likelihood (normalized to its max) as a function of distribution parameter values. The top row is actually plotted as a function of the distribution median value, which is the exponential of the $\mu$ parameter. Horizontal lines indicate confidence intervals at 68\% (dotted lines), $90 \%$ (dashed lines), and 98\% (dot-dashed lines) for the distribution parameter. For one-sided confidence intervals, i.e., lower or upper bounds, these same lines correspond to $84 \%, 95 \%$, and $99 \%$ confidence levels, respectively. Bottom two rows: resulting maximum likelihood distributions and cumulative fractions. Note the smaller range of zodi levels displayed in the first column panels.

measured. Numerical simulations-and common sense-show that if $N$ is the number of measurements, then no information can be reliably retrieved over zodi intervals much smaller than $\simeq 200 / \sqrt{N}$. It also means that if the underlying distribution is significantly narrower than this limit, its actual shape cannot be derived accurately by a blind method. We verified this numerically by randomly drawing $N$ zodi levels from a "perfectly known" exozodiacal luminosity distribution, adding Gaussian measurement noise with a $1 \sigma$ error of 200 zodis and applying the blind deconvolution method described above to the resulting set of observed values. The algorithm converged to a single solution in all cases simulated, but it only provided correct (unbiased) estimates of the drawn distribution when the parent distribution was broader than $\simeq 200 / \sqrt{N}$, which corresponds to a width of about 50 zodis in the case of 20 stars. In order to derive information on the shape of such a narrow distribution, some a 

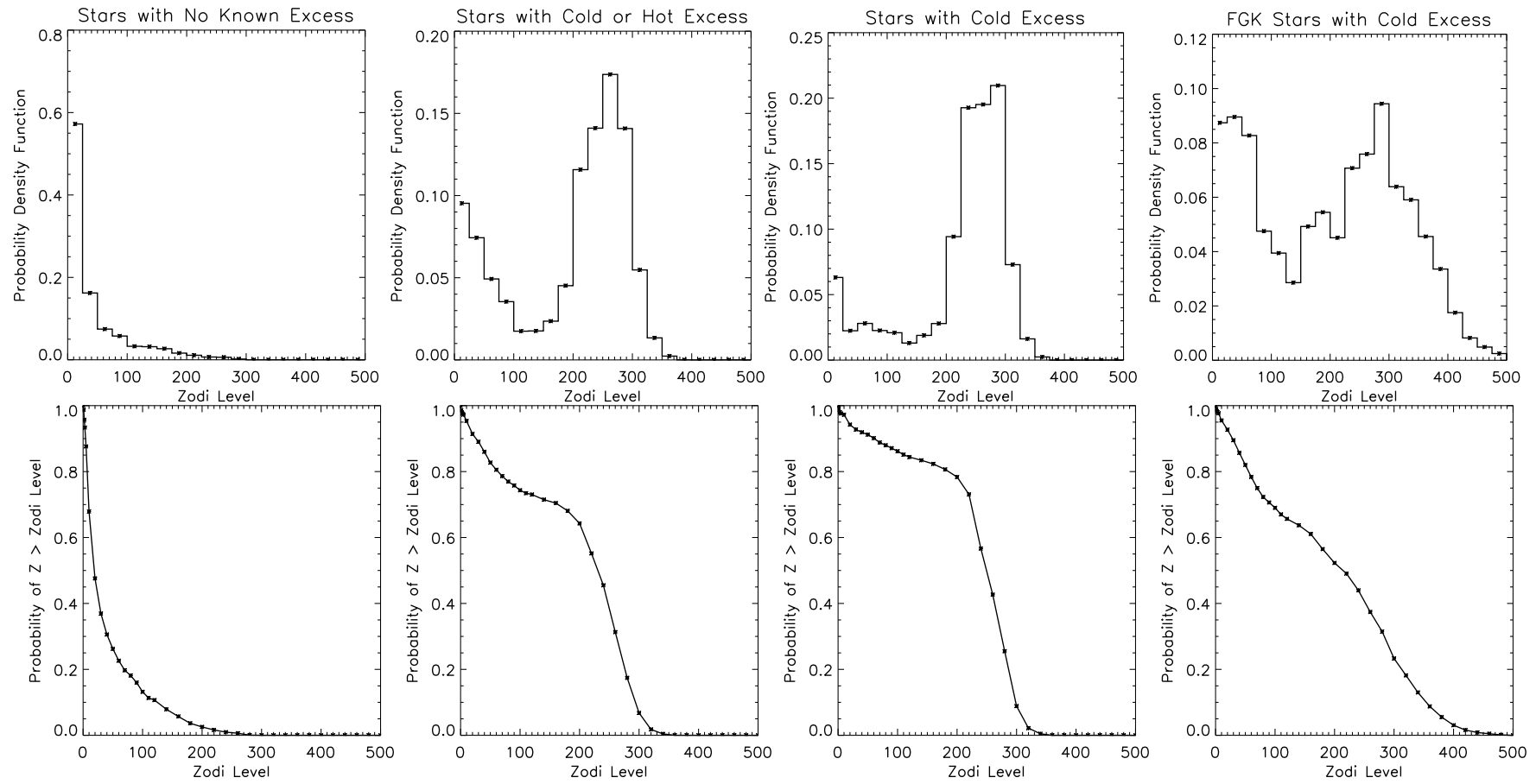

Figure 9. Zodi-level distributions and cumulative fractions derived for different subgroups of stars, using the iterative blind deconvolution method. Histogram bin size is 25 zodis. Each column shows the results found for a different group of targets, as defined in Figure 6 and indicated at the top. From left to right: stars with no previously known excess (20 targets, Group 1), stars with previously known (hot or cold) infrared excess (19 targets, Group 2), 15 stars with a known FIR (cold) excess (15 targets, Group 2a), and solar-type stars with a known FIR (cold) excess ( 8 targets, Group $2 \mathrm{~b}$ ). Note the widely different distributions derived for stars with a cold excess vs. stars with no previously known excess.

Table 4

Median Zodi Levels

\begin{tabular}{lcccc}
\hline \hline Distribution Type & Group 1 & Group 2 & Group 2a & Group 2b \\
& Stars with No Known Excess & Stars with Cold or Hot Excess & Stars with Cold Excess & FGK Stars with Cold Excess \\
\hline Uniform & $7[0-56]$ & $177[137-234]$ & $190[145-259]$ & $207[110-375]$ \\
Truncated Gaussian & 20 & 190 & 227 & 160 \\
Lognormal & $12[0-60]$ & $190[142-242]$ & $230[178-280]$ & $160[60-296]$ \\
From blind deconvolution & 18 & 240 & 250 & 220 \\
\hline
\end{tabular}

Notes. Median zodi values derived for four different subgroups of stars observed by the KIN assuming different underlying distributions. Group 1: 20 solar-type stars with no previously known IR excess of any kind; Group 2: 19 stars with previously known infrared excess (hot or cold); Group 2a: 15 stars with a known FIR (cold) excess. Group 2b: 8 FGK stars with a known FIR (cold) excess. In all cases, the most likely median zodi value is quoted, together with its $90 \%$ confidence interval when available. One-sided bounds have a 95\% confidence level, meaning that in the lognormal case, for instance, and with 95\% confidence, the Group 1 median zodi level is smaller than 60 solar zodis while that of Group 2a is higher than 178 solar zodis.

priori assumption must be made on the nature of the distribution (e.g., Gaussian or lognormal) so that only one or two ensemble parameters need to be derived down to the $\simeq 50$ zodi accuracy level (e.g., the mean or median). For zodi luminosity distributions derived using the blind deconvolution method (Figure 9), we used a bin size of 25 zodis. This value provides adequate sampling given the smallest structures that can be reasonably identified given our measurement uncertainties.

\subsubsection{Summary of Zodi-level Distribution Modeling Results}

Because of the finite sampling and measurement uncertainty effects described in the previous section, we regard the results of the blind deconvolution method as more qualitative than the MLE results, especially for solar-type stars with no previous excess (Group 1), which seem to exhibit a narrow distribution skewed toward low zodi values. For such stars, we favor the results obtained assuming a truncated Gaussian (Figure 7) or a lognormal (Figure 8) distribution, which are summarized in Table 4. We note, however, that many of the distribution characteristics derived by the blind deconvolution method are consistent with the results found using the maximum likelihood estimations in the lognormal and truncated Gaussian cases. For Groups 2 and $2 \mathrm{a}$, the derived zodi distributions are similar and show a large fraction of stars with zodi levels around 200-300 zodis. Since the blind deconvolution method does not assume any simple a priori shape, its derived distributions present additional complex features. A compelling one is the double peak distribution found for Group $2 b$ (Figure 9), which suggests that solar stars with FIR excess previously detected can also have low levels of MIR zodi emission. In other words, while all stars with a detected KIN MIR emission have a large cold FIR excess, the reciprocal may not be true. The tail found at low zodi values for Group 2 stars may also be real and is much attenuated for Group 2a stars. This reflects the fact that stars with a hot excess but no cold excess (only present in Group 2) have statistically lower zodi levels than those with cold excess 
detected. This difference between the distributions derived for Groups 2 and 2a at low zodi levels is also seen in Figures 7 and 8 .

\subsection{Discussion}

\subsubsection{Warm Exozodi Dust and Cold Outer Dust Reservoir}

While there is strong apparent correlation of the KIN results with spectral type (and possibly age), because no main-sequence star later than F2 shows a MIR excess, another key parameter is then the presence of cold dust in the system. From the sample of 40 stars surveyed, it appears that if a star has a cold FIR excess, it has a higher probability of showing a $10 \mu \mathrm{m}$ excess strong enough to be detected by the KIN. Reciprocally, and maybe more interestingly, all five stars with a formally identified KIN excess had a previously known FIR excess attributed to a cold reservoir of dust and cometary material. In other words, large amounts of distant cold dust seem to be required for there to be much warmer exozodi emission detectable by the KIN around $10 \mu \mathrm{m}$, which necessarily originates from within the first few AUs. The statistical analysis presented in the previous section further establishes and quantifies this connection. It shows that over a wide range of reasonable assumptions for the shape of the underlying zodi luminosity function, stars with a cold excess (regardless of spectral type) have a median zodi value above $\simeq 140$ zodis with $95 \%$ confidence. This is in stark contrast to the case of stars with no cold dust reservoir, for which the median zodi level is found below 60 zodis with $95 \%$ confidence under the same assumptions. As shown in Section 4.6 and Table 4, the correlation between warm (zodi-like) and cold (Kuiper-like) dust is also observed when concentrating on solartype stars: FGK stars with a cold excess (Group 2b) have a statistically larger exozodi level than those with no cold excess (Group 1). Although this particular result would benefit from observations of a larger sample, such stars are then a priori bad targets for future direct imaging missions searching for Earthlike planets around Sun-like stars. While warm dust detected by the KIN necessarily resides within a few AU from the host star, its correlation with the presence of cold dust suggests that it finds its origin in the outer regions of the system. This is in contrast to an "in situ" scenario where warm dust would be formed locally by collisions of parent bodies located in the inner few AUs, in which case only a MIR excess is detected, as observed around very few main-sequence solar-type stars, such as HD 69830 (Beichman et al. 2005, 2006b), BD +20307 (Weinberger et al. 2011), and HD 15407A (Fujiwara et al. 2012). A popular mechanism for feeding dust to the inner system is the steadystate cometary delivery scenario (e.g., Wyatt et al. 2007; Bonsor et al. 2014). In that case, dust and cometary material from a more distant planetesimal belt is being scattered inward because of dynamical perturbations (Nesvorny et al. 2010; Bonsor et al. 2012; Bonsor \& Wyatt 2012). This would resemble the "falling evaporating bodies" phenomenon observed in the $\beta$ Pic inner disk (Beust \& Valiron 2007). For systems with a particularly high level of excess, these could be experiencing an elevated level of cometary activity due to a recent major dynamical instability such as the late heavy bombardment that happened early in the history of our own planetary system (Gomes et al. 2005).

However, another important result from the KIN observations of stars with FIR excesses, even considering the uncertainty on the shape of the zodi distribution and the derived maximum likelihood parameters, is that a large fraction of these stars appear to have zodi levels between 100 and 400 zodis, i.e., just below the KIN detection threshold. This result provides important constraints on the physical mechanism causing the emission. Once again, it appears to rule out transient phenomena such as recent local collisions that would be expected to result in a sudden brightening followed by a slow decay leading to a distribution with more faint excesses than bright ones (Kennedy \& Wyatt 2013), and the inferred distribution is more compatible with the steady passing of comets from the outer belt into the inner regions. However, it is unclear why there would be a common zodi level for all stars, unless this is related somehow to the sample selection, e.g., if the ratio of zodi to cold dust is constant and the sample has uniform cold dust properties. Since the sample cold dust levels are not identical and the cometary flux depends on the scattering properties of the underlying planetary system (Bonsor et al. 2012), which is likely to show some diversity, this cannot be the sole explanation. One possibility could be that a common level arises from the physics of dust production; e.g., the balance of collisions and Poynting-Robertson (P-R) drag means that dust levels close to the star in this regime are independent of many of the properties of the outer belts (Wyatt 2005; van Lieshout et al. 2014). Indeed, such a model predicts that the brightest outer disks all converge to similar optical depth in the inner disk, i.e., the same MIR brightness, only with small variation versus spectral type. Figure 10 shows the $8.5 \mu \mathrm{m}$ disk fractional flux expected as a function of the temperature of the outer cold dust using the simplified approach developed by Wyatt (2005). In this model, dust produced in the outer belt is of single size, and the inner dust spatial distribution and its MIR emission are solely governed by the balance between grain collisions and P-R drag, with an assumed $\beta$ parameter of 0.5 . In this case, all grains have the size of the smallest particles that can remain bound to the star. Assuming a Mie scattering coefficient $Q_{\mathrm{PR}}$ of 1 (which is strictly valid for grains $\gg 1 \mu \mathrm{m}$ ), this particle size can be directly derived from the stellar luminosity and mass and from the grain density (see Equation (6) of Wyatt et al. (2007)). Assuming silicate dust grains, we get, for instance, a grain size of $15 \mu \mathrm{m}$ around Vega (A0V star) and $2 \mu \mathrm{m}$ around 10 Tau (F8V). This gives an idea of the range of dust grain sizes expected from this model for most targets in our cold excess sample (11 A and F stars out of 16). For lower-mass stars, the simple analytical relation from Wyatt et al. (2007) is no longer valid, and $Q_{\mathrm{PR}}<1$ to such an extent that it is not always possible to blow particles out, e.g., as shown by Sheret et al. (2004) in the case of $\epsilon$ Eri. Grains around these stars might then be very tiny, i.e., smaller than a micron. The displayed model curves are computed for a cold disk optical depth of $10^{-4}$, but any value above $\simeq 10^{-5}$ would predict similar MIR fractional fluxes. The KIN measurements for the 16 stars with a cold outer dust belt are shown for comparison. In order to convert the observed KIN excesses into a physical excess, a zodi brightness distribution must be assumed, and we adopted a solar-system-like distribution to do so (Kelsall et al. 1998, together with the Zodipic model). For the cold dust temperature, we used the values derived from Spitzer and Herschel excess measurements at 24, 70, 100, and $160 \mu \mathrm{m}$, assuming a single dust belt radius. These temperatures are listed in Table 2, together with appropriate references to MIR/FIR excess observations for each star. Despite the simplicity of this model, in particular its assumption of single-sized dust grains, and although the dependence on the outer disk temperature appears even weaker than expected, the KIN measurements are 


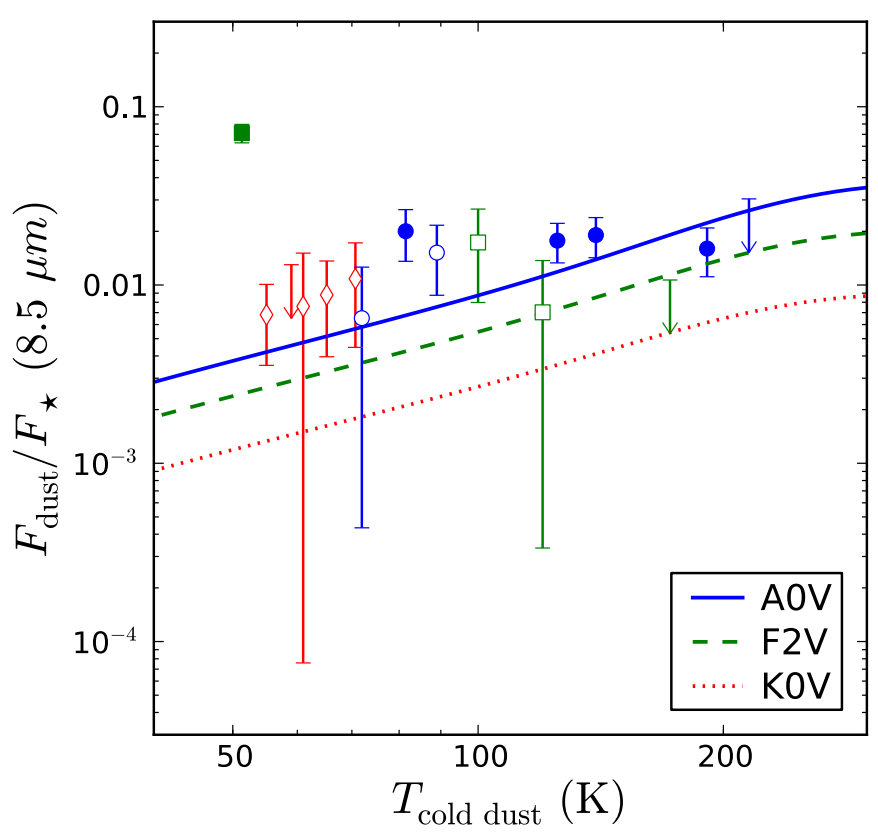

Figure 10. KIN measurements of MIR relative disk flux ratio and comparison with a P-R drag/collisions model. Data are for 16 targets with cold dust previously detected in the FIR (15 stars of Group 2a, plus the top left outlier $\eta$ Crv). Circles: A stars; squares: F stars; diamonds: G and K stars. Stars with a detected KIN excess are indicated with filled symbols. Error bars are $\pm 1 \sigma$, except for "negative excess flux measurements," which are given as $3 \sigma$ upper limits and indicated by downward-pointing arrows (A star: $\delta$ Uma; F star: $\eta$ Lep; and G star: $\tau$ Ceti). The three model curves show the fractional excess expected for stars of different spectral types (solid line: A0V; dashed line: F2V; dotted line: $\mathrm{K} 0 \mathrm{~V}$ ) assuming that a cold outer belt feeds dust to the inner system through the joint effect of P-R drag and grain collisions (Wyatt 2005). In this model, the MIR disk flux depends only weakly on the optical depth of the outer disk, as long as it is higher than $\simeq 10^{-5}$, which is the case of all stars considered here An optical depth of $10^{-4}$ is assumed for these plots. Most disk fractional fluxes concentrate between $\simeq 0.5 \%$ and $2 \%$. Observed fluxes are higher than predicted by the model for $\mathrm{G} / \mathrm{K}$ stars, but only at the $2 \sigma$ level (error bars are highly asymmetric because of the log scale); see Section 4.7.1 for further details.

(A color version of this figure is available in the online journal.)

reasonably consistent with the model predictions. In particular, the higher fractional flux level observed around A stars is predicted by the model, as well as the clustering of MIR excesses around $F_{d} / F^{*}=1 \%$ or slightly less, close to the KIN detection limit. A clear outlier is again $\eta \mathrm{Crv}$, whose warm dust is not believed to be generated by P-R drag (Wyatt et al. 2007). While the agreement with this mechanism is reasonable, it is clear that silicate emission features and many other systemspecific aspects, e.g., planetary configurations (Moro-Martin et al. 2005), may also change this simple picture. Nevertheless, the close agreement of the model with the observations shows that the MIR excesses observed by the KIN plausibly have their origin in P-R drag, which in turn implies that the majority of systems with FIR excesses may have MIR excesses at levels not far below the KIN detection threshold.

A possible way to further discriminate between a cometary delivery mechanism (which the presence of massive inner planets may facilitate) and this P-R drag dust transfer scenario (possibly inhibited by such planets) would be to correlate the observed KIN excess with the presence and detailed characteristics of planetary systems around the KIN sample stars. Radial velocity (RV) exoplanetary surveys have examined 20 of the KIN targets (e.g., Fischer et al. 2014). They identified planetary candidates around eight of them, revealing a wide range of system configurations with short-period hot Jupiters ( $\tau$ Boo, $v$ And), Jovian planets in wider orbits (47 UMa, $v$ And, $\kappa$ Crb, and possibly $\epsilon$ Eri), Saturn-mass planets (61 Vir), together with some indication of multiple rocky planets ( $\tau$ Ceti, Tuomi et al. (2013)) and long-term RV trends. While none of these eight FGK stars with RV planets show a bona fide KIN excess, this only shows a lack of correlation with a large MIR excess at $\simeq 500-1000$ times the solar level. A detailed statistical study (similar to Section 4.6) of the correlation of the measured KIN zodi level with planetary characteristics would be required to draw conclusions at lower zodi levels. However, such an analysis is difficult given the observed diversity of system configurations and the limited number of targets showing common planetary features, and it is left for potential future work.

\subsubsection{Hot Dust Phenomenon}

Interestingly, a MIR excess is only detected around A stars with a cold reservoir of dust ( 7 out of the 11 A stars in the sample). This is opposite to the NIR observations of Absil et al. (2013), who found that A stars showed NIR excesses more frequently when they had no FIR excess reported. This could indicate, at least for A stars, that the physical mechanisms responsible for the MIR and NIR excesses are different. In fact, only 1 out of 11 stars with $\mathrm{a} \simeq 1 \%$ NIR excess ( $\beta$ Leo) shows a corresponding KIN excess in the 8-9 $\mu \mathrm{m}$ bin, and it does have a cold FIR excess as well. Restricting the analysis to stars having only a hot excess leaves only four stars (all A type), and none of them show a KIN excess, suggesting again a different physical origin (non-dusty?) for the NIR excess. On the other hand, if the NIR excess emission also originates in dust, it must consist of grains hot enough to contribute a significant flux at $2 \mu \mathrm{m}$ but small enough to have low emissivity at $9 \mu \mathrm{m}$ and remain undetected by the KIN. However, such small grain populations should be rapidly expelled from the system, typically in a few years or so. Their presence around a large fraction of mature stars is then surprising (Absil et al. 2013), as it calls for inordinate dust replenishment rates or poorly understood dust trapping mechanisms and dynamical effects close to the star. The origin of this NIR excess/hot dust phenomenon remains then quite mysterious, and the elucidation of this problem is beyond the scope of this work. In any case, the mechanism responsible for the NIR emission does not seem to produce any significant emission in the MIR, at least down to the KIN detection limit of a few hundred zodis $(1 \sigma)$.

\section{INDIVIDUAL STARS}

From the previous analysis, five stars show a significant $(>3 \sigma)$ excess in either of the two KIN spectral ranges considered, while three more stars appear to be good candidates for an excess.

\subsection{Stars with Detected KIN Excesses}

Overall, five stars show an excess leak detected at $3 \sigma$ or higher in the $8-9 \mu \mathrm{m}$ bin. Only two of them ( $\eta \mathrm{Crv}$ and $\zeta$ Lep) show a broadband excess formally detected as well $\left(S_{\text {cor }}>3\right.$, where $S_{\text {cor }}$ is the correlated excess significance defined in Section 3.6.2), but all five have a broadband excess significance greater than 2 . We give for each star a brief description of the KIN findings in terms of dust location and brightness and summarize previous infrared excess observations. Table 5 summarizes the excess leak values measured as a function of wavelength and spatial frequency for each of these stars, averaging data from different scans and epochs unless strong variations are seen over time. 
Excess Leaks Measured as a Function of Wavelength for the Five Stars Showing an Excess in the 8-9 $\mu \mathrm{m}$ Bin

\begin{tabular}{|c|c|c|c|c|c|c|c|c|c|c|c|c|c|c|}
\hline Star & $\begin{array}{l}\text { Date } \\
\text { (UT) }\end{array}$ & $\begin{array}{c}u \\
(\mathrm{~m})\end{array}$ & $\begin{array}{c}v \\
(\mathrm{~m})\end{array}$ & $\begin{array}{l}x s_{1} \\
(\%)\end{array}$ & $\begin{array}{l}x s_{2} \\
(\%)\end{array}$ & $\begin{array}{l}x s_{3} \\
(\%)\end{array}$ & $\begin{array}{l}x s_{4} \\
(\%)\end{array}$ & $\begin{array}{l}x s_{5} \\
(\%)\end{array}$ & $\begin{array}{l}x s_{6} \\
(\%)\end{array}$ & $\begin{array}{l}x s_{7} \\
(\%)\end{array}$ & $\begin{array}{l}x s_{8} \\
(\%)\end{array}$ & $\begin{array}{l}x s_{9} \\
(\%)\end{array}$ & $\begin{array}{l}x s_{10} \\
(\%)\end{array}$ & $\begin{array}{c}x s_{8-9} \\
(\%)\end{array}$ \\
\hline$\eta \mathrm{Crv}$ & 2008 Apr 17 & 56.31 & 62.34 & $2.80 \pm 0.54$ & $2.36 \pm 0.58$ & $5.26 \pm 0.66$ & $9.27 \pm 0.90$ & $9.48 \pm 1.03$ & $10.40 \pm 1.42$ & $12.01 \pm 1.46$ & $13.60 \pm 2.36$ & $2.82 \pm 4.96$ & $-0.34 \pm 7.58$ & $2.50 \pm 0.41$ \\
\hline$\eta \mathrm{Crv}$ & 2008 May 24 & 46.62 & 51.97 & $2.72 \pm 0.38$ & $4.03 \pm 0.34$ & $5.90 \pm 0.47$ & $8.29 \pm 0.70$ & $8.78 \pm 0.68$ & $9.56 \pm 0.83$ & $10.79 \pm 1.10$ & $10.28 \pm 1.96$ & $8.14 \pm 2.58$ & $11.12 \pm 3.79$ & 3.39 \\
\hline$\eta \mathrm{Crv}$ & 2008 May 24 & 34.39 & 48.37 & $2.33 \pm 0.41$ & $2.63 \pm 0.38$ & $4.72 \pm 0.53$ & $4.02 \pm 0.79$ & $7.71 \pm 0.84$ & $8.45 \pm 1.02$ & $8.58 \pm 1.26$ & $5.68 \pm 1.98$ & $4.21 \pm 3.90$ & $-2.38 \pm 4.07$ & $2.46 \pm 0.31$ \\
\hline$\eta \mathrm{Crv}$ & 2008 May 24 & 25.69 & 46.83 & $2.92 \pm 1.03$ & $1.06 \pm 1.15$ & $5.91 \pm 1.04$ & $1.76 \pm 1.81$ & $2.94 \pm 1.49$ & $4.53 \pm 1.68$ & $7.44 \pm 2.98$ & $-0.31 \pm 4.40$ & $6.08 \pm 8.01$ & $-2.68 \pm 7.94$ & $1.96 \pm 0.92$ \\
\hline$\beta$ Uma & 2008 Маy 26-27 & 26.94 & 76.28 & $0.61 \pm 0.18$ & $0.84 \pm 0.18$ & $0.54 \pm 0.22$ & $0.64 \pm 0.32$ & $0.58 \pm 0.34$ & $0.29 \pm 0.36$ & $0.53 \pm 0.47$ & $0.61 \pm 0.65$ & $1.21 \pm 0.97$ & $0.50 \pm 1.58$ & $0.71 \pm 0.18$ \\
\hline 3 Leo & 2008 Feb 18 & 54.86 & 64.81 & $1.46 \pm 0.46$ & $1.07 \pm 0.24$ & $0.76 \pm 0.28$ & $0.48 \pm 0.30$ & $.47 \pm 0.25$ & $0.09 \pm 0.30$ & $1.02 \pm 0.60$ & $0.80 \pm 0.70$ & $1.97 \pm 0.99$ & $-1.47 \pm 1.70$ & $1.25 \pm 0.33$ \\
\hline$\beta$ Leo & 2008 Apr 16 & 55.63 & 62.74 & $-0.15 \pm 0.28$ & $-0.09 \pm 0.25$ & $-0.47 \pm 0.39$ & $-0.20 \pm 0.30$ & $0.21 \pm 0.31$ & $-0.26 \pm 0.53$ & $-0.14 \pm 0.65$ & $0.51 \pm 0.95$ & $-0.81 \pm 1.36$ & $-0.64 \pm 2.61$ & $-0.12 \pm 0.26$ \\
\hline$\beta$ Leo & 2009 Jan 11 & 52.10 & 55.93 & $0.67 \pm 0.26$ & $0.72 \pm 0.21$ & $0.53 \pm 0.24$ & $0.79 \pm 0.44$ & $0.56 \pm 0.55$ & $-0.20 \pm 0.55$ & $1.73 \pm 0.81$ & $-1.08 \pm 1.43$ & $2.10 \pm 2.38$ & $-4.48 \pm 3.68$ & $0.73 \pm 0.20$ \\
\hline Lep & 2008 & 36.05 & 50.36 & 67 & 41 & 79 & 28 & 15 & 39 & 87 & 12 & 2.58 & .44 & 0.72 \\
\hline$\zeta$ Lep & 2009 Jan 9-13 & 45.65 & 54.44 & $0.37 \pm 0.31$ & $0.64 \pm 0.23$ & $1.15 \pm 0.26$ & $0.78 \pm 0.47$ & $1.46 \pm 0.47$ & $1.35 \pm 0.60$ & $3.19 \pm 0.90$ & $1.81 \pm 1.18$ & $0.34 \pm 1.84$ & $2.31 \pm 3.82$ & $0.55 \pm 0.20$ \\
\hline$\zeta$ Lep & 2011 Feb 14 & 31.49 & 49.33 & $2.20 \pm 1.44$ & $0.68 \pm 1.02$ & $2.37 \pm 1.27$ & $2.61 \pm 1.37$ & $-1.75 \pm 1.72$ & $-0.49 \pm 2.37$ & $-5.94 \pm 3.62$ & $-0.66 \pm 5.11$ & $0.81 \pm 7.39$ & $-7.74 \pm 10.49$ & $1.19 \pm 0.92$ \\
\hline$\gamma \mathrm{Oph}$ & 2008 Jul 16-17 & 43.65 & 64.79 & $0.98 \pm 0.36$ & $0.69 \pm 0.35$ & $0.90 \pm 0.42$ & $1.07 \pm 0.77$ & $1.03 \pm 0.75$ & $2.54 \pm 0.85$ & $3.27 \pm 0.95$ & $3.72 \pm 1.68$ & $5.23 \pm 2.37$ & $3.56 \pm 2.90$ & $0.87 \pm 0.28$ \\
\hline
\end{tabular}

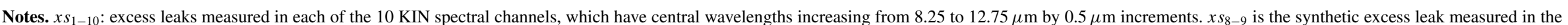
$8-9 \mu \mathrm{m}$ channel. 
Further detailed modeling of individual targets is left for future analysis.

$$
\text { 5.1.1. } \eta \mathrm{Crv}
$$

The most significant KIN excess is detected around $\eta \mathrm{Crv}$, and it has to come from within $\simeq 3 \mathrm{AU}$ (according to the IWA listed in Table 1). This object was previously known to have high levels of circumstellar dust, including detections with Spitzer Multiband Imaging Photometer (MIPS) at $70 \mu \mathrm{m}$ (Beichman et al. 2006b), Spitzer infrared spectography (IRS) at 5-35 $\mu \mathrm{m}$ (Chen et al. 2006; Lisse et al. 2012), and VLTI/MIDI at 10-13 $\mu \mathrm{m}$ (Smith et al. 2009). As can be seen in Figure 1, the KIN spectrum across the $N$ band has adequate signal-to-noise ratio to resolve the $10 \mu \mathrm{m}$ silicate feature, which can be used to infer dust properties. The observed KIN excess also shows variability versus baseline length (Table 5). The outer disk in this object has been directly imaged (Wyatt et al. 2005; Matthews et al. 2010; Duchêne et al. 2014). Thus, instead of taking the disk inclination and P.A. as free parameters (zodi-level estimates presented in Table 2), we may assume that the exozodiacal disk has the same orientation. Using the values derived from the Duchêne et al. (2014) Herschel observations $\left(i_{\text {disk }}=47^{\circ}\right.$ with P.A. $\left.{ }_{\text {disk }}=117^{\circ}\right)$, we find a zodi level of $1870 \pm 211$ solar zodis.

\subsection{2. $\beta U M a$}

While $\beta$ Uma was resolved previously at $11.2 \mu \mathrm{m}$ (Moerchen et al. 2010), no significant spectral excess has been reported at $8-9 \mu \mathrm{m}$ so far, and the $\mathrm{KIN} \simeq 1 \%$ excess detection is then unprecedented in this wavelength range. Excesses are consistently detected at longer wavelengths with Spitzer/MIPS at 24 and $70 \mu \mathrm{m}$ and with Spitzer/IRS between 30 and $34 \mu \mathrm{m}$ (Chen et al. 2006). The circumstellar emission is also resolved by Herschel/PACS at 70 and $100 \mu \mathrm{m}$ (Booth et al. 2013), with a very clean fit to the data obtained by a narrow dust ring at about $43 \mathrm{AU}$ and seen close to edge-on $\left(i \simeq 84^{\circ}\right)$ with a P.A. of $114^{\circ}$. The KIN measurements are taken almost exactly $90^{\circ}$ away from the plane of the disk, with two scans obtained at a baseline azimuth of $\simeq 20^{\circ}$. They are then resolving the smaller apparent dimension of the inner zodiacal disk and could hence trace much colder/distant emission that the 200 mas FOV suggests. Assuming co-alignment with the outer ring, the derived zodi level is $390 \pm 96$ solar zodis.

\subsection{3. $\beta$ Leo}

Stock et al. (2010) combined nulling interferometry at $10 \mu \mathrm{m}$ using the MMT, KIN preliminary reduction results, and photometry from 3 to $100 \mu \mathrm{m}$ using Spitzer to study the debris disk around $\beta$ Leo over a broad range of spatial scales, corresponding to radii of $0.1-100 \mathrm{AU}$. They derived a complex debris system with relatively little material within $1 \mathrm{AU}$, an inner component with a color temperature of $600 \mathrm{~K}$ fitted by a dusty ring extending from about 2 to $3 \mathrm{AU}$, and a second component with a color temperature of $120 \mathrm{~K}$ fitted by a broad dusty emission zone extending from about 5 to $55 \mathrm{AU}$. Herschel 100 and $160 \mu \mathrm{m}$ images confirm this picture of the outer component, deriving a $40 \mathrm{AU}$ disk radius seen close to pole-on, together with a characteristic grain size smaller than $16 \mu \mathrm{m}$ (Matthews et al. 2010). An even more complex model is proposed by Churcher et al. (2011), with inner hot dust at $2 \mathrm{AU}$, an intermediate warm dust belt at $9 \mathrm{AU}$, and a colder component extending from 30 to 70 AU. Given the IWA and OWA of the KIN, the detected MIR excess must reside between 0.07 and 2.2 AU (Table 1). This is consistent with the presence of an inner ring around $2 \mathrm{AU}$, although we have no constraint on the excess spatial distribution inside of that radius. Finally, the ratio (1.041) measured between the semi-major and semi-minor disk radii of the Spitzer MIPS images (Stock et al. 2010) suggests that the disk is viewed at $20^{\circ} \pm 10^{\circ}$, close to face-on, which is consistent with the measured stellar inclination of 21.5 (Akeson et al. 2009). Adopting this inclination value for the inner component detected by the KIN, we derive a zodi level of $301 \pm 75$.

$$
\text { 5.1.4. } \zeta \text { Lep }
$$

$\zeta$ Lep has long been known for its $12 \mu \mathrm{m}$ IRAS excess indicative of dust material at unusually high temperature in the immediate vicinity of the star (Aumann \& Probst 1991). Infrared excesses have also been reported around this star at 24 and $70 \mu \mathrm{m}$ by Spitzer/MIPS yielding a [24]-[70] dust color temperature of $206 \mathrm{~K}$ (Su et al. 2006), fairly close to the $191 \mathrm{~K} \pm$ $3 \mathrm{~K}$ derived from a single blackbody fit to the Spitzer IRS spectrum measured between 5.5 and $35 \mu \mathrm{m}$ (Chen et al. 2006). The conversion of these spectral energy distribution (SED) derived apparent dust temperatures to actual dust location is, however, difficult without a proper knowledge of the grain basic properties, such as composition and size distribution. This was recently evidenced by Herschel spatially resolved observations of debris disks, showing dust location at significantly (up to 2.5 times) larger distance than predicted by simple blackbody fits to their SEDs (Booth et al. 2013). Prior attempts to directly resolve the warm disk component around $\zeta$ Lep have placed the warm dust component as close as $3 \mathrm{AU}$ from the star, using T-ReCS Gemini South observations at 10.4 and $18.3 \mu \mathrm{m}$ (Moerchen et al. 2007), and most likely $\leqslant 6$ AU based on early Keck images obtained at 11.7 and $17.9 \mu \mathrm{m}$ (Chen \& Jura 2001). Our resolved KIN observations are quite revealing in that respect, as they only show a $\simeq 1 \%$ leak excess, i.e., much smaller than the $13.6 \%$ mean IRS excess detected between 8.5 and $13 \mu \mathrm{m}$ (Chen et al. 2006), which has a typical rms uncertainty of $\simeq 1 \%$ in that wavelength range (Lawler et al. 2009). Even if the IRS excess is weaker (around 5\%-10\%) at the short $(8-10 \mu \mathrm{m})$ wavelength end that the KIN is mostly sensitive to, these two measurements are very difficult to reconcile. While we cannot formally rule out a substantial emission arising from within the $0.15 \mathrm{AU}$ KIN IWA, the most likely explanation is that most of the IRS-N band excess flux actually comes from regions outside of the KIN $\simeq 4$ AU FOV. Such a location would also be in line with recent two-belt modeling of warm debris disks' IRS spectra by Ballering et al. (2014), which used emission features to derive additional information about the grain properties and found a best-fit inner belt location of 5-6 AU in the case of $\zeta$ Lep (significantly larger than the $\simeq 3$ AU previously suggested). Assuming a disk inclination of $30^{\circ}$ with a P.A. of $50^{\circ}$, consistent with the Gemini South resolved observations of Moerchen et al. (2010), we find a zodi level of $243 \pm 73$ solar zodis.

$$
\text { 5.1.5. } \gamma \mathrm{Oph}
$$

This star was previously known to have excess starting at $\simeq 15 \mu \mathrm{m}$, and growing much larger at longer wavelengths (Spitzer IRS spectrum analysis; Chen et al. 2006; Su et al. 2008). It was listed as a possible KIN excess source in Paper I, and this new analysis confirms the presence of a small MIR excess around $10 \mu \mathrm{m}$. It is noteworthy that while the FIR excess emission is known to be larger around $\gamma$ Oph than around $\eta \mathrm{Crv}$ (Duchêne et al. 2014), the circumstellar excess is much weaker at $10 \mu \mathrm{m}$ around $\gamma$ Oph. This relative lack of inner warm dust 
is evidenced both by our KIN resolved measurements and by Spitzer IRS spectra, pointing to different spatial distributions around the two stars, with little dust within 5-10 AU in the case of $\gamma$ Oph. The Spitzer images ( $\mathrm{Su}$ et al. 2008) also spatially resolved the outer disk, suggesting a disk radius of $\simeq 520 \mathrm{AU}$ at $70 \mu \mathrm{m}$ and $\geqslant 260 \mathrm{AU}$ at $24 \mu \mathrm{m}$, yielding an inclination of $50^{\circ}$ and a P.A. of $55^{\circ}$. Assuming the same orientation for the exozodi disk, we derive a level of $290 \pm 94$ zodis.

\subsection{Stars with Likely or "Candidate" KIN Excesses}

As discussed in Section 4, the measured histograms of excess significance and zodi levels are strongly skewed toward higher detection levels. They show an excess of about 10-15 stars over what would be expected from random Gaussian measurement noise. Among them, a number of stars lie close to the KIN detection limit, at the $1 \sigma-3 \sigma$ confidence levels. All of these stars are reasonable candidates for a MIR excess and will be particularly interesting to observe with a higher-contrast instrument such as the LBTI. However, we concentrate here on KIN targets that have a broadband correlated excess significance larger than two, which probably really corresponds to a $\simeq 3 \sigma$ excess (Section 4.3). This selection criterion leaves the five bona fide KIN excess stars previously identified, plus three more A-type stars: Fomalhaut, Vega, and Altair. Noticeably, these three stars are hot and close enough that any putative dust at $300 \mathrm{~K}$ will extend significantly further than the KIN OWA (Table 1). Consequently, a significant fraction of the MIR circumstellar flux might have been missed around these stars.

\subsubsection{Fomalhaut}

While Fomalhaut does not show any significant excess in the $8-9 \mu \mathrm{m}$ bin, the measured excess leak increases at longer wavelengths, and the full $N$-band correlated excess is one of the highest measured in the overall sample. A separate analysis of Fomalhaut KIN observations (Mennesson et al. 2013), which included the survey results presented here and 2007 commissioning data, concluded the presence of a small excess leak with a mean value of $0.35 \% \pm 0.10 \%$ between 8 and $11 \mu \mathrm{m}$. This small KIN excess is likely the shortwavelength tail of the unresolved excess detected by Spitzer/ IRS at slightly longer MIR wavelengths (Stapelfeldt et al. 2004; $\mathrm{Su}$ et al. 2013), interpreted as the signature of a warm dust $(\simeq 170 \mathrm{~K})$ component located near the water frost line, inside of $\simeq 10 \mathrm{AU}$. A parametric modeling of the NIR (VLTI) and MIR (KIN) Fomalhaut interferometric data, complemented by spectrophotometric measurements from Spitzer and Herschel (Acke et al. 2012), concluded the existence of two distinct dust populations within a few AU (Mennesson et al. 2013; Lebreton et al. 2013): (1) a population of very small $(0.01-0.5 \mu \mathrm{m})$ and therefore unbound, hot $(1500 \mathrm{~K})$ dust grains confined in a narrow region $(\simeq 0.1-0.3 \mathrm{AU})$ at the sublimation rim of carbonaceous material; (2) a population of larger bound grains at about $2 \mathrm{AU}$ that is protected from sublimation and has a larger mass despite its fainter flux level. These previous analyses also suggested that the hot dust component may be produced by the release of small carbon grains following the disruption of dust aggregates originating from the warm component.

$$
\text { 5.2.2. Vega }
$$

Vega has been observed more than any other star in the KIN sample: a total of 18 scans were taken over five different epochs (from 2008 May to August), and nine different calibrators were used, all in an attempt to beat down the systematics. While these numerous observations yielded the smallest final null measurement uncertainties $\left(10^{-3}\right)$, excesses measured in the $8-9 \mu \mathrm{m}$ bin and broadband channels remain at the $\simeq 2 \sigma$ level in both cases. While a $10 \mu \mathrm{m}$ excess is likely (see Section 4.3), we prefer to consider Vega a "KIN excess candidate." It is obviously a prime target for further observations with the LBTI nuller, as only a minor gain in accuracy, or a larger FOV, could either confirm or rule out this candidate excess. We also note that owing to its limited FOV ( $\simeq 1.5 \mathrm{AU}$ radius at Vega's distance), the KIN may have missed a large fraction of dust emission at $300 \mathrm{~K}$ or lower (Table 1). Since the Vega system is seen nearly pole-on (Monnier et al. 2012), the KIN observations are in particular completely insensitive to dust at $170 \mathrm{~K}(\simeq 14 \mathrm{AU})$, the temperature derived from Spitzer/IRS spectrum analysis ( $\mathrm{Su}$ et al. 2013). In any case, the KIN leak excess $(3 \sigma)$ upper limit is about $0.5 \%$, which translates into a maximum physical excess of about $1 \%$ within the instrument FOV. ${ }^{20}$ Once again, this result is quite surprising given the $\simeq 1 \%$ NIR excesses previously reported around Vega by various interferometers (CHARA: Absil et al. 2006; IOTA: Defrere et al. 2011). Assuming no temporal variability of the excess between the NIR CHARA/ IOTA and MIR KIN measurements, it would have to originate in very hot dust grains located close to the star, yet small enough to remain largely undetected at $10 \mu \mathrm{m}$ (Mennesson et al. 2011).

\subsubsection{Altair}

Altair has no excess reported at either 24 or $70 \mu \mathrm{m}$ (Gaspar et al. 2013). A solidly significant MIR KIN excess would then be particularly interesting as it would be the only one detected around a star with no prior evidence of an outer cold dust reservoir. It is noteworthy that out of the 25 stars with data presented in Paper I, only Altair shows significantly different results in this new reduction (Table 2). While Paper I reported a marginal excess between 8 and $9 \mu \mathrm{m}$, we find no evidence for it here. The reason is that a single calibrator (HD 184406) was used for Altair null measurements presented in Paper I, and its diameter was apparently wrongly estimated from the FATCAT K I measurements, causing an overestimation of Altair's calibrated null depth. Indeed, Paper I used a uniform disk (UD) diameter of $3.05 \pm 0.07$ mas for HD 184406, while we use here the smaller UD diameter of $2.25 \pm 0.06$ mas measured in 2013 October by the CHARA interferometer (N. Scott, 2013, private communication). Note that this new value is in remarkable agreement with the $K$-band UD diameter of $2.24 \pm 0.02$ mas estimated from precise SED fitting of interferometric calibrators (Bordé et al. 2002). The revised calibrator diameter value strongly reduces the excess leak measured at short wavelengths $(8-9 \mu \mathrm{m}$ bin), but this calibration effect is less at longer wavelengths, and we still find a rather large excess significance (2.55) for Altair's broadband $8-13 \mu \mathrm{m}$ excess. The excess spectral shape is also reminiscent of that found around Fomalhaut, steadily increasing with wavelength. Another difficulty in assessing a possible excess around Altair is that the star is large enough that uncertainties on its angular diameter can result in significant errors on the excess leaks derived. Altair's photosphere is known to be elongated owing to fast rotation (van Belle et al. 2001). Even though we took this effect into account and adopted the diameter measured at $H$ band by the CHARA MIRC instrument

\footnotetext{
20 The exact scaling factor between the two excess quantities depends on the spatial distribution of the excess, but over a wide range of possible models (e.g., thin annular ring or uniform emission inside the KIN FOV), the conversion factor is about two.
} 
(Monnier et al. 2007) over the range of P.A.s sampled by our observations (3.64 mas \pm 0.03 mas), we recognize that the $N$ band diameter may be significantly different, especially in the presence of strong gravity-induced LD. For this reason, and although the existence of a small MIR excess is possible, there might be some residual calibration issues for Altair, and we also prefer to classify it as a "KIN excess candidate."

\section{CONCLUSIONS}

A total of 47 stars have been surveyed by the KIN between 2008 and 2011, including 40 stars with no known companion within the instrument FOV. The overall data have been reduced in a common manner, allowing an assessment of the amount of resolved off-axis emission above the stellar photosphere as a function of wavelength between 8 and $13 \mu \mathrm{m}$. Only five stars show an 8-9 $\mu \mathrm{m}$ MIR excess ( $\eta \mathrm{Crv}, \beta$ Leo, $\beta$ UMa, $\zeta$ Lep, and $\gamma \mathrm{Oph}$ ), while a marginal $N$-band excess is also detected around Fomalhaut. While the results' statistical significance is necessarily limited by the sample size, we only detect MIR excesses around main-sequence stars with types earlier than F2.

We find that all stars with a bona fide or candidate KIN excess had a previously detected FIR excess. A statistical analysis of the whole data set shows a more general correlation between the level of (zodi-like) warm dust emission measured at 8-9 $\mu \mathrm{m}$ and the presence of (Kuiper-like) cold dust. While warm dust detected by the KIN necessarily resides within the inner few AU, it thus likely finds its origin in the outer regions of the system rather than through in situ collisions of large parent bodies. We also found that stars with previously known cold FIR excesses generally have zodi levels close to the KIN detection limit of a few hundred zodis $(1 \sigma)$. This observation is broadly consistent with a scenario where an outer planetesimal belt is feeding dust to the inner system through the balanced effects of P-R drag and grain collisions, which predicts that the level of warm dust emission is fairly insensitive to the properties of the outer disk, as long as it is bright enough. These cold excess stars constitute prime science targets for higher precision/larger FOV MIR observations of exozodiacal dust, but they are probably bad targets for future missions designed to directly image Earth analogs.

We also find that stars with NIR excesses recently reported by interferometry do not show any corresponding excesses detectable by the KIN. This lack of correlation could either point to a different mechanism for the generation of the NIR circumstellar emission or call for large amounts of very hot grains piling up close to the sublimation radius of these stars, small enough to have a low MIR emissivity and remain undetected by the KIN.

Finally, assuming that the 20 solar-type stars with no previously detected IR excess observed by the KIN constitute an independent and identically distributed sample, and testing a wide range of reasonable shapes for the underlying distribution, we find a most likely median zodi level of 20 zodis or less for these stars. Assuming a lognormal zodi luminosity function, we further established with high confidence (95\%) that at least $50 \%$ of such stars have zodi levels lower than 60 zodis. This is potentially good news for a future direct exo-Earth imaging mission, although an exozodiacal cloud at 60 times the solar level is still problematic. MIR observations of a larger sample with lower detection limits are then still desirable for the proper design of such missions and optimum target selection.

The Keck Interferometer was funded by the National Aeronautics and Space Administration (NASA). Part of this work was performed at the Jet Propulsion Laboratory, California Institute of Technology, and at the NASA Exoplanet Science Center (NExScI), under contract with NASA. The Keck Observatory was made possible through the generous financial support of the W. M. Keck Foundation. This research has made use of the Washington Double Star Catalog maintained at the U.S. Naval Observatory. M.C.W. is grateful for support from the European Union through ERC grant number 279973.

\section{APPENDIX A \\ KIN OBSERVABLE}

Four beams are recombined by the KIN system (Colavita et al. 2009). A split mirror located downstream of each Keck Telescope adaptive optics system-close to a pupil planedivides the light gathered by each telescope into "left" and "right" beams. Long-baseline $(85 \mathrm{~m})$ interferometric recombination first occurs separately between the two left beams and between the two right beams, with each beam pair either recombined constructively (at peak) or destructively (at null). The resulting left and right output fields are then coherently recombined using a standard Michelson interferometer, called the "cross-combiner." As the optical delay is rapidly scanned inside the cross-combiner, one measures a fringe amplitude with left and right beam pairs at null and then with left and right beam pairs at peak. The null depth is defined as the ratio of the crosscombiner fringe amplitudes obtained at null and at peak and is measured in each of 10 independent spectral channels covering the full $N$ band $(8-13 \mu \mathrm{m})$. The rationale for this complex fourbeam combination and modulation is that the resulting measured null depth is free of slow drifts in the incoherent background (Mennesson et al. 2005; Serabyn et al. 2012), a source of strong potential bias for ground-based interferometric observations in the thermal IR. Two different scales and baselines are then at play: the interferometric nulling baseline of length $B \simeq 85 \mathrm{~m}$, separating the telescope centers, and the short cross-combiner baseline $b \simeq 4 \mathrm{~m}$, characteristic of the interference between the "left" and "right" parts of a given Keck Telescope.

For a perfectly calibrated instrument, defined as providing a null depth of zero on a point source, the measured monochromatic astrophysical null $N_{\text {ast }}$ can be related to the source brightness distribution on the sky $I(\boldsymbol{\theta})$ via (Serabyn et al. 2012; Mennesson et al. 2013)

$$
N_{\mathrm{ast}}(\lambda)=\frac{\int I(\boldsymbol{\theta}) \sin ^{2}(\pi \boldsymbol{B} \cdot \boldsymbol{\theta} / \lambda) \cos (2 \pi \boldsymbol{b} \cdot \boldsymbol{\theta} / \lambda) \sqrt{T_{L}(\boldsymbol{\theta}) T_{R}(\boldsymbol{\theta})} d \boldsymbol{\theta}}{\int I(\boldsymbol{\theta}) \cos ^{2}(\pi \boldsymbol{B} \cdot \boldsymbol{\theta} / \lambda) \cos (2 \pi \boldsymbol{b} \cdot \boldsymbol{\theta} / \lambda) \sqrt{T_{L}(\boldsymbol{\theta}) T_{R}(\boldsymbol{\theta})} d \boldsymbol{\theta}},
$$

where $T_{L}(\boldsymbol{\theta})$ and $T_{R}(\boldsymbol{\theta})$ designate the sky transmission of the left and right Keck beams, respectively. These are computed from the telescope's orientation and from the overall beam train propagation, which includes an intermediary focal plane pinhole limiting the geometric FOV radius to 300 mas. As shown in Equation (A1), in the case of an extended source, the measured null level is affected not only by the long-baseline nulling pattern (fast oscillating squared sine term) but also by the cross fringe pattern (slowly oscillating cosine term) and by the lobe antenna of each single beam. The overall transmission pattern of the KIN is illustrated in Figure 11, showing the case of Fomalhaut observations at the time of meridian transit. Taking into account the individual beam transmission (finite FOV) and cross-combiner modulation effects (small baseline $b$ ), the source brightness distribution effectively seen by the KIN can 


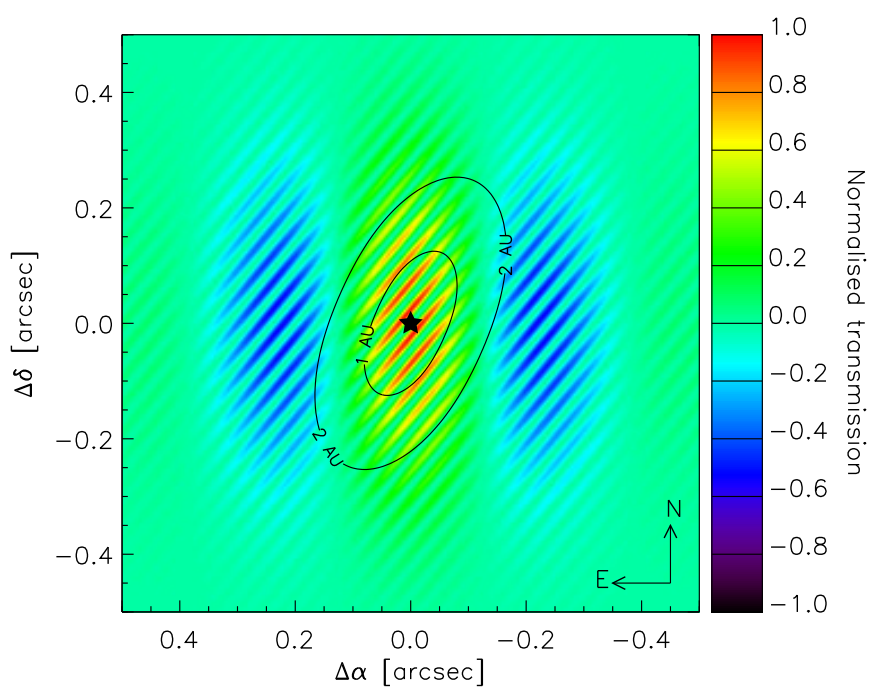

Figure 11. Keck Nuller sky transmission at $10 \mu \mathrm{m}$, when observing Fomalhaut at meridian transit on 2008 July 17 (Julian date: 2,454,664.07; projected baseline: $67.8 \mathrm{~m}$; azimuth: 49.6). North is up, east is to the left. High-frequency fringes correspond to the long baseline separating the telescopes. The low-frequency modulation is produced by interference between the subapertures of a single Keck Telescope ("cross-combiner" fringes); these fringes are aligned with the east-west direction when observing a star at transit. The contours indicate inner regions of the Fomalhaut system $\left(i=66^{\circ}\right.$, P.A. $\left.=156^{\circ}\right)$, showing that the KIN is sensitive to dust emission in the 0.05-2 AU range at the star distance. As noted before (Mennesson et al. 2013), a curious effect of the KIN four-beam combination is that for emission sources extending further out than $\lambda / b$ in the direction of the cross-combiner baseline $b(\simeq 4 \mathrm{~m})$, some regions will contribute a "negative leakage," i.e., effectively decrease the observed null depth. This is illustrated by the two blue areas of negative transmission centered around \pm 0 !' 25 in R.A.

(A color version of this figure is available in the online journal.)

be written as

$$
I_{\mathrm{KIN}}(\boldsymbol{\theta})=I(\boldsymbol{\theta}) \cdot \sqrt{T_{L}(\boldsymbol{\theta}) T_{R}(\boldsymbol{\theta})} \cdot \cos (2 \pi \boldsymbol{b} \cdot \boldsymbol{\theta} / \lambda) .
$$

The corresponding interferometric visibility measured at baseline $\boldsymbol{B}$ is defined by

$$
V_{B}=\frac{\int I_{\mathrm{KIN}}(\boldsymbol{\theta}) \cdot e^{(j 2 \pi \boldsymbol{B} \cdot \boldsymbol{\theta} / \lambda)} d \boldsymbol{\theta}}{\int I_{\mathrm{KIN}}(\boldsymbol{\theta}) \cdot d \boldsymbol{\theta}}
$$

Replacing in Equation (A1), one finally obtains after a little algebra

$$
N_{\text {ast }}(\lambda)=\frac{\left(1-\left|V_{B}\right|\right)}{\left(1+\left|V_{B}\right|\right)} .
$$

This is the usual relation between astronomical null depth and visibility, except that the null or visibility is measured here for a "modified" source brightness distribution, which incorporates both the KIN instrument-limited FOV and the short-baseline cosine modulation (Equation (A2)).

\section{APPENDIX B}

\section{CONFIDENCE INTERVALS FOR PARAMETERS ESTIMATED BY MAXIMUM LIKELIHOOD METHOD}

Given the maximum likelihood function $L$ defined in Section 4.6.2 and the maximum likelihood parameters $\hat{\boldsymbol{\theta}}=$ $\left(\hat{\theta_{1}}, \hat{\theta_{2}}, \ldots, \hat{\theta_{p}}\right)$, we define the profile likelihood function for a single parameter of interest, e.g., $\theta_{1}$, as (Venzon \& Moolgavkar 1988)

$$
R\left(\theta_{1}\right)=\frac{\max _{\left(\theta_{2}, \ldots, \theta_{p}\right)} L\left(\theta_{1}, \theta_{2}, \ldots, \theta_{p}\right)}{L(\hat{\boldsymbol{\theta}})},
$$

where the numerator is simply, for each value of parameter $\theta_{1}$, the maximum of the likelihood function over the remaining parameters (if any). For a large enough sample (asymptotic normal assumption), the quantity $X^{2}=-2 \ln R\left(\theta_{1}\right)$ follows a chi-square distribution with 1 degree of freedom (Venzon \& Moolgavkar 1988), meaning that $X$ follows a unit normal distribution. This property allows us to compute confidence intervals for each of the derived maximum likelihood parameters, which is much preferable to giving $1 \sigma$ uncertainties with no confidence levels attached to them. A $100(1-\alpha) \%$ likelihood-based confidence interval for $\theta_{1}$ is obtained for the set of $\theta_{1}$ values verifying

$$
R\left(\theta_{1}\right) \geqslant \exp \left(-\chi_{(1-\alpha ; 1)}^{2} / 2\right) .
$$

For example, a (two-sided) $90 \%$ confidence interval $(95 \%$ on upper or lower bounds) corresponds to a $\chi^{2}$ value of 2.706 , and the condition is then that $R\left(\theta_{1}\right)$ be higher than 0.259 . This method is used to compute confidence intervals for the maximum likelihood parameters derived in Section 4.6.2 and for the (profile) likelihood curves shown in Figures 6-8.

Another possible approach, which does not rely on the asymptotic normal assumption, is to compute Bayesian confidence bounds for a given parameter by integrating its marginal distribution given the data. In the case of a lognormal distribution with parameters $(\mu, \sigma)$, the confidence level for a given upper limit $\mu^{u}$ on parameter $\mu$ is given by

$$
P\left(\mu \leqslant \mu^{u}\right)=\frac{\int_{-\infty}^{\mu^{u}} \int_{0}^{\infty}(L(\mu, \sigma) / \sigma) d \sigma d \mu}{\int_{-\infty}^{\infty} \int_{0}^{\infty}(L(\mu, \sigma) / \sigma) d \sigma d \mu} .
$$

As an independent check, we applied this Bayesian approach to the likelihood function $L$ computed for the ensemble of solartype stars with no excess previously detected (Group 1), assuming a lognormal zodi distribution. Using the above equation to compute confidence levels for the distribution $\mu$ parameter, we found values very similar to those presented in Section 4.6.4: the ensemble median zodi level $(\exp (\mu))$ upper limit is 55 solar zodis at $95 \%$ confidence and 90 zodis at $99 \%$ confidence.

\section{REFERENCES}

Absil, O., Defrère, D., Coudé du Foresto, V., et al. 2013, A\&A, 555, 104 Absil, O., di Folco, E., Mérand, A., et al. 2006, A\&A, 452, 237

Absil, O., Le Bouquin, J.-B., Berger, J.-P., et al. 2011, A\&A, 535, 68 Absil, O., Mennesson, B., Le Bouquin, J.-B., et al. 2009, ApJ, 704, 150 Acke, B., Min, M., Dominik, C., et al. 2012, A\&A, 540, 125

Akeson, R. L., Ciardi, D. R., Millan-Gabet, R., et al. 2009, ApJ, 691, 1896 Aufdenberg, J. P., Mérand, A., Coudé du Foresto, V., et al. 2006, ApJ, 651,617

Aumann, H. H., \& Probst, R. G. 1991, ApJ, 368, 264

Backman, D., Marengo, M., Stapelfeldt, K., et al. 2009, ApJ, 690, 1522 Ballering, N., Rieke, G. H., \& Gaspar, A. 2014, ApJ, 793, 57

Beichman, C. A., Bryden, G., Gautier, T. N., et al. 2005, ApJ, 626, 1061 Beichman, C. A., Bryden, G., Stapelfeldt, K. R., et al. 2006a, ApJ, 652, 1674 Beichman, C. A., Tanner, A., Bryden, G., et al. 2006b, ApJ, 639, 1166 Beust, H., \& Valiron, P. 2007, A\&A, 466, 201

Boden, A. F., Koresko, C. D., van Belle, G. T., et al. 1999, ApJ, 515, 356 Bonneau, D., Clausse, J.-M., Delfosse, X., et al. 2006, A\&A, 456, 789 Bonsor, A., Augereau, J.-C., \& Thebault, P. 2012, A\&A, 548, 104

Bonsor, A., Kennedy, G. M., Crepp, J. R., et al. 2013, MNRAS, 431, 3025 Bonsor, A., Raymond, S. N., Augereau, J.-C., \& Ormel, C. W. 2014, MNRAS, 441,2380

Bonsor, A., \& Wyatt, M. C. 2012, MNRAS, 420, 299

Booth, M., Kennedy, G., Sibthorpe, B., et al. 2013, MNRAS, 428, 1263

Bordé, P., Coudé du Foresto, V., Chagnon, G., \& Perrin, G. 2002, A\&A, 393, 183

Bryden, G., Beichman, C. A., Carpenter, J. M., et al. 2009, ApJ, 705, 1226 Burgasser, A., Kirkpatrick, J. D., Reid, I. N., et al. 2003, ApJ, 586, 512 
Chen, C. H., \& Jura, M. 2001, ApJL, 560, L171

Chen, C. H., Sargent, B. A., Bohac, C., et al. 2006, ApJS, 166, 351

Churcher, L. J., Wyatt, M. C., Duchêne, G., et al. 2011, MNRAS, 417, 1715

Cohen, M., Walker, R. G., Carter, B., et al. 1999, AJ, 117, 1864

Colavita, M. M., Serabyn, E., Millan-Gabet, R., et al. 2009, PASP, 121, 1120

Colavita, M. M., Wizinowich, P. L., Akeson, R. L., et al. 2013, PASP, 125, 1226

Defrere, D., Absil, O., Augereau, J.-C., et al. 2011, A\&A, 534, 5

Di Benedetto, G. P. 2005, MNRAS, 357, 174

Dodson-Robinson, S. E, Beichman, C. A., Carpenter, J. M., \& Bryden, G. 2011, AJ, 141, 11

Duchêne, G., Arriaga, P., Wyatt, M., et al. 2014, ApJ, 784, 148

Eiroa, C., Marshall, J. P., Mora, A., et al. 2013, A\&A, 555, 11

Fischer, D., Marcy, G. W., \& Spronck, J. F. P. 2014, ApJS, 210, 5

Fujiwara, H., Onaka, T., Takita, S., et al. 2012, ApJ, 759, 18

Gaspar, A., Rieke, G., \& Zoltan, B. 2013, ApJ, 768, 25

Gezari, D. Y., Schmitz, M., Pitts, P. S., \& Mead, J. M. 1993, Far Infrared Supplement: Catalog of Infrared Observations (3rd ed.; NASA Ref. Pub. 1294; Washington, DC: NASA)

Gomes, R., Levison, H. F., Tsiganis, K., \& Morbidelli, A. 2005, Natur, 435,466

Habing, H. J., Dominik, C., Jourdain de Muizon, M., et al. 2001, A\&A, 365,545

Hale, A. 1994, AJ, 107, 306

Hartkopf, W. I., Mason, B. D., \& Morley, C. E. 2001, Sixth Calaog of Orbits of Visual Binary Stars (Washington, DC: US Naval Observatory) (http://ad.usno.navy.mil/wds/orb6/orb6text.html)

Kelsall, T., Weiland, J. L., Franz, B. A., et al. 1998, ApJ, 508, 44

Kennedy, G. M., \& Wyatt, M. C. 2013, MNRAS, 433, 233

Köenig, B., Fuhrmann, K., Neuhuser, R., Charbonneau, D., \& Jayawardhana, R. 2002, A\&A, 394, 43

Lagrange, A.-M., Gratadour, D., Chauvin, G., et al. 2009, A\&A, 493L, 21

Lawler, S. M., Beichman, C. A., Bryden, G., et al. 2009, ApJ, 705, 89

Lebreton, J., van Lieshout, R., Augereau, J.-C., et al. 2013, A\&A, 555, 146

Lisse, C. M., Wyatt, M. C., Chen, C. H., et al. 2012, ApJ, 747, 93

Macintosh, B., Graham, J. R., Ingraham, P., et al. 2014, PNAS, 111, 12661

Mason, B. D., Wycoff, G. L., Hartkopf, W. I., Douglass, G. G., \& Worley, C. E. 2001, AJ, 122, 3466

Matthews, B. C., Sibthorpe, B., Kennedy, G., et al. 2010, A\&A, 518L, 135

Mennesson, B., Absil, O., Lebreton, J., et al. 2013, ApJ, 763, 119

Mennesson, B., Leger, A., \& Ollivier, M. 2005, Icar, 178, 570
Mennesson, B., Serabyn, E., Hanot, C., et al. 2011, ApJ, 736, 14

Merand, A., Bordé, P., \& Coudé du Foresto, V. 2006, A\&A, 447, 783

Millan-Gabet, R., Serabyn, E., Mennesson, B., et al. 2011, ApJ, 734, 67

Moerchen, M. M., Telesco, C. M., \& Packham, C. 2010, ApJ, 723, 1418

Moerchen, M. M., Telesco, C. M., Packham, C., \& Kehoe, T. J. J. 2007, ApJL, 655, L109

Monnier, J. D., Zhao, M., Pedretti, E., et al. 2007, Sci, 317, 342

Monnier, J. D., Che, X., Zhao, M., et al. 2012, ApJ, 761, 3

Moro-Martin, A., Wolf, S., Malhotra, R., et al. 2005, ApJ, 621, 1079

Nesvorny, D., Jenniskens, P., Levison, H. F., et al. 2010, ApJ, 713, 816

Neugebauer, G., \& Leighton, R. B. 1969, Two-Micron Sky Survey: A Preliminary Catalog (SP-3047; Washington, DC: NASA)

Rhee, J. H., Song, I., Zuckerman, B., \& McElwain, M. 2007, ApJ, 660, 1556

Rieke, G. H., Su, K. Y. L., Stansberry, J. A., et al. 2005, ApJ, 620, 1010

Roberts, L. C., Jr., Turner, N. H., ten Brummelaar, T. A., Mason, B. D., \& Hartkopf, W. I. 2011, AJ, 142, 175

Schmelling, M. 1995, PhyS, 51, 676

Serabyn, G., Mennesson, B., Colavita, M. M., Koresko, C., \& Kuchner, M. J. 2012, ApJ, 748, 55

Sheret, I., Dent, W. R. F., \& Wyatt, M. C 2004, MNRAS, 348, 1282

Smith, R., Wyatt, M. C., \& Haniff, C. A. 2009, A\&A, 503, 265

Stapelfeldt, K. R., Holmes, E. K., Chen, C., et al. 2004, ApJS, 154, 458

Stock, N. D., Su, K. Y. L., Liu, W., et al. 2010, ApJ, 724, 1238

Su, K. Y. L., Rieke, G. H., Malhotra, R., et al. 2013, ApJ, 763, 118

Su, K. Y. L., Rieke, G. H., Stansberry, J. A., et al. 2006, ApJ, 653, 675

Su, K. Y. L., Rieke, G. H., Stapelfeldt, K. R., et al. 2008, ApJL, 679, L125

Trilling, D. E., Bryden, G., Beichman, C. A., et al. 2008, ApJ, 674, 1086

Tuomi, M., Jones, H. R. A., Jenkins, J. S., et al. 2013, A\&A, 551, 79

van Belle, G. T., Ciardi, D. R., Thompson, R. R., Akeson, R. L., \& Lada, E. A. 2001, ApJ, 559, 1155

van Lieshout, R., Dominik, C., Kama, M., \& Min, M. 2014, A\&A, 571, 51

Venzon, D. J., \& Moolgavkar, S. H. 1988, JApS, 37, 87

Weinberger, A. J., Becklin, E. E., Song, I., \& Zuckerman, B. 2011, ApJ, 726,72

Weinberger, A. J., Wyatt, M. C., Bryden, G., et al. 2014, ApJ, submitted

Wyatt, M. C. 2005, A\&A, 433, 1007

Wyatt, M. C., Greaves, J. S., Dent, W. R. F., \& Coulson, I. M. 2005, ApJ, 620,492

Wyatt, M. C., Kennedy, G., Sibthorpe, B., et al. 2012, MNRAS, 424, 1206

Wyatt, M. C., Smith, R., Greaves, J. S., et al. 2007, ApJ, 658, 569 